\title{
1 The complex evolution of the metazoan HSP70 gene family
}

3 Er-meng Yu ${ }^{1,2}$, Tatsuki Yoshinaga ${ }^{3}$, Frank L. Jalufka ${ }^{1}$, Hashimul Ehsan ${ }^{1}$, David B. Mark Welch ${ }^{*}$,

4 Gen Kaneko $^{1 *}$

5

$6 \quad{ }^{1}$ School of Arts \& Sciences, University of Houston-Victoria, Victoria, TX.

$7 \quad{ }^{2}$ Key Laboratory of Tropical \& Subtropical Fishery Resource Application \& Cultivation, Key

8 Laboratory of Aquatic Animal Immune Technology of Guangdong Province, Pearl River Fisheries

9 Research Institute of CAFS, Guangzhou, China.

$10{ }^{3}$ School of Marine Biosciences, Kitasato University, Kanagawa, Japan.

$11{ }^{4}$ Josephine Bay Paul Center for Comparative Molecular Biology and Evolution, Marine Biological 12 Laboratory, MA.

13

\section{4 *Corresponding author:}

15 Gen Kaneko, kanekog@uhv.edu

16 David Mark Welch, dmarkwelch@mbl.edu

17 


\section{Abstract}

19 The metazoan 70-kDa heat shock protein (HSP70) family contains several members localized in

20 different subcellular compartments. The cytosolic members have been classified into inducible

21 HSP70s and constitutive heat shock cognates (HSC70s), but their distinction and evolutionary

22 relationship remain unclear because of occasional reports of "constitutive HSP70s" and the lack of

23 cross-phylum comparisons. Here we provide novel insights into the evolution of these important

24 molecular chaperones. Phylogenetic analyses of $\sim 100$ full-length HSP70s revealed an ancient

25 duplication that gave rise to two lineages from which all metazoan cytosolic HSP70s descend. One

26 lineage (A) contains a relatively small number of Lophotrochozoan and Ecdysozoan genes, none of

27 which have been shown to be constitutively expressed (i.e., either inducible or unknown). The other

28 lineage (B) included both inducible and constitutive genes from diverse phyla. Species-specific

29 duplications are present in both lineages, and Lineage B contains well-supported phylum-specific

30 clades for Rotifera, Nematoda, and Chordata. Some genes in Lineage B have likely independently

31 acquired inducibility, which may explain the sporadic distribution of "HSP70" or "HSC70" in

32 previous analyses. Consistent with the diversification history within each group, inducible members

33 show lower purifying selection pressure compared to constitutive members. These results illustrate

34 the evolutionary history of the HSP70 family, encouraging us to propose a new nomenclature:

35 "HSP70 + subcellular localization + linage + copy number in the organism + inducible or

36 constitutive, if known.” e.g., HSP70cA1i for cytosolic Lineage A, copy 1, inducible.

38 Key words: heat shock cognates, molecular evolution, rotifer, stress inducibility, synonymous and 39 nonsynonymous substitution. 


\section{Introduction}

42 The 70-kDa heat shock protein (HSP70) family members play important roles in various

43 cellular processes including heat shock response, folding of newly synthesized proteins, protein

44 transport, and protein degradation. These apparently diverse functions are attributed to their

45 chaperone activity (Daugaard et al. 2007; Hartl et al. 2011), by which they prevent the aggregation

46 and misfolding of target proteins. Typically, HSP70 family members bind to denaturing or newly

47 synthesized proteins by recognizing up to ten hydrophobic amino acid residues exposed to the

48 protein surface because of the misfolding. The release of the HSP70 members, which is triggered by

49 ATP hydrolysis, facilitates the proper folding of the target proteins (Zuiderweg et al. 2017). The

50 HSP70 family contains organelle-specific members localized in cytosol, endoplasmic reticulum

51 (ER), mitochondria, and chloroplasts (Miernyk 1997), and these organelle-specific types not only

52 perform chaperoning functions in the organelles but are also known to contribute to protein

53 transport across organelle membranes.

54 HSP70 family members are often upregulated upon various stresses that disrupt protein

55 folding, such as heat treatment, exposure to toxic materials, ultraviolet irradiation, and pathogen

56 attack (Sørensen et al. 2003; Baird et al. 2006). The upregulation is primarily regulated at the

57 transcription level by transcription factors called heat shock factors (HSFs), particularly HSF1.

58 Under unstressed conditions, HSF1 remains in a monomeric state. Various stresses lead to the

59 formation of HSF1 trimers, which in turn bind to heat shock elements (HSEs) in the promoter

60 region of HSP70 family member genes and promote their transcription. The HSF1-dependent

61 transactivation system is generally conserved among eukaryotes, although species-specific

62 differences in HSF1 function have been reported in many organisms such as the fruit fly Drosophila

63 melanogaster (Jedlicka et al. 1997; Marchler and Wu 2001) and yeast Saccharomyces cerevisiae

64 (Sorger and Pelham 1988). The expression of HSP70 family member is also regulated by many

65 other mechanisms such as the unfolded protein response, chromatin modification, and other

66 transcription factors depending on the type of stress and subcellular localization (De Nadal et al. 
67 2011; Garbuz 2017). HSP70 family member genes have therefore been used as biomarkers of

68 environmental stresses both in laboratory and field experiments (Sanders 1993; Ceyhun et al. 2010;

69 Judge et al. 2011).

70 On the other hand, several cytosolic HSP70 family members show constitutive expression

71 patterns. These proteins are involved in the folding of newly synthesized proteins and are

72 traditionally called 70-kDa heat shock cognates (HSC70). However, the distinction between

73 HSP70s and HSC70s remains unclear because of the occasional reports of "constitutive HSP70s,"

74 especially from invertebrates (Jayasena et al. 1999; Piano et al. 2002; Liu et al. 2017). Stress-

75 induced upregulation of HSC70 has also been reported in several animals including shrimps (Luan

76 et al. 2010), snails (Zheng et al. 2012), and fish (Yabu et al. 2011). Furthermore, little is known for

77 the evolutionary relationship between metazoan HSP70s and HSC70s because of the lack of cross-

78 phylum comparisons. A previous phylogenetic analysis indicated that metazoan HSP70 family

79 members can be classified into invertebrate HSP70s, vertebrate HSP70s, and HSC70s from both

80 vertebrates and invertebrates (Kourtidis et al. 2006), but this study comprises only a few phyla. In-

81 depth phylogenetic analysis on HSP70 family members with a broad sampling would offer further

82 insight into the classification of this important group of molecular chaperones.

83 In this study, we sought to trace the evolutionary history of metazoan HSP70 family members

84 by cross-phylum phylogenetic analyses with a particular attention to their stress inducibility. The

85 specific hypothesis was that the cross-phylum analysis would provide the essential information to

86 solve the evolutionary relationship between metazoan HSP70s and HSC70s. In order to add insights

87 from an emerging model in evolutionary biology to the analyses, we first cloned two stress-

88 inducible HSP70 family member genes from a monogonont rotifer, Brachionus plicatilis. The

89 phylum Rotifera, composed of mostly microscopic aquatic animals with about 1,000 cells and a

90 ciliated head structure, is part of the Gnathifera, a group of basal-branching phyla related to

91 Lophotrochozoa, which contains Mollusca and Annelida. Together these groups form a sister-clade

92 to Ecdysozoa, which contains the established invertebrate models Caenorhabditis elegans and $D$. 
93 melanogaster (Struck et al. 2014; Fröbius and Funch 2017). Genetic information from Gnathifera

94 remains limited, making rotifers useful models in evolutionary studies. Our results added novel

95 groups of stress-inducible HSP70, providing important insight into the evolutionary history of the

96 metazoan HSP70 family.

97

$98 \quad$ Results

99 Molecular Characterization of Rotifer HSP70 Genes

100 A combination of 3' and 5' RACE identified two B. plicatilis HSP70 genes consisting of 2,121

101 and 2,248 bp, designated as HSP70cB1i and HSP70cB2i, respectively (see Discussion for the

102 nomenclature). The nucleotide sequence of the open reading frames, as well as the absence of

103 introns, were ascertained by single PCR experiments using cDNA or genomic DNA, respectively,

104 followed by DNA sequencing. The deduced amino acid sequences of HSP70-1 and HSP70-2 genes

105 were more than 99\% identical to each other (supplementary fig. S1). Both contain the HSP70

106 protein family signatures, IDLGTTYS, IFDLGGGTFDVSIL, and IVLVGGSTRIPKVQK (Prosite

107 motifs PS00297, PS00329, and PS01036, respectively), as well as the non-organelle stress protein

108 motif RARFEEL found in several cytosolic HSP70s (Lo et al. 2004; Cottin et al. 2008; Simoncelli

109 et al. 2010; Zheng et al. 2012). The major difference between B. plicatilis HSP70 genes was the

110 number of GGMP repeats, which are involved in binding to the HSP70-HSP90-organizing protein

111 (Hop), a cofactor of HSP70 (Demand et al. 1998). The nucleotide sequences of B. plicatilis

112 HSP70cB1i and HSP70cB2i genes were registered into the DDBJ/EMBL/GenBank databases with

113 accession numbers AB775784 and AB775785, respectively. The two HSP70 genes were also found

114 in the B. plicatilis genomic sequence.

115 We next examined whether the mRNA levels of B. plicatilis HSP70s are increased by heat

116 stress. Due to the high sequence identity between B. plicatilis HSP70 genes and difficulty in

117 designing a TaqMan probe, we employed semi-quantitative RT-PCR to measure their respective

118 mRNA levels. Primers were designed to amplify DNA fragments of 112 and 124 bp from 
119 HSP70cB1i and HSP70cB2i cDNAs, respectively (fig. 1A), and the PCR products were separated

120 using a polyacrylamide gel. The mRNA levels of HSP70cB1i and HSP70cB2i in heat-treated

121 rotifers were 2.9 and 7.5 times higher, respectively, than those in control rotifers (fig. 1B and 1C).

122 These results indicate that both genes encode heat-inducible HSP70.

123 Since the two B. plicatilis HSP70 genes showed a similar expression pattern in response to

124 heat stress, we used quantitative real-time PCR primers that amplify both HSP70cB1i and

125 HSP70cB2i genes in the subsequent time-course expression analyses (fig. 1D). The relative mRNA

126 levels were increased by heat stress at $40^{\circ} \mathrm{C}$ for $10 \mathrm{~min}$ and reached at the maximum level $2 \mathrm{~h}$ after

127 the heat stress. The difference in mRNA levels between the control and $2 \mathrm{~h}$ groups was statistically

128 significant. The mRNA levels of $B$. plicatilis HSP70s remained twofold $8 \mathrm{~h}$ after the heat stress. We

129 then performed in situ hybridization using DIG-labeled RNA probes (fig. 1E). The probe region

130 contained only five base pair differences between the two HSP70 genes, and thus the RNA probes

131 were likely to hybridize with both HSP70cB1i and HSP70cB2i mRNAs. The hybridization signals

132 increased in all tissues of heat-treated rotifers. Altogether, these results indicate that the B. plicatilis

133 HSP70cB1i and HSP70cB2i genes are heat-inducible and have similar expression profiles.

135 Phylogenetic Analysis of HSP70 Family Members

136 To assess the relationship of the B. plicatilis HSP70 genes with those of other rotifers and

137 other metazoans, we constructed gene trees of more than 100 HSP70 family members, including

138 members associated with the mitochondria and ER and many sequences known only from

139 automated genome annotation as "HSP70." To assess the robustness of the results we used

140 alignments made by three different methods [Clustal-Omega (Sievers et al. 2011), M-Coffee

141 (Wallace et al. 2006), and Expresso (Armougom et al. 2006)] to construct consensus trees with

142 maximum-likelihood (RAxML) and Bayesian (MrBayes) approaches. The RAxML

143 PROTGAMMAAUTO function found that the LG model of protein evolution (Le and Gascuel

144 2008) was optimal for all three alignments and this model was used to construct ML and Bayesian 
145 consensus trees. The six trees were in general agreement for major nodes; fig. 2 shows the Bayesian 146 consensus tree of the Expresso alignment and support for major nodes from each approach is shown 147 in table 1. In all analyses HSP70 family members known to be mitochondria- and ER-specific 148 formed two monophyletic groups (nodes 11 and 12 in fig. 2) in agreement with previous reports 149 (Boorstein et al. 1994; Miernyk 1997; Nikolaidis and Nei 2004). All family members known to be 150 cytosolic formed a third group (node 1; clades 1-6 in fig. 2), with yeast cytosolic HSP70s (clade 6)

151 basal to all metazoan cytosolic HSP70s. The tree topology generally supported the species 152 phylogeny. Within the metazoan cytosolic HSP70s (node 2; clades 1-5) there were two lineages:

153 Lineage A was well supported and contained of genes from arthropods, molluscs, and rotifers (node

1543 3; clade 1); Lineage B was more poorly resolved and represented by many diverse vertebrate and 155 invertebrate phyla (node 4; clades 2-5). There are two groups of vertebrate cytosolic HSP70s 156 (nodes 8 and 9), which form a monophyletic group (node 7; clade 5) in Bayesian and RAxML 157 analyses of Expresso and Clustal alignments. Unexpectedly, we found two additional clusters of 158 cytosolic HSP70 family members in all analyses: one composed of HSP70 genes from the phylum 159 Rotifera (node 5; clade 2) and the other from the phylum Nematoda (node 6; clade 3). This suggests 160 the evolution of two unique subgroups of HSP70, distinct from conventional vertebrate and 161 invertebrate HSP70s. We also constructed phylogenetic trees mainly using HSP70 family members 162 of known expression patterns (supplementary figs. S2 and S3). Both of the two HSP70 lineages as 163 well as most of the nodes described above were identified, supporting the robustness of the tree 164 shown in fig. 2, although several clades were poorly resolved in supplementary figs. 2 and 3 that 165 have limited number of samples.

166 The phylogenetic breadth of our analyses provided the opportunity to search for sequence 167 motifs characteristic of different groups within the HSP70 family. We identified a single region near 168 the $\mathrm{N}$ terminus (but after signal peptide motifs) that discriminates between metazoan mitochondrial, 169 ER, and cytosolic HSP70s (fig. 3), which enables better discrimination for organelle-specific 170 HSP70s together with the well-known C-terminal motifs for cytosolic (EEVD) and ER 
171 (HDEL/KDEL) HSP70s (supplementary fig. S4). We also found the RARFEEL motif, previously

172 described as possibly cytosolic-specific in conjunction with the EEVD motif (Cottin et al. 2008;

173 Simoncelli et al. 2010; Zheng et al. 2012), in several invertebrate genes that are found with high

174 confidence in the ER-associated node 12 (fig. 2; B. ibericus HSC70, C. gigas GRP, and B.

175 manjavacas HSC71). This result suggests that the RARFEEL is not a reliable predictor of cytosol

176 association. Indeed, this motif was identified by a comparison of few sequences (Lo et al. 2004) and

177 appears to have no strong scientific support.

178 Stress-inducible invertebrate HSP70s are reported to have an extra serine residue in their

179 ATPase domain (Kourtidis et al. 2006; Garbuz et al. 2011). In our analysis this insertion occurs only

180 in Lineage A irrespective of the stress-inducibility, where it is present in all sequences except $B$.

181 ibericus HSP70 and one of four Lottia HSP70s; in Priapulus HSP70-1 the serine has been replaced

182 by alanine (fig. 4). These results suggest that the extra serine residue is a characteristic of this

183 lineage and may be associated with the stress-inducibility of the ancestral HSP70, which will be

184 tested by broader sampling focusing on this residue. The alignment result supports the idea that

185 HSP70s from Nematoda and Rotifera evolved separately from the those in Annelida, Mollusca and

186 Arthropoda, which have been generally recognized as "invertebrate HSP70s."

188 Synonymous and Nonsynonymous Substitution Rates

189 Lastly, we calculated the synonymous and nonsynonymous substitution rates of 34

190 representative cytosolic HSP70 member genes. We primarily selected genes with known expression

191 patterns (stress-inducible or constitutive) from each clade, hypothesizing that constitutive HSP70

192 family members have been under stronger purifying selection compared to stress-inducible

193 members. Only well-conserved regions were used for the calculation (supplementary fig. S5). We

194 noted that B. ibericus HSP70 (GU574486) has an insertion at nucleotide position 403 and a

195 nucleotide deletion at position 563, causing a frame shift. Deduced amino acid sequences encoded

196 by nucleotides $403-563$ were markedly different from those of other HSP70 family members 
197 because of the frame shift, which resulted in the long genetic distance in phylogenetic trees (fig. 2).

198 Because the frame shift greatly affects the synonymous and nonsynonymous substitution rates, this 199 sequence was excluded from our analysis.

200 For the selected 34 sequences, we calculated synonymous and nonsynonymous substitution 201 rates (Ks and $\mathrm{Ka}$ ) for all combinations of representative cytosolic members of the HSP70 family

202 from metazoans (fig. 5A). Synonymous substitution rates (Ks) were saturated in many combinations

203 and only a limited number of combinations produced meaningful results (229 out of 465

204 combinations, supplementary material 1). The Ka/Ks values calculated for the 229 combinations

205 were averaged for each clade (fig. 5B). Overall, $\mathrm{Ka} / \mathrm{Ks}$ values were markedly lower than 1 ,

206 indicating that cytosolic HSP70 family member genes have been under purifying selection. The

207 purifying selection pressure appears to have been less in Lineage A (clade 1) than in Lineage B

208 (clades 2-5). The effect size between these lineages ranged from 0.19 to 1.64 (fig. 6C). Since the

209 effect size larger than 0.8 indicate large difference in multiple comparisons, these results support

210 our hypothesis that constitutive HSP70 family members [clade 4 and clade 5 (node 8)] have been

211 under stronger purifying selection.

212 Within Lineage B, the selection pressure was strongest in Nematode HSP70s (clade 3),

213 invertebrate HSC70/HSP70s (clade 4), and vertebrate HSC70s (clade 5, node 8). In particular,

214 vertebrate HSC70s (clade 5, node 8) and HSP70s (clade 5, node 9) showed significant difference in

215 the $\mathrm{Ka} / \mathrm{Ks}$ with a large effect size of 0.86 despite their close phylogenetic relationship. These results

216 also support the above hypothesis. To confirm how much this finding depends on the sampling, we

217 conducted the same analysis with a larger sample size, including most genes used in the

218 phylogenetic analysis, and obtained consistent results (supplementary fig. S6).

\section{Discussion}

221 A previous phylogenetic analysis by Kourtidis et al. (2006) classified metazoan cytosolic

222 HSP70s into three groups, invertebrate HSP70, vertebrate HSP70, and HSC70 including both 
223 vertebrate and invertebrate orthologues using data from a few taxa (Kourtidis et al. 2006). The

224 monophyletic origin of HSC70s was also proposed by Nikolaidis and Nei (2004) based on their

225 analysis using Drosophila and nematode sequences (Nikolaidis and Nei 2004). Surprisingly, to our

226 knowledge, there has been no significant update on the evolution of metazoan HSP70 family after

227 these small-scale studies despite the continuing expansion of available genomic data. Here we show

228 that there are at least two types of ancestral cytosolic HSP70 family member genes in all analyzed

229 phyla of metazoans, one (node 3; clade 1) giving birth to Lineage A of invertebrate HSP70s, and a

230 second (node 4; clade 5) to Lineage B of both vertebrate and invertebrate HSP70s and all HSC70

231 genes. This second lineage has further diversified within diverse phyla: Rotifera (node 5; clade 2),

232 Nematoda (node 6; clade 3), and Chordata (node 7; clade 5) (fig. 2), which has not been identified

233 in the previous reports. The tree topology is generally consistent with the species phylogeny,

234 indicating that the effect of sequence contamination is very limited.

235 Inter-clade comparison of the synonymous and nonsynonymous substitution rates shows that

236 while all HSP70 family members are under strong purifying selection, the pressure to conserve

237 amino acid sequence varies across clades. The presence of purifying selection has been reported for

238 mammalian (Hess et al. 2018), nematode (Nikolaidis and Nei 2004), and molluscan (Kourtidis et al.

239 2006) HSP70 genes, and our cross-phylum analysis was consistent with these reports. Most

240 dramatically, the canonical HSC70 genes of vertebrates (node 8; clade 5) showed stronger purifying

241 selection pressure than the HSP70 family members in node 9 (clade 5). This concept can be further

242 generalized by thoroughly investigating the saturation rate between each HSP70 gene (Pollock and

243 Larkin 2004). It is thus speculated that the role of HSC70 protein, the folding of newly synthesized

244 proteins, is more essential for cell survival compared to the role of the HSP70 cognates (node 9;

245 clade 5). In line with this speculation, cells deficient in the HSC70 gene are non-viable (Florin et al.

246 2004), whereas HSP70 knockout mice are mostly viable and fertile, although they are known to be

247 sensitive to stress (Daugaard et al. 2007). Meanwhile, genetic distance between each clade is not

248 uniform, and the gene conversion may also affect the accuracy of this calculation. In this regard our 
249 data should be interpreted in a semiquantitative manner.

250 It is known that nematode, mammalian, and molluscan HSP70 families have experienced gene 251 conversion events (Nikolaidis and Nei 2004; Kourtidis et al. 2006; Hess et al. 2018). In addition to

252 the purifying selection, gene conversion also likely contributes to the highly conserved nature of the

253 HSP70 family members found in this study. The tree topology might have been affected by the gene

254 conversion in closely related paralogues and orthologues such as human HSPA1A and HSPA1B

255 (Hess et al. 2018). However, gene conversion generally functions to conserve the sequence in a

256 species, and thus the overall topology of the metazoan tree is unlikely to be significantly affected.

257 Gene conversion rate should be about 10 times higher than the mutation rate to significantly affect

258 the topological distance according to a genomic simulation study (Touchon et al. 2009), but the

259 nucleotide identity between sequences is often lower than $70 \%$ in our phylogenetic analysis.

260 Given the presence of inducible and constitutively expressed genes in many clades of

261 metazoan cytosolic HSP70, it is difficult to predict the ancestral state of this phenotype, and

262 whether a gene is inducible or constitutive expression does not predict its relationship to other

263 family members. Phylogenetic analyses, the novel signature sequences near the $\mathrm{N}$ terminus (fig. 4),

264 and the extra serine residue (fig. 5) would provide better clues for the classification of HSP70

265 family members than their expression patterns under stress.

266 Based on the largest-ever cross-phylum phylogenetic analysis on HSP70 family, we propose a

267 new nomenclature that reflects the phylogenetic relationship of the HSP70 family members (fig. 6).

268 The proposed name of an HSP70 family member protein consists of "HSP70 + subcellular

269 localization + linage + copy number found in the organism + inducible or constitutive, if known."

270 For example, HSP70cA1i represents a cytosolic HSP70 in Lineage A, copy 1, inducible; and

271 HSP70mA1 for mitochondrial linage of unknown inducibility. Although the current study found

272 only one lineage in mitochondrial and ER members, the use of " $\mathrm{A}$ " in these organelle-specific

273 isoforms would be beneficial. Systematic lineage names such as HSP70cD and HSP70mB can be

274 assigned for novel lineages that may be discovered in future. Gene names are represented by 
275 lowercase italics of protein names (e.g., hsp70cb2i and hsp70eral).

276 In conclusion, the present study identified two novel HSP70 family members from a

277 monogonont rotifer, an emerging model in evolutionary biology. The subsequent phylogenetic

278 analyses illustrated the evolutionary history of the metazoan HSP70 family, in which stress

279 inducibility does not reflect evolutionary history. The proposed nomenclature based on molecular

280 evolution will help us understand the true nature of the HSP70 family.

282 Materials and Methods

283 Culture

284 We used the B. plicatilis Ishikawa strain (also called Brachionus sp. ISKW), originally

285 isolated from a Japanese eel culture pond (Yoshinaga et al. 2004), for cDNA cloning, semi-

286 quantitative RT-PCR, quantitative real-time PCR, and in situ hybridization. The cytochrome oxidase

287 subunit I gene sequence has been registered for detailed identification of this strain (GenBank

288 accession number LC422762). Rotifers were cultured at $25^{\circ} \mathrm{C}$ by a standard protocol (Kaneko et al.

289 2016) using half-diluted Brujewicz artificial seawater and fed the algae Chlorella regularis (Nikkai

290 Center, Tokyo, Japan) at a final concentration of approximately $7 \times 10^{6}$ cells $/ \mathrm{mL}$. See

291 supplementary material 2 for details of molecular characterization of rotifer HSP70 genes.

293 Phylogenetic Analysis

294 Metazoan HSP70 family genes were selected based on the following criteria: 1) genes from

295 diverse metazoan phyla are included; 2) HSP70 genes with known expression patterns are

296 prioritized; 3) both Lineage A and Lineage B genes from the same organism are included when

297 possible; and 4) the number of genes from a single species is limited to $\sim 5$ because within-phylum

298 gene duplications do not change the tree topology. Annotated and unannotated genome and

299 transcriptome databases were used. A fasta file containing sequences used for the tree is provided as

300 supplementary material 3. 
A multiple sequence alignment of HSP70 and HSC70 amino acid sequences was produced

302 using Clustal Omega, Expresso, and M-Coffee with default parameters. Bayesian phylogenetic

303 analysis was performed using MrBayes (v3.2.6) on the CIPRES scientific gateway v3.3 (Miller et

304 al. 2010) with four chains each for $10^{7}$ generations. Every 1000th trees were sampled, and the first

$30525 \%$ of samples were discarded as a burn-in. Bayesian consensus trees were visualized with the

306 TreeView software v1.4.3.

308 Synonymous and Nonsynonymous Substitution Rates

$309 \quad \mathrm{R}$ version 4.0.4 was used for the calculation of the synonymous and nonsynonymous

310 substitution rates (the kaks function), Kruskal-Wallis test (kruskal.test function), and post-hoc test

311 (pairwise.wilcox.test function) on the Macintosh platform. Heat maps were created in Microsoft

312 Excel 2016 (Redmond, MA).

\section{Author Contributions}

315 Conceived and designed the experiments: TY, GK. Performed experiments and data analyses: EMY,

316 FLJ, DMW, GK. Contributed reagents/materials/analysis tools: EH, DMW, GK. Wrote the paper:

317 DMW, GK. Read and approved the final manuscript: EMY, TY, FLJ, EH, DMW, GK.

319 Acknowledgements

320 We express our sincere thanks to Dr. Kristin Gribble, Marine Biological Laboratory, for helpful 321 discussions and comments on the manuscript. The authors are grateful to Cynthia Rodriguez and 322 Jamie Wilson, University of Houston-Victoria, for their help in gene expression analyses. GK was 323 supported by funding from M.G. and Lillie A. Johnson Foundation, Victoria, Texas.

\section{Figure Legends}

326 Fig. 1. Molecular characterization of Brachionus plicatilis HSP70 genes. (A-C) Semi-quantitative 
327 reverse transcription-PCR. A representative result is shown out of three independent experiments.

328 Location of primers rHSP70_gapF and rHSP70_gapR that amplify cDNA fragment of 112 and 124

329 bp from HSP70cB1i and HSP70cB2i cDNAs, respectively (A). Polyacrylamide gel electrophoresis

330 patterns of the RT-PCR product. Cycle numbers within a linear range of PCR amplification were

331 determined to be 24 to 28 cycles for both cDNAs by preliminary experiments on the basis of signal

332 intensities of amplified products by RT-PCR (B). Signal intensities of HSP70cB1i and HSP70cB2i

333 genes standardized to those of $\beta$-actin (C). (D) Heat stress-induced expression of HSP70 quantified

334 by real-time PCR. Beta-actin gene was used as the internal control. Bars represent standard errors

335 from three replications. One-way ANOVA detected significant effects of heat treatment $(\mathrm{F}=3.281$,

$336 \mathrm{df}=5, P=0.0426)$. Dunnett's multiple comparison was used to detect significant differences

337 between the control and other groups. Difference between control and two hours group was

338 statistically significant $(\mathrm{t}=3.246, P=0.028)$. (E) In situ hybridization for $B$. plicatilis HSP70

339 genes. Rotifers were fixed $4 \mathrm{~h}$ after the heat treatment.

341 Fig. 2. Bayesian consensus tree of HSP70 family members. Nodes discussed in the text are

342 indicated. Numbers on the branches indicate the posterior probability support for each node. Stress-

343 inducible and constitutive genes are shown in red and blue, respectively. No data on the stress

344 inducibility is available for genes shown in black. Approved HGNC names of human HSP70 family

345 members are shown in yellow boxes for reference. The DDBJ/EMBL/GenBank accession numbers

346 and other information are summarized in supplementary table S2. Clades 1 to 6 include only

347 cytosolic members of the HSP70 family.

349 Fig. 3. Logo visualization of mitochondrial-, endoplasmic reticulum-, and cytosolic-specific

350 sequence near the N terminus of the HSP70 family members. Compared to the cytosolic form, ER-

351 specific forms have several conserved amino acid substitutions: Q8K, H9N, K11R, and T23I.

352 Similarly, mitochondrial forms have Y1N, G5A, F7M, Q8E, H9G, G10K, K11T/Q, V12P/A, E13K, 
I14V, I15L, A16E, D17A, and Q18E.

Fig. 5. Comparison of the amino acid sequences of the HSP70 family members from invertebrates.

356 Nodes in the phylogenetic tree (fig. 3) are indicated on the right margin, and serine residues specific

357 to node 3 are highlighted. Stress-inducible and non-inducible genes are shown in red and blue,

358 respectively. No data on the stress inducibility is available for genes shown in black. The accession

359 numbers and other information are summarized in supplementary table S2.

361 Fig. 6. Selection pressure on cytosolic HSP70 family member genes. (A) Nonsynonymous

362 substitution rates (Ka values) between 34 HSP70 family member genes. Gene number 1 is the fruit

363 fly HSP70 Bb (AF295957) gene. The substitution rate of fruit fly HSP70 Aa and Bb gene was

364 00395526, which is shown as 0.00 in the figure. Accession numbers for other genes are indicated in

365 the figure. Clades and nodes correspond to those in fig. 2. (B) Bee swarm boxplots of Ka/Ks values

366 for each clade. Only Ka/Ks values calculated from inter-cluster pairs were averaged. Statistical

367 differences were calculated by Kruskal-Wallis test (chi-squared $=88.704, \mathrm{df}=5, \mathrm{p}<2.2 \mathrm{e}-16$ )

368 followed by the non-parametric post-hoc tests (pairwise Wilcox test with P value adjustment by the

369 Holm method). Clades sharing same letters are not significantly different at the $5 \%$ level of

370 significance. One outlier in clade $3(0.53)$ is not included in the plot (see supplementary material 1 )

371 although this value was used for all statistical analyses. (C) Effect sizes (Cohen's d) for all

372 comparisons. Combinations where significant differences were found in the pairwise Wilcox test are

373 shown in bold.

\section{$375 \quad$ References}

376 Armougom F, Moretti S, Poirot O, Audic S, Dumas P, Schaeli B, Keduas V, Notredame C. 2006.

377 Expresso: automatic incorporation of structural information in multiple sequence alignments using 378 3D-Coffee. Nucleic Acids Res 34:W604-W608.

379 Baird NA, Turnbull DW, Johnson EA. 2006. Induction of the heat shock pathway during hypoxia 
380 requires regulation of heat shock factor by hypoxia-inducible factor-1. J Biol Chem 281:38675-

38138681.

382 Boorstein WR, Ziegelhoffer T, Craig EA. 1994. Molecular evolution of the HSP70 multigene 383 family. J Mol Evol 38:1-17.

384 Ceyhun SB, Sentürk M, Ekinci D, Erdoğan O, Ciltaş A, Kocaman EM. 2010. Deltamethrin

385 attenuates antioxidant defense system and induces the expression of heat shock protein 70 in

386 rainbow trout. Comp Biochem Physiol, C Toxicol Pharmacol 152:215-223.

387 Cottin D, Ravaux J, Leger N, Halary S, Toullec JY, Sarradin PM, Gaill F, Shillito B. 2008. Thermal

388 biology of the deep-sea vent annelied Paralvinella grasslei: in vivo studies. J Exp Biol 211:2196-

3892204.

390 Daugaard M, Rohde M, Jäättelä M. 2007. The heat shock protein 70 family: Highly homologous

391 proteins with overlapping and distinct functions. FEBS Lett 581:3702-3710.

392 De Nadal E, Ammerer G, Posas F. 2011. Controlling gene expression in response to stress. Nat Rev

393 Genet 12:833-845.

394 Demand J, Lüders J, Höhfeld J. 1998. The carboxy-terminal domain of Hsc70 provides binding

395 sites for a distinct set of chaperone cofactors. Mol Cell Biol 18:2023-2028.

396 Florin L, Becker KA, Sapp C, Lambert C, Sirma H, Müller M, Streeck RE, Sapp M. 2004. Nuclear

397 translocation of papillomavirus minor capsid protein L2 requires Hsc70. J Virol 78:5546-5553.

398 Fröbius AC, Funch P. 2017. Rotiferan Hox genes give new insights into the evolution of metazoan

399 bodyplans. Nat Commun 8:9.

400 Garbuz D. 2017. Regulation of heat shock gene expression in response to stress. Mol Biol 51:352-

401367.

402 Garbuz DG, Yushenova IA, Zatsepina OG, Przhiboro AA, Bettencourt BR, Evgen\&apos;ev MB.

403 2011. Organization and evolution of hsp70 clusters strikingly differ in two species of Stratiomyidae

404 (Diptera) inhabiting thermally contrasting environments. BMC Evol Biol 11:74.

405 Hartl FU, Bracher A, Hayer-Hartl M. 2011. Molecular chaperones in protein folding and

406 proteostasis. Nature 475:324-332.

407 Hess K, Oliverio R, Nguyen P, Le D, Ellis J, Kdeiss B, Ord S, Chalkia D, Nikolaidis N. 2018.

408 Concurrent action of purifying selection and gene conversion results in extreme conservation of the 409 major stress-inducible Hsp70 genes in mammals. Sci Rep 8:1-16. 
410 Jayasena S, Chandrasekharan N, Karunanayake EH. 1999. Molecular characterisation of a hsp70

411 gene from the filarial parasite Setaria digitata. Int J Parasitol 29:581-591.

412 Jedlicka P, Mortin MA, Wu C. 1997. Multiple functions of Drosophila heat shock transcription

413 factor in vivo. EMBO J 16:2452-2462.

414 Judge ML, Botton ML, Hamilton MG. 2011. Physiological consequences of the supralittoral fringe:

415 microhabitat temperature profiles and stress protein levels in the tropical periwinkle Cenchritis

416 muricatus (Linneaus, 1758). Hydrobiologia 675:143-156.

417 Kaneko G, Yoshinaga T, Gribble KE, Mark Welch D, Ushio H. 2016. Measurement of survival time

418 in Brachionus rotifers: synchronization of maternal conditions. J Vis Exp 113:e54126.

419 Kourtidis A, Drosopoulou E, Nikolaidis N, Hatzi VI, Chintiroglou CC, Scouras ZG. 2006.

420 Identification of several cytoplasmic HSP70 genes from the Mediterranean mussel (Mytilus

421 galloprovincialis) and their long-term evolution in Mollusca and Metazoa. J Mol Evol 62:446-459.

422 Le SQ, Gascuel O. 2008. An improved general amino acid replacement matrix. Mol Biol Evol

$423 \quad 25: 1307-1320$.

424 Liu L, Cheng T-y, Yang Y. 2017. Cloning and expression pattern of a heat shock cognate protein 70

425 gene in ticks (Haemaphysalis flava). Parasitol Res 116:1695-1703.

426 Lo W-Y, Liu K-F, Liao I-C, Song Y-L. 2004. Cloning and molecular characterization of heat shock

427 cognate 70 from tiger shrimp (Penaeus monodon). Cell Stress Chaperones 9:332.

428 Luan W, Li F, Zhang J, Wen R, Li Y, Xiang J. 2010. Identification of a novel inducible cytosolic

429 Hsp70 gene in Chinese shrimp Fenneropenaeus chinensis and comparison of its expression with the

430 cognate Hsc70 under different stresses. Cell Stress Chaperones 15:83-93.

431 Marchler G, Wu C. 2001. Modulation of Drosophila heat shock transcription factor activity by the 432 molecular chaperone DROJ1. EMBO J 20:499-509.

433 Miernyk JA. 1997. The $70 \mathrm{kDa}$ stress-related proteins as molecular chaperones. Trends Plant Sci

$434 \quad 2: 180-187$.

435 Miller MA, Pfeiffer W, Schwartz T. 2010. Creating the CIPRES Science Gateway for inference of

436 large phylogenetic trees. Proceedings of the Gateway Computing Environments Workshop (GCE);

437 New Orleans, LA. p. 1-8.

438 Nikolaidis N, Nei M. 2004. Concerted and nonconcerted evolution of the Hsp70 gene superfamily

439 in two sibling species of nematodes. Mol Biol Evol 21:498-505. 
440 Piano A, Asirelli C, Caselli F, Fabbri E. 2002. Hsp70 expression in thermally stressed Ostrea edulis,

441 a commercially important oyster in Europe. Cell Stress Chaperones 7:250.

442 Pollock DD, Larkin JC. 2004. Estimating the degree of saturation in mutant screens. Genetics

$443 \quad 168: 489-502$.

444 Sanders BM. 1993. Stress proteins in aquatic organisms - an environmental perspective. Crit Rev

445 Toxicol 23:49-75.

446 Sievers F, Wilm A, Dineen D, Gibson TJ, Karplus K, Li W, Lopez R, McWilliam H, Remmert M,

447 Söding J. 2011. Fast, scalable generation of high-quality protein multiple sequence alignments using

448 Clustal Omega. Mol Syst Biol 7:539.

449 Simoncelli F, Morosi L, Di Rosa I, Pascolini R, Fagotti A. 2010. Molecular characterization and

450 expression of a heat-shock cognate 70 (Hsc70) and a heat-shock protein 70 (Hsp70) cDNAs in Rana

451 (Pelophylax) lessonae embryos. Comp Biochem Physiol, A Mol Integr Physiol 156:552-560.

452 Sørensen JG, Kristensen TN, Loeschcke V. 2003. The evolutionary and ecological role of heat

453 shock proteins. Ecol Lett 6:1025-1037.

454 Sorger PK, Pelham HR. 1988. Yeast heat shock factor is an essential DNA-binding protein that

455 exhibits temperature-dependent phosphorylation. Cell 54:855-864.

456 Struck TH, Wey-Fabrizius AR, Golombek A, Hering L, Weigert A, Bleidorn C, Klebow S,

457 Iakovenko N, Hausdorf B, Petersen M. 2014. Platyzoan paraphyly based on phylogenomic data

458 supports a noncoelomate ancestry of Spiralia. Mol Biol Evol 31:1833-1849.

459 Touchon M, Hoede C, Tenaillon O, Barbe V, Baeriswyl S, Bidet P, Bingen E, Bonacorsi S, Bouchier

460 C, Bouvet O. 2009. Organised genome dynamics in the Escherichia coli species results in highly

461 diverse adaptive paths. PLoS genet 5:e1000344.

462 Wallace IM, O'sullivan O, Higgins DG, Notredame C. 2006. M-Coffee: combining multiple

463 sequence alignment methods with T-Coffee. Nucleic Acids Res 34:1692-1699.

464 Yabu T, Imamura S, Mohammed MS, Touhata K, Minami T, Terayama M, Yamashita M. 2011.

465 Differential gene expression of HSC70/HSP70 in yellowtail cells in response to chaperone-

466 mediated autophagy. FEBS J 278:673-685.

467 Yoshinaga T, Minegishi Y, Rumengan IFM, Kaneko G, Furukawa S, Yanagawa Y, Tsukamoto K,

468 Watabe S. 2004. Molecular phylogeny of the rotifers with two Indonesian Brachionus lineages.

469 Coast Mar Sci 29:45-56. 
bioRxiv preprint doi: https://doi.org/10.1101/2020.09.21.307264; this version posted April 22, 2021. The copyright holder for this preprint (which

was not certified by peer review) is the author/funder, who has granted bioRxiv a license to display the preprint in perpetuity. It is made available under aCC-BY-NC-ND 4.0 International license.

470 Zheng G, Dong S, Hou Y, Yang K, Yu X. 2012. Molecular characteristics of HSC70 gene and its

471 expression in the golden apple snails, Pomacea canaliculata (Mollusca: Gastropoda). Aquaculture

472 358-359:41-49.

473 Zuiderweg ER, Hightower LE, Gestwicki JE. 2017. The remarkable multivalency of the Hsp70

474 chaperones. Cell Stress Chaperones 22:173-189.

475 
Table 1. Support for major nodes from each approach

\begin{tabular}{|l|l|l|l|l|l|l|l|}
\hline & & \multicolumn{2}{|l|}{ ClustalOmega } & Expresso & \multicolumn{2}{l|}{ M-Coffee } \\
\hline & & MrBayes & RAxML & MrBayes & RAxML & MrBayes & RAxML \\
\hline 1 & Cytosolic & 100 & 99 & 100 & 99 & 100 & 100 \\
\hline 2 & Metazoan & 100 & 95 & 100 & 99 & 100 & 94 \\
\hline 3 & Lineage 1 & $(\mathrm{a})$ & 99 & 100 & 99 & 100 & 100 \\
\hline 4 & Lineage 2 & $(\mathrm{b})$ & 41 & 85 & 67 & $(\mathrm{~b})$ & $(\mathrm{c})$ \\
\hline 5 & Rotifer & 100 & 48 & 74 & 48 & 100 & 65 \\
\hline 6 & Nematode & 94 & $93(\mathrm{~d}, \mathrm{e})$ & $100(\mathrm{e})$ & $96(\mathrm{e})$ & $100(\mathrm{e})$ & $86(\mathrm{e})$ \\
\hline 7 & Chordate & & 42 & 85 & 35 & & \\
\hline 8 & HSC & 100 & 91 & 100 & 86 & 100 & 31 \\
\hline 9 & HSP & 100 & 88 & 100 & 83 & 100 & 87 \\
\hline 10 & Outgroup & 100 & 99 & 100 & 99 & 100 & 100 \\
\hline 11 & Mitochondrial & 100 & 100 & 100 & 97 & 100 & 100 \\
\hline 12 & ER & 100 & 99 & 100 & 100 & 100 & 100 \\
\hline
\end{tabular}

(a) Rotifer genes (node 5) is included in node 3 with $83 \%$ support

(b) Rotifer genes (node 5) sister group to node 3 with $80 \%$ support, other paraphyly

(c) Nematode genes (node 6) basal; rotifer genes sister group to node 3 with $46 \%$ support, other paraphyly

(d) $36 \%$ with Trichuris (immediate outgroup to node 6 )

(e) Trichuris not in node 6 


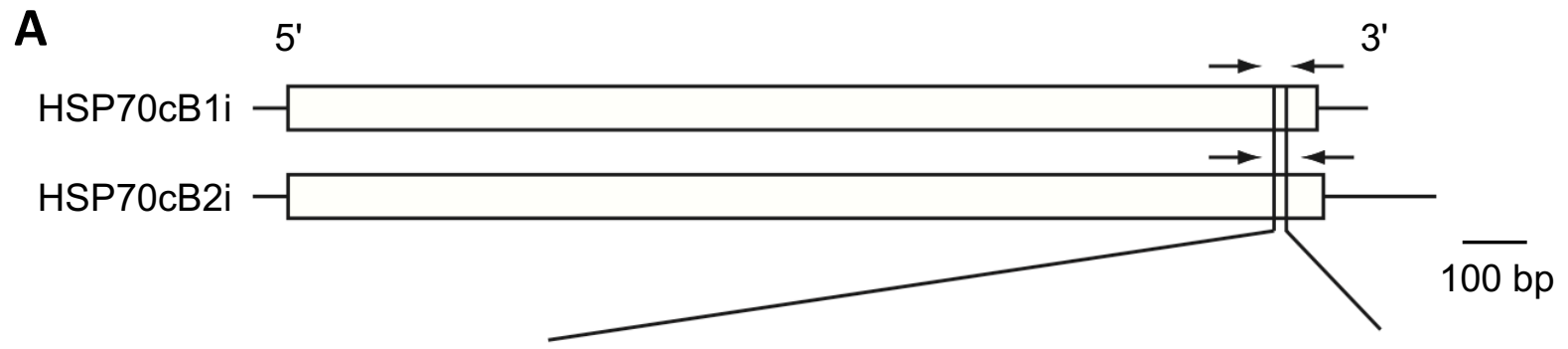

GTGGCATGCCTGGTGGTATGCCTGG-----------CGGTATGCCTGGTGGAATGCCTG 1968

GTGGTATGCCTGGTGGTATGCCTGGTGGTATGCCGGGCGGTATGCCTGGTGGAATGCCTG 1979

B
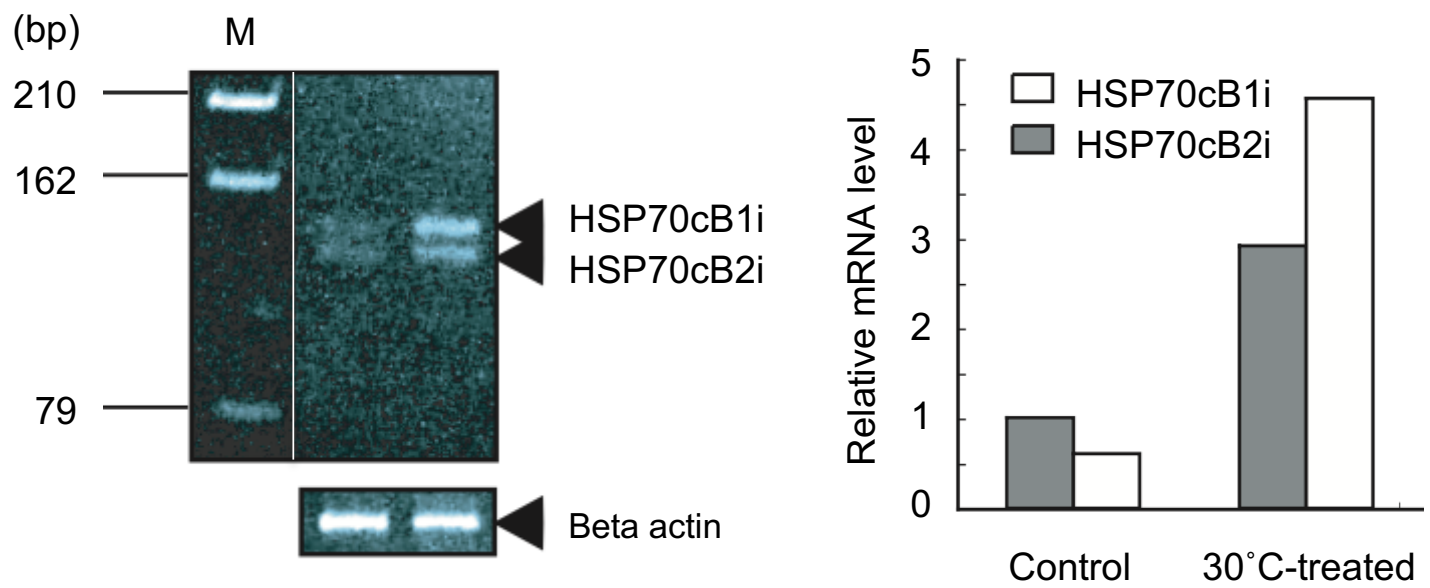

D

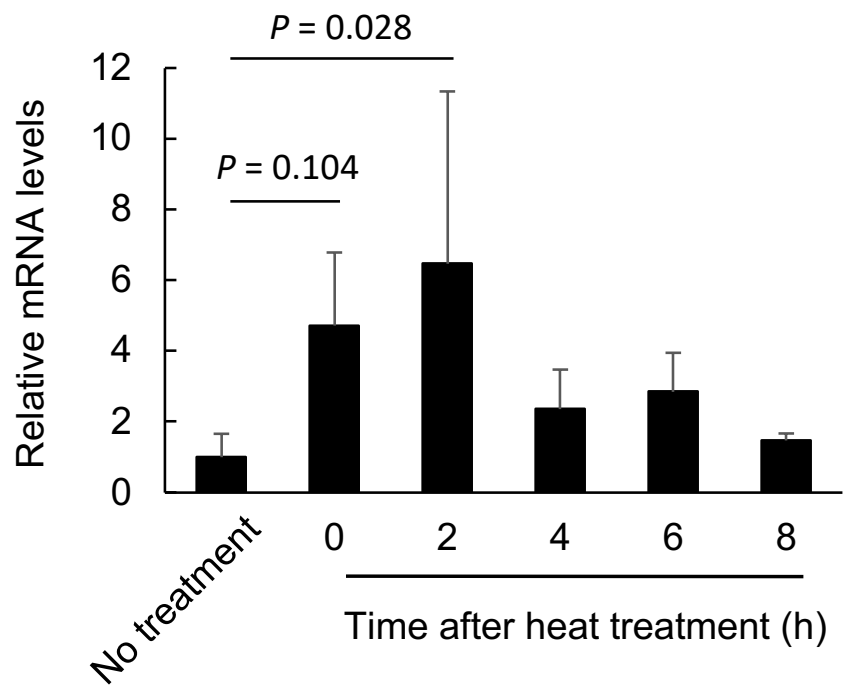

E

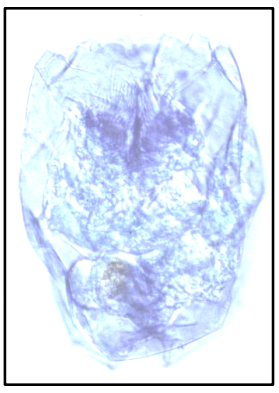

Control

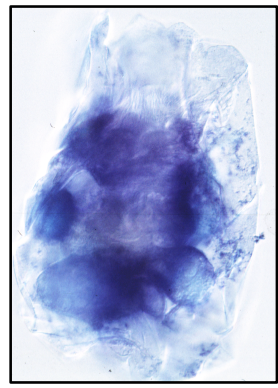

$30^{\circ} \mathrm{C}$-treated 
Cytosol

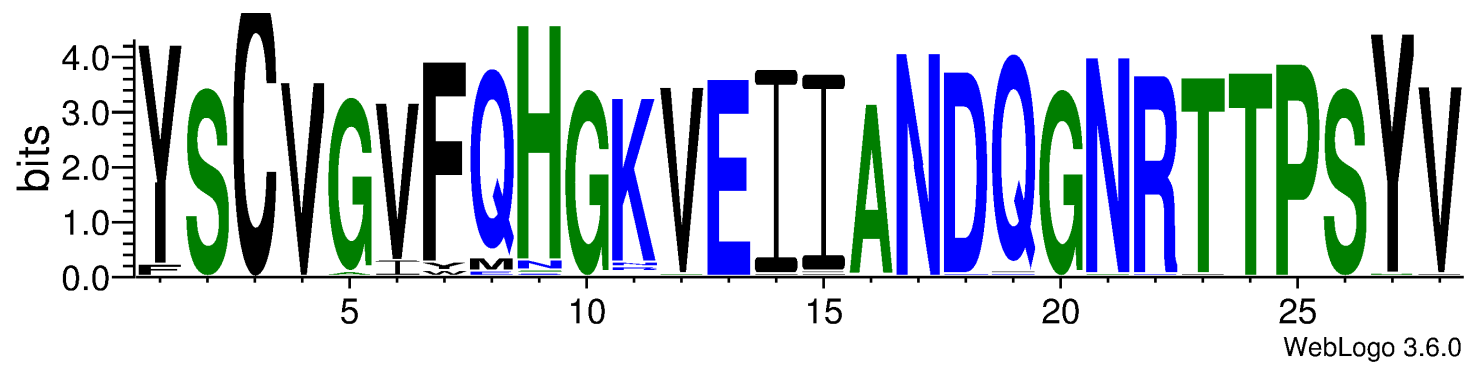

Endoplasmic reticulum

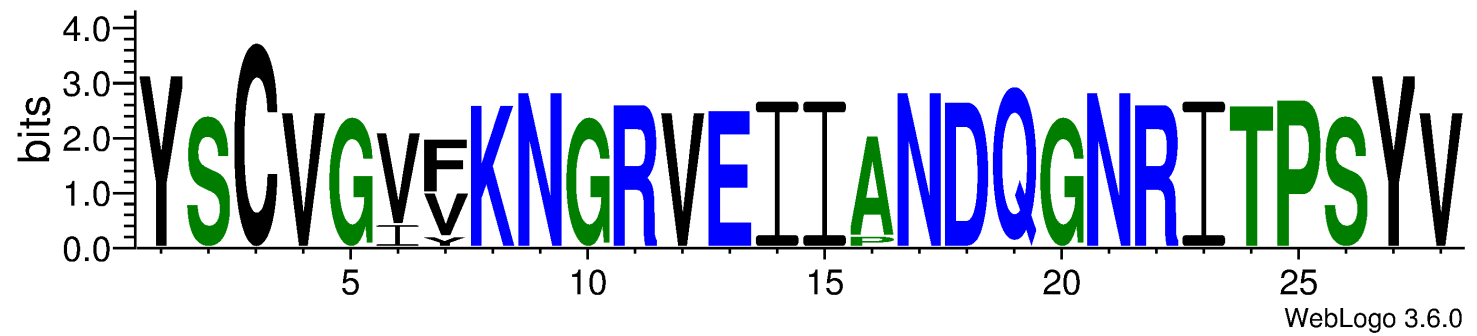

Mitochondrial

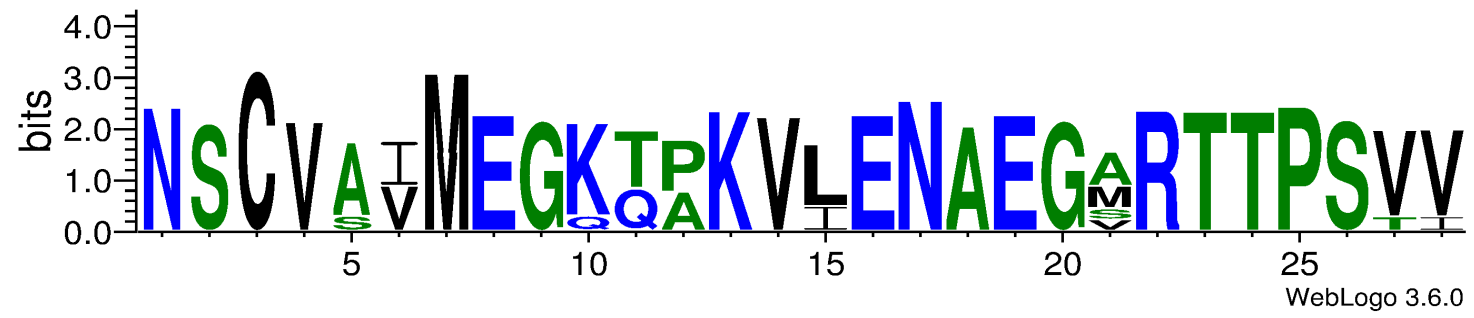


D. melanogaster HSP70A D. melanogaster HSP70B Bombyx HSP70

Spodoptera HSP70

Paralvinella HSP70-1

Lingula HSP71

A. vaga HSP70-1B

A. vaga HSP70-1A

$B$. ibericus HSP70

Priapulus HSP70-1

Aplysia HSP70-1

Aplysia HSP70-2

Crassostrea HSP70

Lottia HSP70-4

Lottia HSP70-1

Lottia HSP70-3

Lottia HSP70-2

Haliotis HSP70

B. plicatilis HSP70-1

B. manjavacas HSP70-1

A. vaga HSP70-2A

C. elegans HSP70A

Wuchereria HSP70

Setaria HSP70

Strongyloides HSP70

Schistosoma HSP70-2

M. nipponense HSP70-1

Mytilus HSP70

Tevnial HSP70-1

Perinereisl HSP70-1

Lottia HSP70c

Dugesia HSP70

Oncorhynchus HSC71

H. sapiens HSC71

Pelodiscus HSC70

H. sapiens HSP70-1B

H. sapiens HSP70-like1

$X$. maculatus HSP70-1

Alligator HSP70A
KGERNVLIFDLGGGTFDVSILTIDE----GSLFEVRSTAGDT 224 KGERNVLILDLGGGTFDVSILTIDE----GSLFEVRSTAGDT 224 KGERNVLIFDLGGGTFDVSILTIDE----GSLFEVKSTAGDT 224 KGERNVLIFDLGGGTFDVSILTIDE----GSLFEVRATAGDT 224 SGEKNVLIFDLGGGTFDVSILSIAD----GSLFEVKSTAGDT 229 QGEKNVLIFDLGGGTFDVSILSIAD----GSLFEVRSTAGDT 228 HRDQNILIYDLGGGTFDVSVLTISGGDVSGSVFEVKATAGDT 238 QRDQNILIYDLGGGTFDVSILTISGGDAGGSVFEVRSTAGDT 238 KRRKNVLIYDLGGGTFDVSVLSIND----G-LFEVLSTNGNT 224 AGERNVLVYDLGGGTFDVSVLTIDE----GAMFEVRSTAGDT 226 KGEKHVLIFDLGGGTFDVSVLAIDE----GSIFEVKATAGDT 227 SGEKNVLIFDLGGGTFDVSVLTIDE----GSMFEVKATAGDT 230 SGEKNVLIFDLGGGTFDVSILTIDE----GSIFEVRSTAGDT 228 DTEQHILIFDLGGGTFDVSILAIED----G-VFEVLATAGDT 227 SGEKNVLIFDLGGGTFDVSILTIDE----GSLFEVKSTAGDT 225 SGEKNVLIFDLGGGTFDVSILTIDE----GSLFEVKSTAGDT 226 SGEKNVLIFDLGGGTFDVSILAIDE----GSLFEVKSTAGDT 226 KGEKNVLIFDLGGGTFDVPVLTIDE----GSMFEVRSTAGDT 231 GGEKNILIFDLGGGTFDVSILTIEE----G-IFEVKSTAGDT 226 AGEKNILIFDLGGGTFDVSILTIED----G-IFEVKATAGDT 226 SGERNILIFDLGGGTFDVSVLKIEE----G-IFEVKSTAGDT 184 HGERNVLIFDLGGGTFDVSILTIED----G-IFEVKSTAGDT 227 HGERNVLIFDLGGGTFDVSILTIED----G-IFEVKSTAGDT 226 HGERNVLIFDLGGGTFDVSILTIED----G-IFEVKSTAGDT 226 ATERNVLIFDLGGGTFDVSVLTIED----G-IFEVKSTAGDT 227 GGERNVLIFDLGGGTFDVSILTIED----G-IFEVKSTAGDT 224 GGERNVLIFDLGGGTFDVSILTIED----D-IFEVKSTAGDT 226 GGERNVLIFDLGGGTFDVSILTIKD----G-IFEVKSTSGDT 227 GGEQNVLIFDLGGGTFDVSILTIED----G-IFEVKSTAGDT 226 GGERNVLIFDLGGGTFDVSILTIED----G-IFEVKSTAGDT 225 GGERNVLIFDLGGGTFDVSILTIED----G-IFEVKSTAGDT 226 GIERNVLIFDLGGGTFDVSILTIED----G-IFEVKSTAGDT 226 GAERNVLIFDLGGGTFDVSILTIED----G-IFEVKSTAGDT 226 GAERNVLIFDLGGGTFDVSILTIED----G-IFEVKSTAGDT 226 GAERNVLIFDLGGGTFDVSILTIED----G-IFEVKSTAGDT 226 KGERNVLIFDLGGGTFDVSILTIDD----G-IFEVKATAGDT 226 QGERHVLIFDLGGGTFDVSILTIDD----G-IFEVKATAGDT 228 RGERNVLIFDLGGGTFDVSILTIED----G-IEEVKSTAGDT 228 AGEKNVLIFDLGGGTLDVSILTIED----G-IFEVKSTAGDT 229 


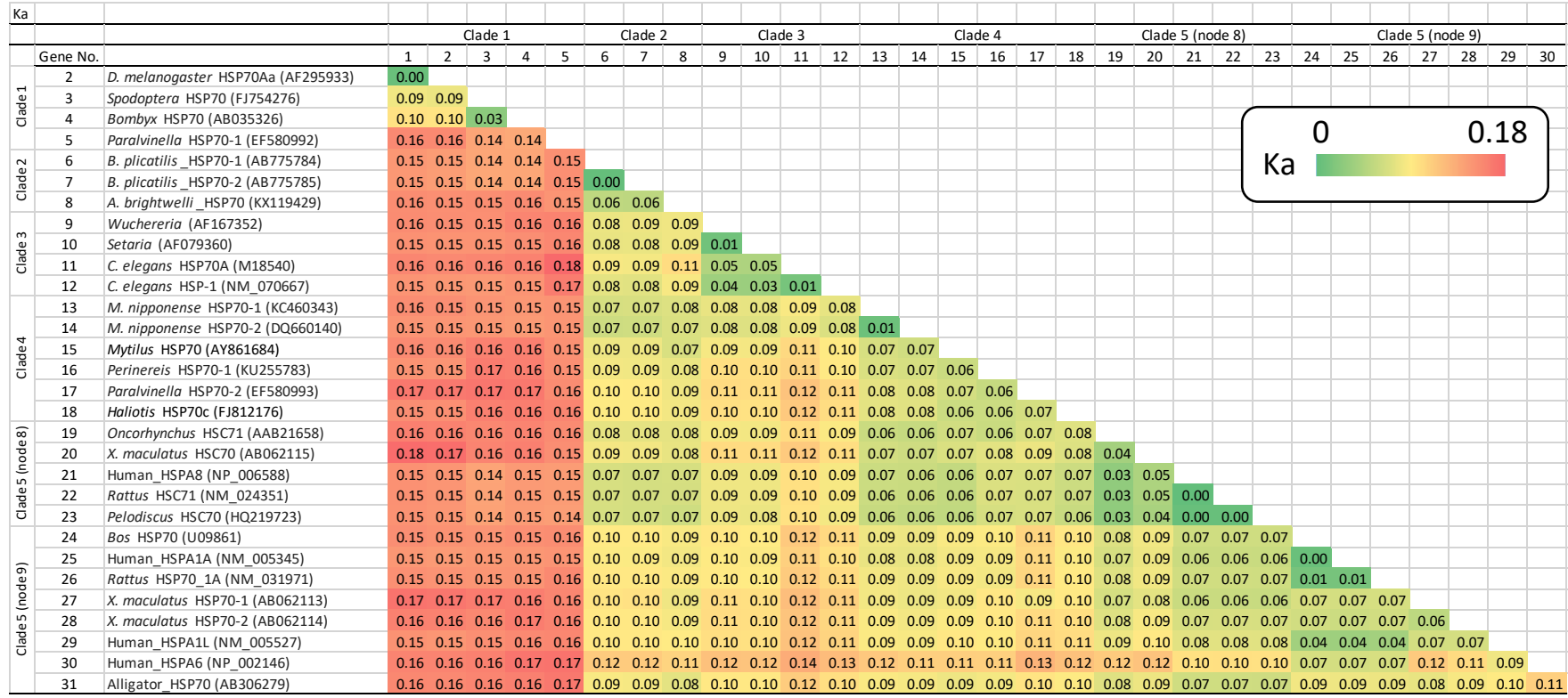

B

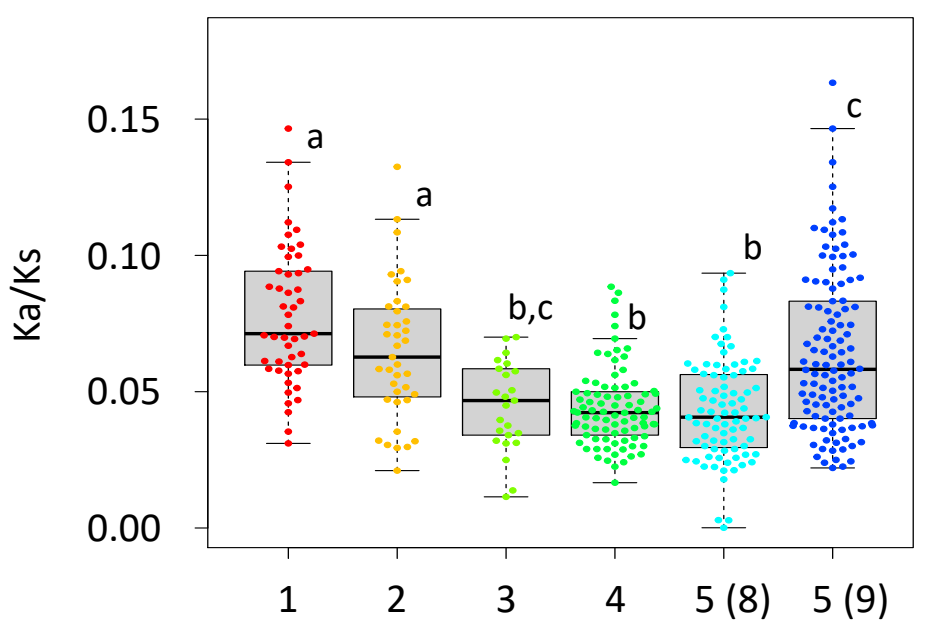

Clade (node)
C

Clade (node)

\begin{tabular}{|c|c|c|c|c|c|}
\hline & 2 & 3 & 4 & $5(8)$ & $5(9)$ \\
\hline 1 & 0.51 & 0.19 & 1.64 & 1.55 & 0.49 \\
\hline 2 & & 0.01 & 1.01 & 0.98 & 0.02 \\
\hline 3 & & & 0.28 & 0.29 & 0.01 \\
\hline 4 & & & & 0.05 & 0.87 \\
\hline $5(8)$ & & & & & 0.86 \\
\hline
\end{tabular}

\section{1: Invertebrate HSP70s (Lineage A)}

2: Rotifera HSP70s (Lineage B)

3: Nematode HSP70s (Lineage B)

4: Invertebrate HSP/HSC70s (Lineage B)

5 (8): Vertebrate HSC70s (Lineage B)

5 (9): Vertebrate HSP70s (Lineage B) 


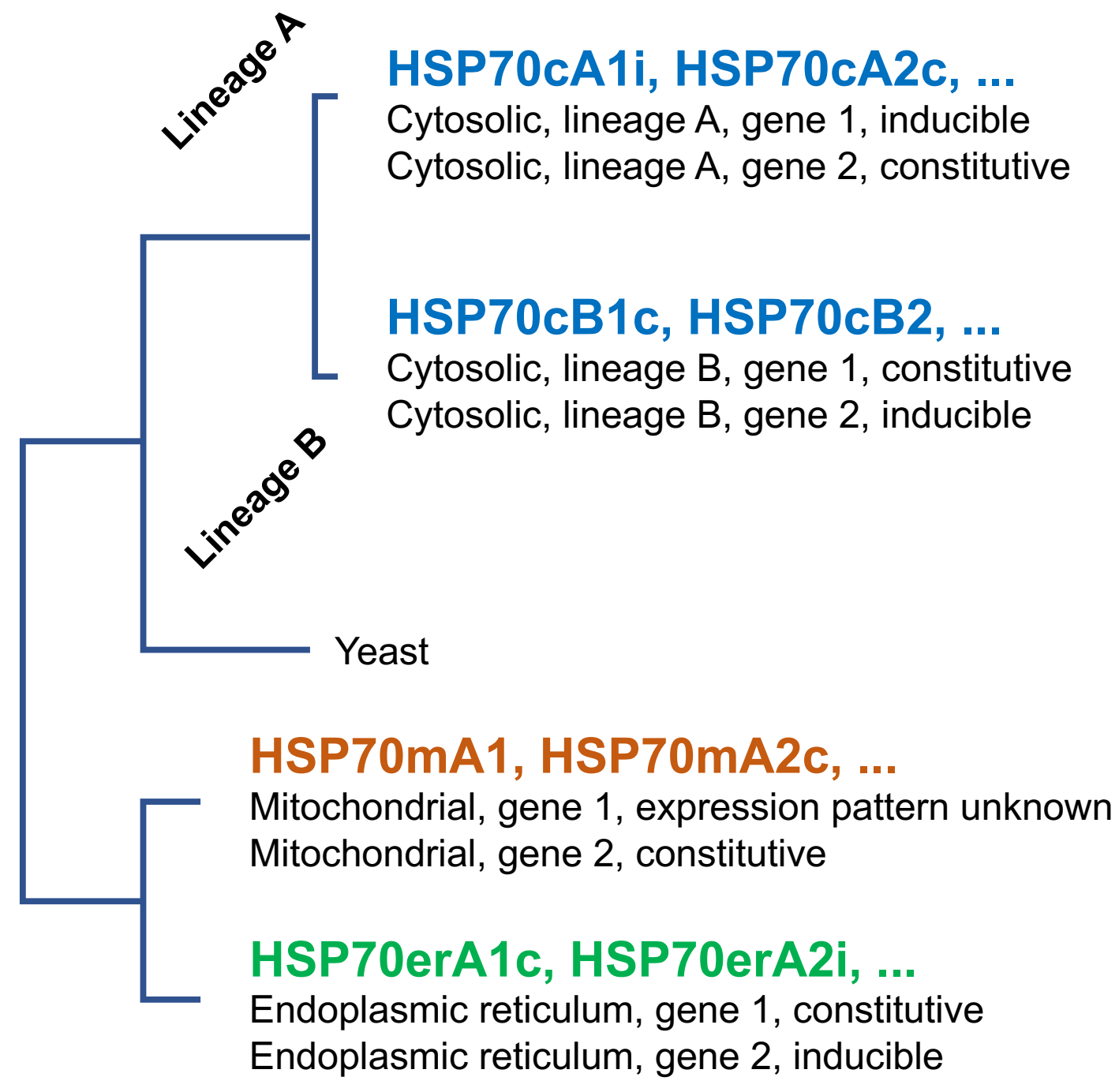


Table 1. Nucleotide sequences of primers used in this study

\begin{tabular}{|c|c|c|c|}
\hline Primer name & Sequence $\left(5^{\prime}-3^{\prime}\right)$ & Nucleotide position & Gene \\
\hline \multicolumn{4}{|l|}{ cDNA cloning } \\
\hline rHSP70_5RACE1 & AAAAGTCCTGGAGCAGCTT & $1107-1125^{*}$ & HSP70-1, 2 \\
\hline rHSP70_5RACE2 & GAACTCCTCGACAAAATGGTT & $780-800^{*}$ & HSP70-1, 2 \\
\hline rHSP70_5RACE3 & CAGGTCAAAGATCAGGATGTT & $645-665^{*}$ & HSP70-1, 2 \\
\hline rHSP70_3RACE1 & AGGCCAACATCGAAATAGA & $901-919 *$ & HSP70-1, 2 \\
\hline rHSP70_3RACE2 & GACAAGTCAAGTGTGAACGAG & $1044-1064 *$ & HSP70-1, 2 \\
\hline rHSP70_full_f1 & ACATACACCCAGACACACAA & $1-20 *$ & HSP70-1, 2 \\
\hline rHSP70_full_r1 & GTCGACTTCATCAATGGTGG & $2002-2021^{*}$ & HSP70-1, 2 \\
\hline GeneRacer 5' RACE & CGACTGGAGCACGAGGACACTGA & - & - \\
\hline GeneRacer 5' RACE Nested & GGACACTGACATGGACTGAAGGAGTA & - & - \\
\hline Oligo-dT adapter & GGCCACGCGTCGACTAGTAC $(\mathrm{T})_{17}$ & - & - \\
\hline AUAP & GGCCACGCGTCGACTAGTAC & - & - \\
\hline \multicolumn{4}{|l|}{ Semi-quantitative $R T-P C R$} \\
\hline rHSP70_gapF & CGATTATTACTCAGTTGTATCAG & $1879-1901 *$ & HSP70-1, 2 \\
\hline rHSP70_gapR & ATCAATGGTAGGTCCACTGC & $1993-2012 *$ & HSP70-1, 2 \\
\hline rActinF & CCTGCCATGTACGTAGCC & 2-19 & Actin \\
\hline rActinR & ATCACTTGACCATCGGGC & $340-357$ & Actin \\
\hline \multicolumn{4}{|l|}{ Quantitative real-time $P C R$} \\
\hline RT-hsp70-140F & AGTGGAAATAATCGCCAACGA & $140-160 *$ & HSP70 \\
\hline RT-hsp70-208R & TCGGTGTCGGTGAACGCTA & $190-208 *$ & HSP70 \\
\hline RT-AC-306F & CGTCCAGTTCCTCGTTGGAA & $306-325$ & Actin \\
\hline RT-AC-373R & CTCGTTGCCAATGGTGATCA & $354-373$ & Actin \\
\hline \multicolumn{4}{|l|}{ In situ hybridization } \\
\hline rHSP70_insituF & CCATTTACAAACACAAATATT & 2026-2046* & HSP70-1, 2 \\
\hline rHSP70_insituR & GTCAAACAAATCTTTTATC & 2085-2103* & HSP70-1, 2 \\
\hline
\end{tabular}

*Nuceltide positions in HSP70-1 gene 
$\checkmark$ family members used for the construction of phylogenetic trees

\begin{tabular}{|c|c|c|c|c|c|c|c|c|}
\hline Organism and protein name & Proposed name & Nucleotide accession number & Protein ID & Tissue & Treatment & Quantification method & Expression & Reference for expression patterns \\
\hline D. melanogaster $\mathrm{HSP} 70 \mathrm{Aa}$ & HSP70cA1i & AF295933.1:486-2411 & AAG26887 & Whole body of larvae & Heat & - & Inducible & UniProtKB, ID P82910 \\
\hline D. melanogaster $\mathrm{HSP} 70 \mathrm{Bb}$ & HSP70cA2i & AF295957.1:215-2140 & AAG26911 & Whole body of larvae & Heat & - & Inducible & UniProtKB, ID Q9BIS2 \\
\hline Bombyx HSP70 & HSP70cAli & AB035326.1:102-2024 & BAF69068 & Diapause egg & Heat & Real-time PCR & Inducible & Moribe et al., 2010 \\
\hline Spodoptera HSP70 & HSP70cAli & FJ754276.1:151-2154 & ACN78407 & Fat body & Heat and cold shock & Northern blot & Inducible & Xu et al., 2011 \\
\hline Paralvinella HSP70 form 1 & HSP70cAli & EF580992.1:1-1947 & ABU63808 & Posterior part of the body & Heat & Western blot & $\begin{array}{l}\text { Inducible, but the data may } \\
\text { contain other members }\end{array}$ & Cottin et al., 2008 \\
\hline Lingula HSP70 1B-like & HSP70cA1 & XM_013527000.2:123-2033 & XP_013382454 & - & - & - & - & - \\
\hline A. vaga $\mathrm{HSP} 70-1 \mathrm{~B}$ & HSP70cA2 & -- & GSADVTT00016830001 & - & - & - & - & - \\
\hline A. vaga $\mathrm{HSP} 70-1 \mathrm{~A}$ & HSP70cA1 & - & GSADVT00001811001 & - & - & - & - & - \\
\hline B. ibericus HSP70 & HSP70cAli & GU574486.1:1-1860 & ADR79281 & Whole body & Ultraviolet & Real-time $\mathrm{PCR}$ & Inducible & Kim et al., 2011 \\
\hline Priapulus HSP70 A1-like & HSP70cA1 & XM_014826376.1:219-2171 & XP_014681862 & - & - & - & - & - \\
\hline Aplysia HSP70 B2-1 & HSP70cA1 & XM_005103777.2:352-2253 & XP_005103834 & - & - & - & - & - \\
\hline Aplysia HSP70 B2-2 & HSP70cA2 & XM_005100297.2:191-2110 & XP_005100354 & - & - & - & - & - \\
\hline Crassostrea HSP70 B2 & HSP70cA1 & JH818426.1:39359-41263 & ЕКС 30019 & - & - & - & - & - \\
\hline $\begin{array}{l}\text { Lottia HSP70-4 (hypothetical protein } \\
\text { LOTGIDRAFT_190284) }\end{array}$ & HSP70cA4 & XM_009058212.1:120-2033 & XP_009056460 & - & - & - & - & - \\
\hline $\begin{array}{l}\text { Lottia HSP70-1 (hypothetical protein } \\
\text { LOTGIDRAFT_181897) }\end{array}$ & HSP70cA1 & XM_009053468.1:343-2253 & XP_009051716 & - & - & - & - & - \\
\hline $\begin{array}{l}\text { Lottia HSP70-3 (hypothetical protein } \\
\text { LOTGIDRAFT_209056) }\end{array}$ & HSP70cA3 & XM_009053469.1:216-2129 & XP_009051717 & - & - & - & - & - \\
\hline $\begin{array}{l}\text { Lottia HSP70-2 (hypothetical protein } \\
\text { LOTGIDRAFT_198956) }\end{array}$ & HSP70cA2 & XM_009047345.1:307-2226 & XP_009045593 & - & - & - & - & - \\
\hline $\begin{array}{l}\text { Haliotis heat shock inducible protein } \\
70\end{array}$ & HSP70cA1 & FJ812177.1:169-2079 & АСО 36048 & - & - & - & - & - \\
\hline Asplanchna HSP70 & HSP70cB $1 \mathrm{i}$ & KX119429.1:81-2009 & ARU12813 & Whole body of larvae & $\begin{array}{l}\text { Oxidation stressors } \\
\text { (Paraquat and juglone) }\end{array}$ & Real-time PCR & Inducible & Xu et al., 2017 \\
\hline B. plicatilis $\mathrm{HSP} 70 \mathrm{cB} 1 \mathrm{i}$ & HSP70cBli & AB775784.1:66-2024 & BAO23806 & Whole body of larvae & Heat & $\begin{array}{l}\text { Real-time PCR and in situ } \\
\text { hybridization }\end{array}$ & Inducible & This study \\
\hline B. plicatilis $\mathrm{HSP} 70 \mathrm{CB} 2 \mathrm{i}$ & HSP70cB2i & AB775785.1:65-2035 & BAO23807 & Whole body of larvae & Heat & $\begin{array}{l}\text { Real-time PCR and in situ } \\
\text { hybridization }\end{array}$ & Inducible & This study \\
\hline B. manjavacas $\mathrm{HSP} 70-1$ & HSP70cB1 & - & $\begin{array}{l}\text { BmTi01_isotig01884_1998_175_ } \\
\text { 2172___0 } \\
\text { BmTi01_isotig01885_1998_27_2 } \\
\text { 024_f_2 }\end{array}$ & & - & - & - & - \\
\hline B. manjavacas $\mathrm{HSP} 70-2$ & HSP70сB2 & - & $\begin{array}{l}\text { BmTi01_isotig03034_2025_165_ } \\
\text { 2189___2 }\end{array}$ & - & - & - & - & - \\
\hline B. calyciflorus $\mathrm{HSP} 70-2$ & HSP70cB2 & - & NTPY01000792 & - & - & - & - & - \\
\hline B. calyciflorus $\mathrm{HSP} 70-3$ & HSP70cB3 & - & NTPY01000899 & - & - & - & - & - \\
\hline B. calyciflorus $\mathrm{HSP} 70-1$ & HSP70cB1 & - & NTPY01000620 & - & - & - & - & - \\
\hline A. vaga HSP70-2A & HSP70cB1 & - & GSADVT00032221001 & - & & - & - & - \\
\hline A. vaga $\mathrm{HSP} 70-2 \mathrm{~B}$ & HSP70cB2 & - & GSADVT00050272001 & - & - & - & - & - \\
\hline Ditylenchus HSP70-1 & HSP70cB1 & HQ386232.1:49-1986 & AEP 19214 & - & - & - & - & - \\
\hline C. elegans $\mathrm{HSP} 70 \mathrm{~A}$ & HSP70cBli & $\begin{array}{l}\text { M18540.1:423-627,677-1464,1659- } \\
\text { 2339,2395-2643 }\end{array}$ & AAA28078 & Whole body & Heat & Northern blot & Inducible & Snutch et al., 1988 \\
\hline C. elegans HSP70-1 & HSP70cB2i & NM_070667.4:1-1923 & NP_503068 & Whole body & - & - & Inducible & $\begin{array}{l}\text { WormBase ID: } \\
\text { WBGene00002005 }\end{array}$ \\
\hline Wuchereria HSP70 & HSP70cBli & $\begin{array}{l}\text { AF 167352.1:1973-1069,1451-1555,1731- } \\
2092,2179-2302,2418-2571,2690- \\
2948,3076-3297,3400-3747,3830-\end{array}$ & AAF 32254 & Whole body & Heat & $\begin{array}{l}\text { SDS-PAGE and blotting, } \\
\text { ELISA }\end{array}$ & $\begin{array}{l}\text { Inducible, but the data may } \\
\text { contain other members }\end{array}$ & Ravi et al., 2004 \\
\hline
\end{tabular}




\begin{tabular}{|c|c|c|c|c|c|c|c|c|}
\hline Setaria HSP70 & HSP70cB1c & $\begin{array}{l}\text { AF079360.1:711-807,1003-1107,1212- } \\
\text { 1573,1654-1777,1864-2017,2103- } \\
2361,2450-2671,2772-3119,3198- \\
3384,3499-3578\end{array}$ & AAD13154 & Whole body & Heat & Northern blot & Non-inducible & Jayasena et al., 1999 \\
\hline Strongyloides $\mathrm{HSP} 70$ 1A/1B & HSP70cB1 & XM_024648782.1:1-1935 & XP_024502726 & - & - & - & - & - \\
\hline Schistosoma HSP70-2 & HSP70cB1 & L02415.1:533-2446 & AAA29898 & Whole body & Heat & Northern blot & Non-inducible & Neumann et al., 1993 \\
\hline Limunus HSP cognate 4 & HSP70cB1 & XM_013923214.2:160-2091 & XP_013778668 & 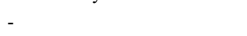 & - & - & - & - \\
\hline Daphnia HSP70Bbb-2 & HSP70cB2 & $\begin{array}{l}\text { LRGB01001774.1:337-903,963- } \\
1285,1342-1597,1655-1858,1928- \\
2025,2086-2194,2252-2641\end{array}$ & gi|1022765301|gb|KZS10545.1| & - & - & - & - & - \\
\hline Priapulus $\mathrm{HSPc}$ & HSP70cB1 & XM_014812613.1:143-2113 & XP_014668099 & - & - & - & - & - \\
\hline Priapulus HSP70 II-like & HSP70cB2 & XM_014817626.1:190-2052 & XP_014673112 & - & - & - & - & - \\
\hline Trichuris HSP70 & HSP70cB1 & $\begin{array}{l}\text { KLL63195.1:c58762-57407,c57345- } \\
\text { 57028,c56967-56683 }\end{array}$ & KFD56098 & - & - & - & - & - \\
\hline Daphnia HSP70Bbb-1 & HSP70cB1 & $\begin{array}{l}\text { LRGB01002121.1:-5532073- } \\
\text { 531866,c531808-531464,c531400-530010 }\end{array}$ & gi|1022763482|gb|KZS09073.1| & - & - & - & - & - \\
\hline Lingula HSP71c & HSP70cB1 & XM_013525464.2:202-2139 & XP_013380918 & - & - & - & - & - \\
\hline M. nipponense HSP70-1 & HSP70cB1i & KC460343.1:253-2202 & AGM50430 & Gill & Heat & Real-time PCR & Inducible & Xiu et al., 2014 \\
\hline M. nipponense HSC70 & HSP70cB2i & DQ660140.1:99-2048 & ABG45886 & Gill & Heat & Real-time PCR & Inducible & Xiu et al., 2014 \\
\hline Crassostrea HSP71c & HSP70cB1 & AB122064.1:33-2012 & BAD15287 & - & - & - & - & - \\
\hline Mytilus $\mathrm{HSP} 70$ & HSP70cB1i & AY861684.1:71-2035 & AAW52766 & Whole body & Heat, heat-killed bacteria & Real-time PCR & Inducible & Cellura et al., 2006 \\
\hline Mytilus HSP71c & HSP70cB2 & $\begin{array}{l}\text { AJ783714.1:475-682,1087-1292,1752- } \\
1904,2462-3017,5566-5967,6815-7254\end{array}$ & СAH04109 & - & - & - & - & - \\
\hline $\begin{array}{l}\text { Helobdella hypothetical protein } 2 \\
\text { HELRODRAFT }\end{array}$ & HSP70cB1 & XM_009023904.1:1-1878 & XP_009022152 & - & - & - & - & - \\
\hline $\begin{array}{l}\text { Helobdella hypothetical protein } \\
\text { HELRODRAFT }\end{array}$ & HSP70cB2 & XM_009031197.1:188-2125 & XP_009029445 & - & - & - & - & - \\
\hline Tevnia HSP70-1 & HSP70cB1 & FN860147.1:1-411,757-2100,2515-2709 & CBM42052 & - & - & - & - & - \\
\hline Tevnia HSP70-2 & HSP70cB2 & FN860148.1:1-411,865-2208,2623-2817 & CBM42053 & - & - & - & - & - \\
\hline Perinereis HSP70-1 & HSP70cB1i & KU255783.1:49-2019 & AND99892 & Whole body & $\mathrm{Cu}^{2+}$ & Real-time PCR & Inducible & Zhang et al., 2016 \\
\hline Perinereis HSP70-2 & HSP70cB2 & HQ449186.1:89-2050 & ADR66514 & - & - & - & - & - \\
\hline Paralvinella HSP70 form 2 & HSP70cB $1 \mathrm{i}$ & EF580993.1:1-1962 & ABU63809 & Posterior part of the body & Heat & Western blot & $\begin{array}{l}\text { Inducible, but the data may } \\
\text { contain other members }\end{array}$ & Cottin et al., 2008 \\
\hline Haliotis $\mathrm{HSC70}$ & HSP70cB1 & FJ812176.1:77-2032 & ACO36047 & - & - & - & - & - \\
\hline Aplysia $\mathrm{HSC71}$ & HSP70cB1 & XM_005097955.2:103-2067 & XP_005098012 & - & - & - & - & - \\
\hline $\begin{array}{l}\text { Lottia HSC70 (hypothetical protein } \\
\text { LOTGIDRAFT_177837) }\end{array}$ & HSP70cB1 & XM_009048116.1:1-1962 & XP_009046364 & - & - & - & - & - \\
\hline Octopus $\mathrm{HSC71}^{-1}$ & HSP70cB1 & XM_014927588.1:112-2067 & XP_014783074 & - & - & - & - & - \\
\hline Octopus HSP71-like & HSP70cB2 & XM_-014929288.1:100-2058 & XP_014784774 & - & - & - & - & - \\
\hline Dugesia HSP70 & HSP70cB1 & EU380241.1:53-1999 & ABY83101 & Whole body & $\begin{array}{l}\text { Heat, amputation and } \\
\text { starvation }\end{array}$ & Semi-quantitative RT-PCR & Inducible & Ma et al., 2009 \\
\hline Oncorhynchus HSC71 & HSP70cB1c & $\begin{array}{l}\text { S85730.1:27774-2978,32222-3427,3528- } \\
3680,3904-4459,4686-4888,5108- \\
5306,5536-5768,5965-6165\end{array}$ & AAB21658 & Gonad cell line & Heat & Northern blot & Non-inducible & Zafarullah et al., 1992 \\
\hline X. maculatus $\mathrm{HSC70}$ & $\mathrm{HSP} 70 \mathrm{cB} 1 \mathrm{c}$ & АВ062115.1:135-2048 & BAB72169 & Muscle and liver & Heat & Northern blot & Non-inducible & Yamashita et al., 2004 \\
\hline H. sapiens HSC71 (HSPA8) & HSP70cB6c & NM_006597.6:79-2019 & NP_006588 & - & - & - & - & - \\
\hline Rattus $\mathrm{HSC} 71$ & HSP70cBlc & NM_024351.2:81-2021 & NP_077327 & Brain & Axonal synapsin & $\begin{array}{l}\text { Two-color superresolution } \\
\text { microscopy }\end{array}$ & Non-inducible & Ganguly et al., 2017 \\
\hline Bos HSC71 & HSP70cB1 & NM_174345.4:76-2028 & P19120 & - & - & - & - & - \\
\hline Pelodiscus $\mathrm{HSC70}$ & HSP70cB1i & HQ219723.1:81-2021 & ADO17794 & $\begin{array}{l}\text { Liver, lung, heart, and skeletal } \\
\text { muscle }\end{array}$ & Heat & Western blot, Real-time PCR & Inducible & Li et al., 2012 \\
\hline Bos HSP70 & HSP70cB2i & U09861.1:157-2082 & AAA73914 & Skeletal muscle & Bacteria & $\begin{array}{l}\text { Two-dimensional gel } \\
\text { electrophoresis }\end{array}$ & Inducible & Gutierrez and Guerriero, 1995 \\
\hline
\end{tabular}




\begin{tabular}{|c|c|c|c|c|c|c|c|c|}
\hline H. sapiens HSP70-1B (HSPA1B) & HSP70cB2i & NM_005346.5:214-2139 & NP_005337 & - & - & - & Inducible & Uniprot P0DMV8 \\
\hline Rattus HSP70 & HSP70cB2i & NM_031971.2:1248-3173 & NP_114177 & Pancreas & Heat & Western blot & Inducible & Lee et al., 2018 \\
\hline H. sapiens HSP70-likel (HSPA-1L) & HSP70cB3c & NM_005527.4:409-2334 & NP_005518 & HeLa cells & Heat & Northern blot & Non-inducible & $\begin{array}{l}\text { Uniprot P34931, Milner and Campbell, } \\
1990\end{array}$ \\
\hline H. sapiens HSP70-6 (HSPA6) & HSP70cB5i & NM_002155.5:120-2051 & NP_002146 & Neuronal cells & Aarimoclomol & Immunostaining, Western blot & Inducible & $\begin{array}{l}\text { Deane and Brown, 2018, UniProt } \\
\text { P17066 }\end{array}$ \\
\hline X. maculatus HSP70-1 & HSP70cB1i & АВ062113.1:83-1999 & BAB72167 & Muscle and liver & Heat & Northern blot & Inducible & Yamashita et al., 2004 \\
\hline X. maculatus $\mathrm{HSP} 70-2$ & HSP70cB2i & АВ062114.1:131-2050 & BAB72168 & Muscle and liver & Heat & Northern blot & Inducible & Yamashita et al., 2004 \\
\hline H. sapiens HSP70-like2 (HSPA2) & HSP70cB4c & NM_021979.3:383-2302 & NP_068814 & Lung carcinoma cell & Heat & Immunostaining, Western blot & Non-inducible & Ścieglińska et al., 2008 \\
\hline Alligator $\mathrm{HSP} 70 \mathrm{~A}$ & HSP70cB1 & AB306279.1:43-1962 & BAF94142 & - & - & - & - & \\
\hline S. cerevisiae SSA1 & - & NC_001133.9:c141431-139503 & YAL005C & - & Heat & - & Inducible & Boorstein et al., 1994 \\
\hline S. cerevisiae SSA2 & - & NC_001144.5:c97485-95566 & YLL024C & - & Heat & - & Non-inducible & Boorstein et al., 1994 \\
\hline S. cerevisiae SSA3 & - & NC_001134.8:c86448-84499 & YBL075C & - & Heat & - & Inducible & Boorstein et al., 1994 \\
\hline S. cerevisiae SSA4 & - & NC_001137.3:364589-366517 & YER103W & - & Heat & - & Inducible & Boorstein et al., 1994 \\
\hline C. elegans $\mathrm{HSP} 70 \mathrm{~F}$ & HSP70mA1 & NM_071890.4:1-1974 & NP_504291 & - & - & - & - & - \\
\hline H. sapiens HSP70m (HSPA9) & HSP70mAli & NM_004134.7:84-2123 & NP_004125 & Cancer cells & Ovarian carcinoma & Immunostaining, Western blot & Inducible & Xu et al., 2019 \\
\hline Pongo stress-70 protein & HSP70mA1 & NM_001133388.1:101-2140 & NP_001126860 & - & - & - & - & - \\
\hline Cricetulus stress-70 protein & & NM_001246829.1:1-2040 & NP_001233758 & - & - & - & - & - \\
\hline A. vaga $\mathrm{HSP} 70 \mathrm{mA2}$ & HSP70mA2 & - & GSADVT00049157001 & - & - & - & - & - \\
\hline A. vaga $\mathrm{HSP} 70 \mathrm{~mA} 1$ & HSP70mA1 & - & GSADVT00003702001 & - & - & - & - & - \\
\hline Daphnia HSC70-5 & HSP70mA1 & $\begin{array}{l}\text { LRGB01000716.1:c34677-3389,c3326- } \\
3203,, 3078-2880, \text { c2819-2228,c2144-1297 }\end{array}$ & gi|1022772237|gb|KZS16423.1| & - & - & - & - & - \\
\hline Scerevisiae ECM10 & - & NC_001 137.3:94644-96578 & YEL030W & - & - & - & - & - \\
\hline Scerevisiae SSC1 & - & NC_001142.9:c521602-519638 & YJR045C & - & - & - & - & - \\
\hline C. elegans BiP, heat shock protein 3 & HSP70erAlc & $\begin{array}{l}\text { M26604.1:442-628,675-1148,1387- } \\
2132,2237-2815\end{array}$ & AAA28074 & Whole body of larvae & Heat & Not mentioned & Non-inducible & Heschl and Baillie, 1989 \\
\hline Crassostrea GRP & HSP70erAli & AB122065.1:108-2093 & BAD15288 & Gill and adductor muscle & Heat & Northern blot & Inducible & Yokoyama et al., 2006 \\
\hline B. ibericus $\mathrm{HSC70}$ & HSP70erAli & GU574487.1:1-1962 & ADR79282 & Whole body & Ultraviolet & Real-time PCR & Inducible & Kim et al., 2011 \\
\hline B. calyciflorus $\mathrm{HSC} 70-2$ & HSP70erA2 & NTPY01000000 & NTPY01001024 & - & - & - & - & - \\
\hline B. calyciflorus $\mathrm{HSC} 70-1$ & HSP70erA1 & NTPY01000000 & NTPY01001024 & - & - & - & - & - \\
\hline B. manjavacas $\mathrm{HSC} 71$ & HSP70erA1 & - & $\begin{array}{l}\text { 1977_126_2102_r_0 } 01 \\
\text { BmTi } 01 \text { _sotig03139 }\end{array}$ & - & - & - & - & - \\
\hline A. vaga HSP70erA2 & HSP70erA2 & - & GSADVT00030804001 & - & - & - & - & - \\
\hline A. vaga HSP70erA1 & HSP70erAl & - & GSADVT00008959001 & - & - & - & - & - \\
\hline Daphnia HSC70-4 & HSP70erA1 & $\begin{array}{l}\text { LRGB01000024.1:1105780- } \\
1105910,1105969-1106200,1106260- \\
1106476,1106539-1106876,1106942- \\
1107467,1107528-1108063\end{array}$ & gi|1022777923|gb|KZS21417.1| & - & - & - & - & - \\
\hline H. sapiens Bip & HSP70erAli & NM_005347.5:218-2182 & NP_005338 & $\begin{array}{l}\text { Umbilical vein endothelial } \\
\text { cells }\end{array}$ & $\begin{array}{l}\text { Thapsigargin } \\
\text { (endoplasmic stress) }\end{array}$ & Western blot & Inducible & $\begin{array}{l}\text { Uniprot P1 1021, Milner and Campbell, } \\
1990\end{array}$ \\
\hline Schistosoma HSP70-1 & HSP70erA1 & XM_018794726.1:55-2001 & XP_018649109 & - & & - & - & \\
\hline S. cerevisiae KAR2 & - & NC_001142.9:381327-383375 & YJL034W & - & Heat & - & Inducible & Boorstein et al., 1994 \\
\hline
\end{tabular}


Bp HSP 70-1

Bp_HSP70-2

Bi HSC70

Bi-HSP70

Ab HSP70

Pr_HSP70

BP_HSP 70-1 Bp_HSP70-2 Bi_HSC70 Bi_HSP70 Ab HSP70 Pr ${ }^{-}$HSP70

BP_HSP7 0-1 BP_HSP70-2 Bi_HSC70 Bi_HSP70 Ab_HSP70 Pr_HSP70

BP_HSP70-1 Bp_HSP70-2 Bi_HSC70 Bi_HSP70 Ab_HSP70 Pr HSP7O

BP_HSP 70-1 BP_HSP70-2 Bi_HSC70 Bi_HSP70 Ab_HSP70 Pr_HSP70

BP_HSP 70-1 Bp_HSP70-2 Bi HSC70 $\mathrm{Bi}$ HSP70 Ab_HSP70 Pr_HSP70

BP_HSP70-1 BP_HSP70-2 Bi_HSC70 Bi HSP70 Ab_HSP70 Pr_HSP70

BP_HSP70-1 Bp_HSP70-2 Bi_HSC70 Bi HSP70 Ab_HSP70 Pr_HSP70

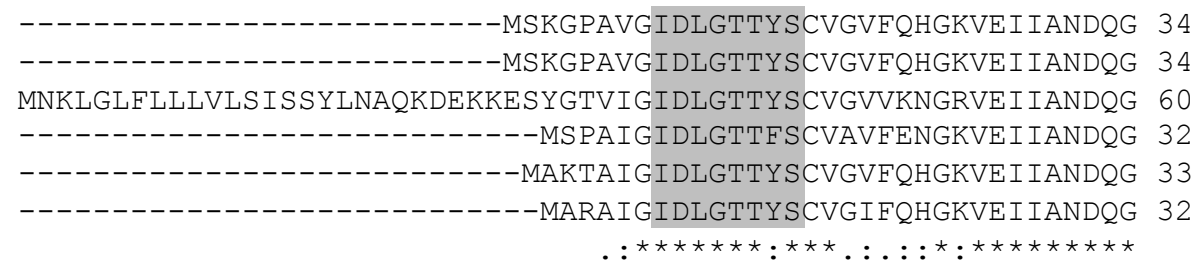

NRTTPSYVAFT-DTERLIGDAAKNQVAMNPNNTVFDAKRLIGRKFDDLTVQADMKHWPFT 93 NRTTPSYVAFT-DTERLIGDAAKNQVAMNPNNTVFDAKRLIGRKFDDPTVQADMKHWPFT 93 NRITPSYVAFTSDGERLIGDAAKNQLTSNPENTIFDAKRLIGREFKDSSVQGDMKYWPFK 120 NRTTPSYVAFT-DTERI IGDAAKAQVATNPTNTVFDAKRLIGRRFDEHTVQSDMKHWPFK 91 NRTTPSYVAFT-DTERLIGDAAKNQVAMNPTNTVFDAKRLIGRRFDDSTVQSDMKHWPFT 92 NRTTPSYVAFT-DSERLIGDAAKNQVAMNPNNTIFDAKRLIGRKFDDATVQADMKHWPFK 91

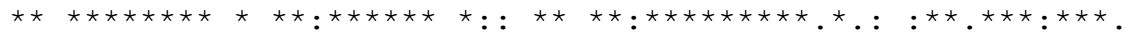

VISDSGRPKIQVEFKGETKSFYPEEVSSMVLTKMKETAEAYLGKKVTDAVVTVPAYFNDS 153 VISDSGRPKIQVEFKGETKSFYPEEVSSMVLTKMKETAEAYLGKKVTDAVVTVPAYFNDS 153 VVDKNNKPHIKVKSADEEKLFAAEEVSAMVLGKMKEIAEAYLGKPVTHAVVTVPAYFNDA 180 LVNVNGKPKIQVEFKNSSKTFSPEEISSMVLTKMKETAESYLRKKSKKCSHHSTGLFQRF 151 VVNEGGKPKIQVEFKGEKKTFFPEEISSMVLIKMKEIAEAYLGKKVNDAVITVPAYFNDS 152 VISEGGKPKIQVEYKNETKSFTPEEVSSMVLTKMKEIAEAYLGKKISEAVITVPAYFNDS 151

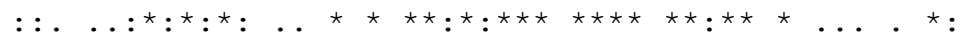

QRQATKDAGAIAGLNVLRI INEPTAAAIAYGLDKKGGGEKNILIFDLGGGTFDVSILTIE 213 QRQATKDAGAIAGLNVLRI INEPTAAAIAYGLDKKGGGEKNILIFDLGGGTFDVSILTIE 213 QRQATKDAGTIAGMTVMRI INEPTAAVYCLRLGQK-EGEKNILVFDLGGGTFDVSLLTID 239 ATTSYQRCWINSRFKCATYYQRAYCCCSCLWLRKKFKRRKNVLIYDLGGGTFDVSVLSIN 211 QRQATKDAGAISGLNVLRI INEPTAAAIAYGLDKKVGGEKNVLIFDLGGGTFDVSILTIE 212 QRQATKDAGVIAGLNVLRI INEPTAAAIAYGLDKKVTGERNILIFDLGGGTFDVSVLKIE 211

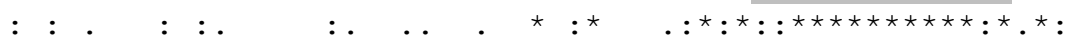

EGIFEVKSTAGDTHLGGEDFDNRLVNHFVEEFKRKNKKDITSNKRALRRLRTACERAKRT 273 EGIFEVKSTAGDTHLGGEDFDNRLVNHFVEEFKRKNKKDITSNKRALRRLRTACERAKRT 273 NGVFEVVATNGDTHLGGEDFDNRVMEHFIKLFKKKTGKDIRKDNRAVQKLRREVEKAKRT 299 DGLFEVLSTNGNTHLGGEDFDNNLVNFFASEFRRKHGKDLSRNQKALRRLRTACERAKRT 271 EGIFEVKSTAGDTHLGGEDFDNRLVNHFVEEFKRKHKKDITGNKRAIRRLRTACERAKRT 272 EGIFEVKSTAGDTHLGGEDFDNRMVSHFVQEFKRKNNKDLSQNKRGLRRLRTACERAKRT 271

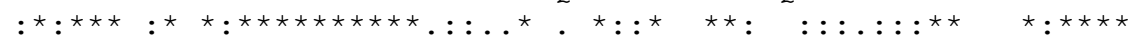

LSSSAQANIEIDSLHEGVDFYTSITRARFEELCADLFRGTLDPVEKALRDAKMDKSSVNE 333 LSSSAQANIEIDSLHEGVDFYTSITRARFEELCADLFRGTLDPVEKALRDAKMDKSSVNE 333 LSTQFDTRIEIESFFDGEDFSETFTRARFEELNMDLFRNTLKPVQKVLEDAGLKKTEIDE 359 LSSSAEATIEVDSLYEGTDFNSKISRARFEELNMDLFRSTLHLVEQALKDARITKTKIDE 331 LSSSAQANIEIDSLHEGVDFYTNITRARFEELNADLFRGCLDPVEKSLRDAKLDKAQIHE 332 LSSSSQASIEIDSLHEGIDFYSTITRARFEELCADLFRSTLEPVEKALRDAKMDKASIHE 331

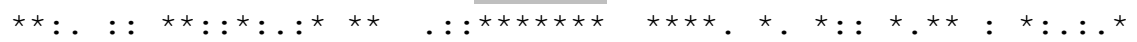

IVLVGGSTRIPKVQKLLQDFFNGKELNKS INPDEAVAYGAAVQAAILTGDKSEAVQDLLL 393 IVLVGGSTRIPKVQKLLQDFFNGKELNKS INPDEAVAYGAAVQAAILTGDKSEAVQDLLL 393 IVLVGGSTRIPKIQQLVKEYFDGKEPSRGINPDEAVAYGAAVQGGVLSGEDVG--GEVVL 417 IVLVGGSTRIPRIQKAFRGFFNGKQLNKSINPDEAVAYGAAVQAAILSGDKSREIKDVLL 391 IVLVGGSTRIPKVQKLLQDFFNGKELNKS INPDEAVAYGAAVQAAILTGDKSEAVQDLLL 392 IVLVGGSTRI PKVQKLLQDFFNGKELNKS INPDEAVAYGAAVQAAILTGDKSEEVKDVLL 391

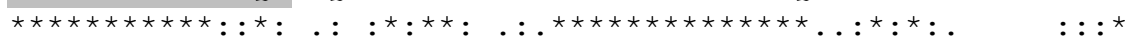

LDVAPLSLGIETAGGVMTALIKRNTTIPTKQTQTFTTYADNQPGVLIQVYEGERAMTKDN 453 LDVAPLSLGIETAGGVMTALIKRNTTIPTKQTQTFTTYADNQPGVLIQVYEGERAMTKDN 453 LDVNPLTMGIETVGGVMTKLI PRNTVIPTKNP-KFSTASDNQPTVTIAVYEGERPMTKDN 476 VDVAPLSLGIETAGGVMTNLINRNSRI PVKTSQIFTTYSDNQTAVSIRVFEGERALVQDN 451 LDVAPLSLGIETAGGVMTSLIKRNTTIPTKQTQVFTTYSDNQPGVLIQVYEGERAMTKDN 452 LDVAPLSLGIETAGGVMTPLIKRNTTIPTKQTQTFTTYSDNQPGVDIKVFEGERSMTRDN 451

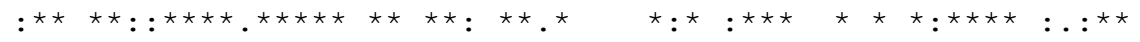




\begin{abstract}
BP_HSP70-1 BP_HSP70-2

$\mathrm{Bi} \mathrm{HSC} 70$

Bi-HSP70

$\mathrm{Ab}$ HSP70

Pr_HSP70

HLLGKFELSGIPPAPRGVPQIEVTFDIDANGILNVSAADKSTGKTNKITITNDKGRLSKE 513 HLLGKFELSGIPPAPRGVPQIEVTFDIDANGILNVSAADKSTGKTNKITITNDKGRLSKE 513 HLLGKFDLTGIPPAPRGVPQIEVTFEIDANG I LKVSAEDKGTGNKNNIVINNNQNRLSPE 536 HLLGNFDLVGI PPAPRGIPQIDVTFDIDANG I LSVSAKENSSGKSNNITIKNDKGRLSKE 511 NLLGKFELTGIPPAPRGVPQIEVTFDIDANGILNVSACDKSTGKQNKITITNDKGRLSKE 512 HLLGNFELSGIPPAPRGVPQIEVTFDIDANGILNVTAVDKSTGRENKITITNDKGRLSKD 511

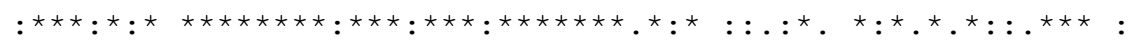

BP_HSP70-1

BP HSP70-2

Bi HSC70

Bi_HSP70

Ab HSP 70

Pr_HSP70

BP_HSP70-1

BP HSP70-2

Bi_HSC70

Bi_HSP70

Ab_HSP70

Pr_HSP70

BP_HSP7 0-1

Bp_HSP70-2

Bi HSC70

Bi HSP70

Ab_HSP70

Pr_HSP70

EIDRMVNEAEKYKKDDEEQRDKVAAKNSLESYCFNMKQTVED-EKLAAKISADDKKKILD 572 DIDRMVNEAEKYKKDDEEQRDKVAAKNSLESYCFNMKQTVED-EKLAAKISADDKKKILD 572 EIERMIKDAEKFADEDKKVKEKVEAKNELESYVYSLKNQLNDKEKLGNKLSEDEKETINS 596 EIERMINEAERFRAEDDRQRDRIASKNKLETYIFAVKQALDD-AK---NLSSSDKKVCQE 567 DIERMVNDAEKYKKDDEQQRERITAKNSLESYCFNMKQTIED-EKLAAKVSADEKKKILD 571 QIEQMVAEAEKYKKEDEAQRDRISAKNSLESYCFNMKTSIND-DKIGAKISADDKAKITE 570

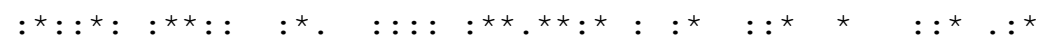

ACEEALKWLDSNQTAEKDEFEHKMKEVEKICSPI ITQLYQGAGGAPGGMPGGM----PGG 628 ACEEALKWLDSNQTAEKDEFEHKMKEVEKICSPIITQLYQGAGGAPGGMPGGMPGGMPGG 632 AVDEKIKWLESNSDAEVDDFKEQKKALEEIVNPIMTKLYQQNSENAGSSDSQTNDEL--- 653 ACRKELKWLEANQMADKDEFAFHYKELSRKCMPLMKKIHSGERNGPTVEEVE-------- 619 ACENALKWLDANQTAEKDEFEHKLKEIEKTCSPIITKLYQG--GAPGGMPGGMPGGAGA- 628 TIESALKWMETNQLAEKDEFEHKLKEVEKICSPIMTKLYGGEAGGHAG-PGGHAGGA--- 626 $: \quad . \quad{ }^{\star}:::{ }^{\star} \cdot{ }^{\star}:{ }^{\star}:{ }^{\star}:{ }^{\star}: \ldots \quad{ }^{\star}::$ : : :

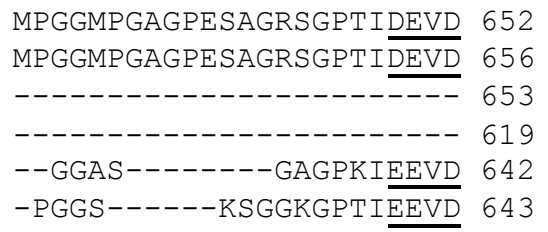

Supplementary fig. S1. Comparison of the deduced amino acid sequences of cDNAs encoding HSP70-1 and HSP70-2 of Brachionus plicatilis with those of the HSP70 family members from other rotifers. Dashes denote gaps introduced to maximize homology. The HSP70 protein family signatures are meshed, and the EEVD motifs are boxed. The non-organelle consensus motifs and bipartite nuclear localization motifs are shown in blue and red letters, respectively. The numbers in the right margin of the sequences represent residues from the $\mathrm{N}$-terminus. $\mathrm{Bi}$, B. ibericus (ADR79281, ADR79282); Ab, Asplanchna brightwelli (ARU12813). 


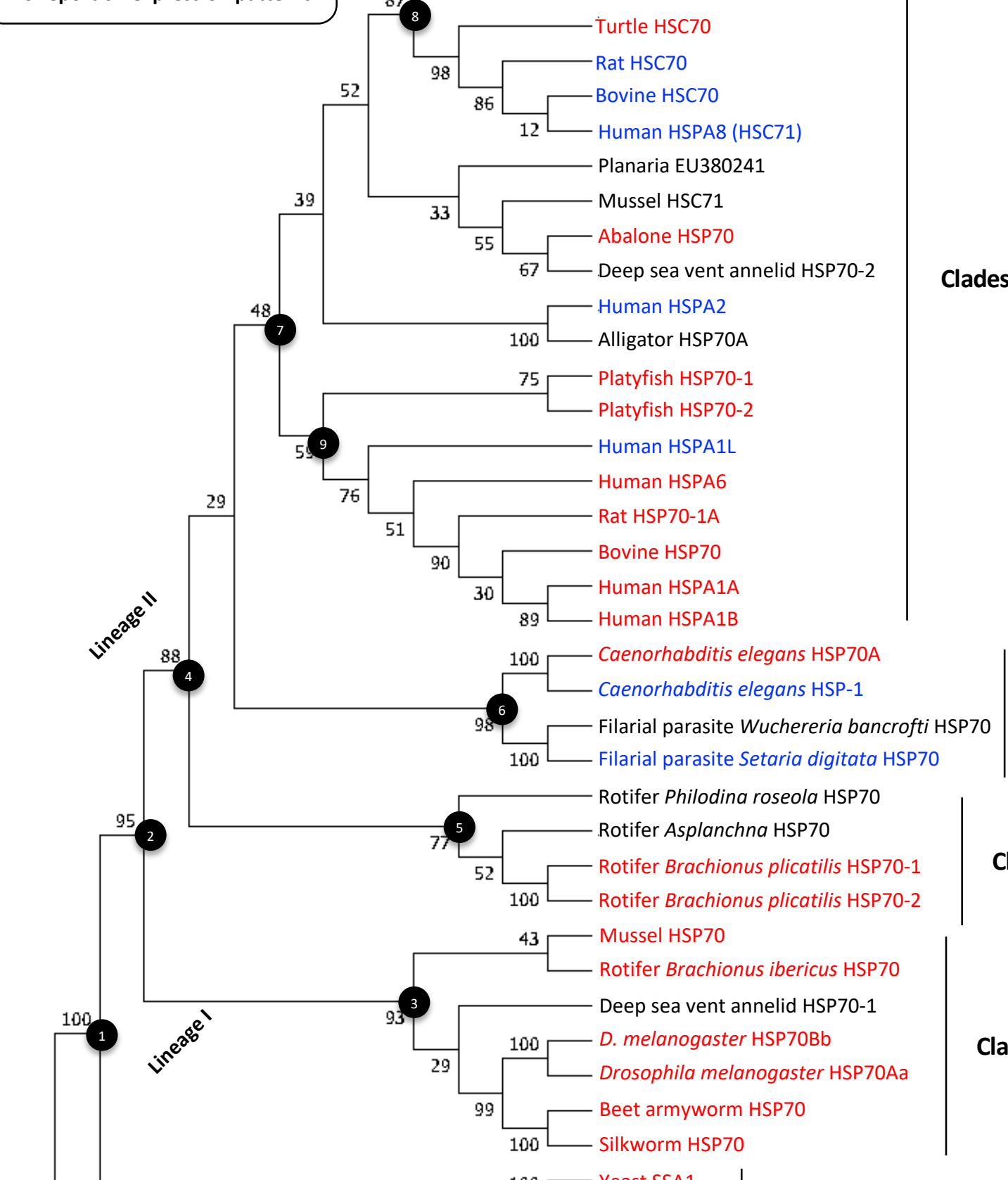

Clade 3

Clade 2

Clade 1

\section{Clade 6}

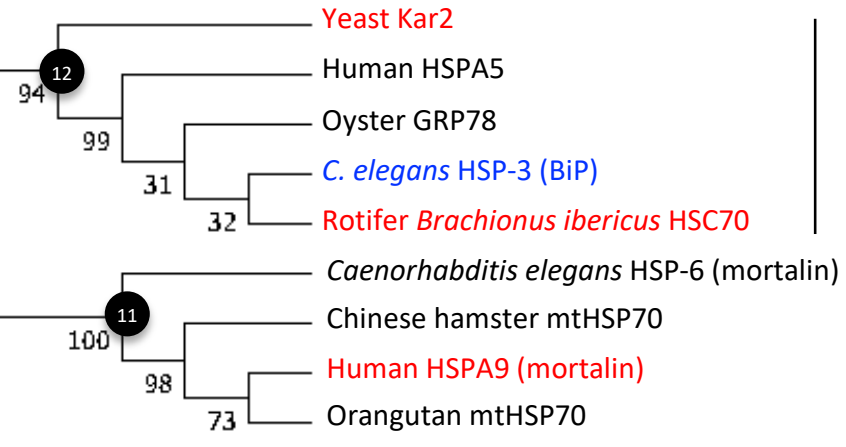

Endoplasmic reticulum 
Supplementary fig. S2. Maximum likelihood tree of HSP70 family members. A bootstrap consensus tree was constructed by the maximum likelihood method using the $L G+G$ model. The bootstrap values from a 1000-replicate analysis are given at the nodes in percentage. Nodes and clades in fig. 2 are indicated in the tree. Stress-inducible and constitutive genes are shown in red and blue, respectively. No data on the stress inducibility is available for genes shown in black. 


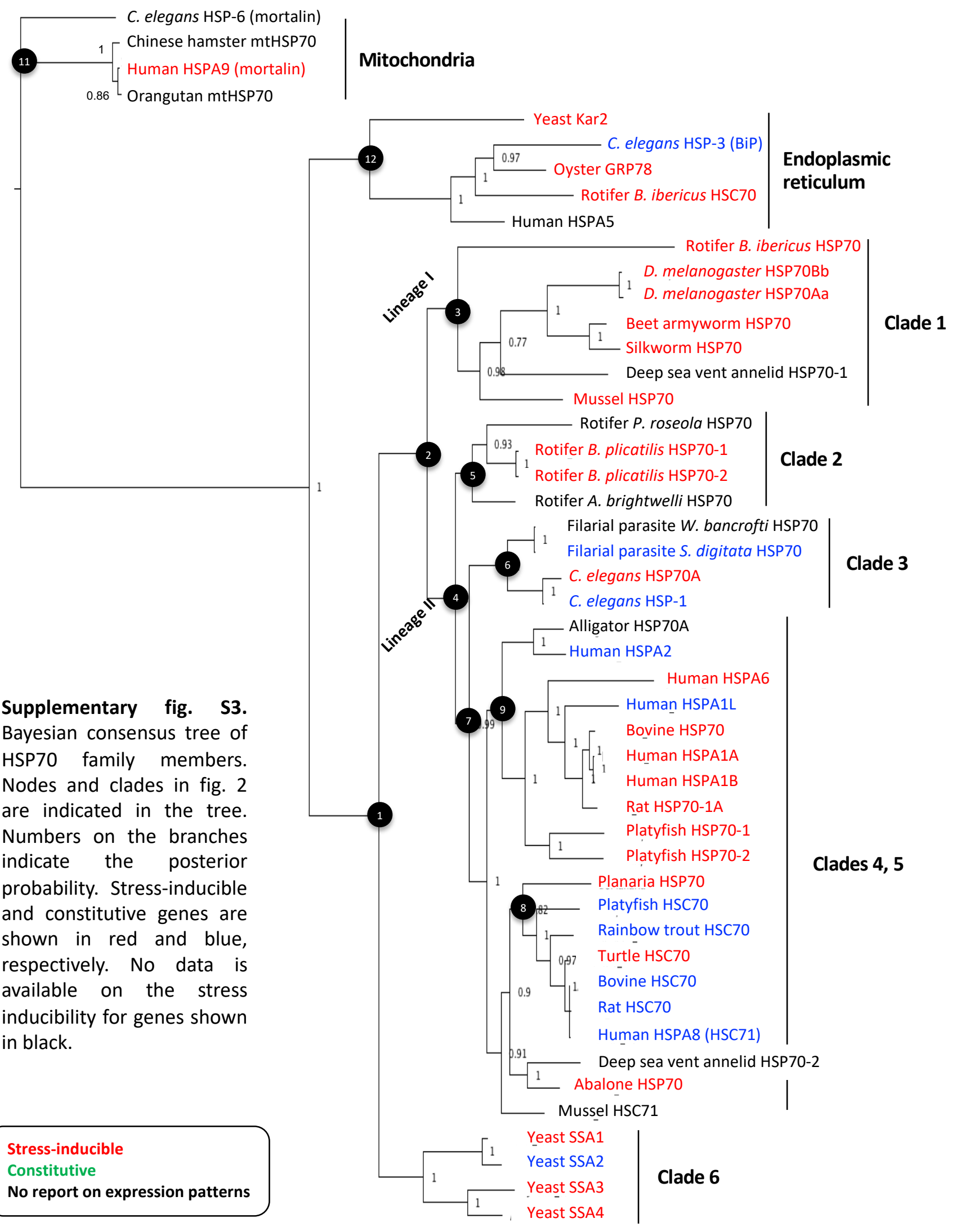


Supplementary fig. S3. Bayesian consensus tree of HSP70 family members. Nodes and clades in fig. 2 are indicated in the tree. Numbers on the branches indicate the posterior probability. Stress-inducible and constitutive genes are shown in red and blue, respectively. No data is available on the stress inducibility for genes shown in black. 
bioRxiv preprint doi: https://doi.org/10.1101/2020.09.21.307264; this version posted April 22, 2021. The copyright holder for this preprint (which was not certified by peer review) is the author/funder, who has granted bioRxiv a license to display the preprint in perpetuity. It is made available under aCC-BY-NC-ND 4.0 International license.

Aplysia HSP70-2

Haliotis HSP70

Lottia_HS̄P $70-4$

Bplicatilis HSP70-1

Avaga_HSP70ёrA

Bibericus HSC70

BCHSC70-2

Celegans HSP70F

Daphnia_HSP70c5-1

Pongo HS̄P70m

Hsapiēns_HSP70m

Aplysia HSP70-2

Haliotis HSP70

Lottia HS $\mathrm{S} 70-4$

Bplicatilis HSP70-1

Avaga_HSP70ēerA

Biberícus HSC70

BCHSC70-2

Celegans HSP70F

Daphnia_HSP70c5-1

Pongo HS̄P70m

Hsapiens_HSP70m

Aplysia_HSP70-2

Haliotis HSP70

Lottia_HS̄P70-4

Bplicatilis HSP70-1

Avaga HSP70ēerA

Bibericus HSC70

BCHSC70-2

Celegans HSP7OF

Daphnia HSP70c5-1

Pongo_HS̄P70m

Hsapiens_HSP70m

Aplysia_HSP70-2

Haliotis HSP7O

Lottia_HS̄P70-4

Bplicatilis_HSP70-1

Avaga_HSP70erA

Bibericus HSC70

BCHSC70-2

Celegans HSP70F

Daphnia HSP70c5-1

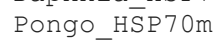

Hsapiens_HSP70m

Aplysia_HSP70-2

Haliotis HSP7O

Lottia HSP 70-4

Bplicatilis_HSP70-1

Avaga HSP70erA

Bibericus_HSC70

BCHSC70-2

Celegans_HSP70F

Daphnia HSP70c5-1

Pongo_HS̄P70m

Hsapiens_HSP70m

Aplysia HSP70-2

Haliotis HSP7O

Lottia HSP70-4

Bplicatilis_HSP70-1

Avaga HSP70ēA

Bibericus_HSC70

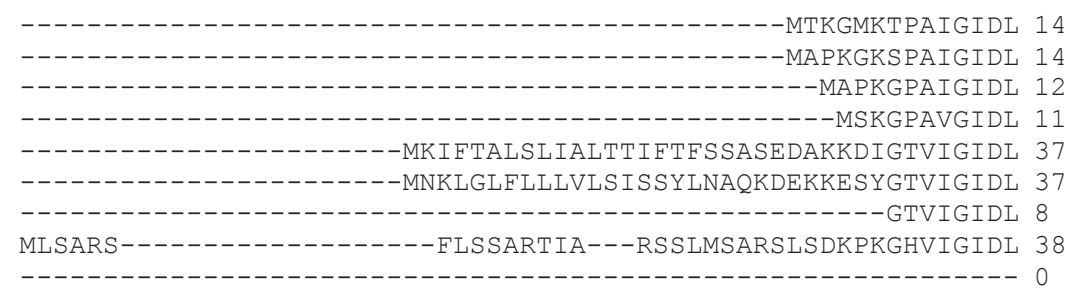

MISASRAVAARLVGAAASRGPTAARYQDGWNGLSHEAFRIVSRRDYASEAIKGAVVGIDL 60 MISASRAAAARLVGAAASRGPTAARHQDSWNGLSHEAFRLVSRRDYASEAIKGAVVGIDL 60

GTTYSCVGIFQHGKVEIIANDQGNRTTPSYVAFT-DTERLVGDAAKNQAALNPSNTIFDA 73 GTTYSCVGVFQNGQVEI IANDQGNRTTPSYVAFT-DTERLIGDAAKNQVALNPQNTVFDA 73 GTTFSCVGVFQHGAVEI IANDQGNRTTPSYVAFT-DSERLIGDSAKNQVALNPSNTIFDA 71 GTTYSCVGVFQHGKVEI IANDQGNRTTPSYVAFT-DTERLIGDAAKNQVAMNPNNTVFDA 70 GTTYSCVGIFKNGRVEI IANDQGNRITPSYVAFTAEGERLIGDAAKNQLTSNPENTVFDA 97 GTTYSCVGVVKNGRVEI IANDQGNRITPSYVAFTSDGERLIGDAAKNQLTSNPENTIFDA 97 GTTYSCVGVVKNGRVEI IANDQGNRITPSYVAFTSEGERLIGDAAKNQLTSNPENTIFDA 68 GTTNSCVS IMEGKTPKVIENAEGVRTTPSTVAFTADGERLVGAPAKRQAVTNSANTLFAT 98 -------MEGQTPKVIENAEGSRTTPSVIAFTKDGERLAGMPAKRQAVTNAQNTLYAT 51 GTTNSCVAVMEGKQAKVLENAEGARTTPSVVAFTADGERLVGMPAKRQAVTNPNNTFYAT 120 GTTNSCVAVMEGKQAKVLENAEGARTTPSVVAFTADGERLVGMPAKRQAVTNPNNTFYAT 120

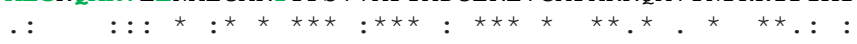

KRLIGRKFTDKSVQSDMKHWPFQVAE-VDSRPKIEAEYKGERKLFAPEEVSSMVLSKMKE 132 KRLIGRQFDDSAVQKDMKHWPFRVVNSSGSKPKLQVEYKGEKKTFAPEEISSMVLNKMKE 133 KRLIGRKFDDATIQSDMKHWPFKVVK-DGDKPKLQAEFKNELKTFSPEEVSAMVLTKMKE 130 KRLIGRKFDDLTVQADMKHWPFTVIS-DSGRPKIQVEFKGETKSFYPEEVSSMVLTKMKE 129 KRLIGREFSDSSVQQDMKHFPFKVVE-KNSKPAIQISTGKEQKLFTPEEISAMVLGKMRE 156 KRLIGREFKDSSVOGDMKYWPFKVVD-KNNKPHIKVKSADEEKLFAAEEVSAMVLGKMKE 156 KRLIGREFSDPSVQGDMKYWPFKVVE-KNKKPYIKVKSGDQDKLFAPEEISAMVLGKMKE 127 KRLIGRRYEDPEVQKDLKVVPYKIVKASNGDAWVE----AQGKVYSPSQVGAFVLMKMKE 154 KRLIGRKFEDPEVKKDMKTVSYKIVRASNGDAWVE----AQGKLYSPSQIGAFVLTKMKE 107 KRLIGRRYDDPEVQKDIKNVPFKIVRASNGDAWVE----AHGKLYSPSQIGAFVLMKMKE 176 KRLIGRRYDDPEVQKDIKNVPFKIVRASNGDAWVE----AHGKLYSPSQIGAFVLMKMKE 176

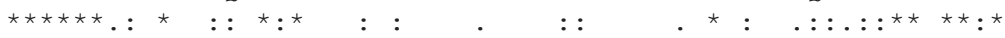

TAEAYLGQKVTEAVITVPAYFNDSQRQATKDAGAIAGLNVLRMINEPTAAALAYGLDKGQ 192 TAEAYLGQQVTDAVITVPAYFNDSQRQATKDAGAIAGLNVLRI INEPTAAALAYGLDKNL 193 TAEAYLGENVTNAVITVPAYFTDSQRSATKDAGVIAGLNVLRI INEPTAAALAYGLDKKT 190 TAEAYLGKKVTDAVVTVPAYFNDSQRQATKDAGAIAGLNVLRI INEPTAAAIAYGLDKKG 189 IAEAYLGKKVTHAVVTVPAYFNDAQRQATKDAGTISGLNVLRI INEPTAAAIAYGLDKK- 215 IAEAYLGKPVTHAVVTVPAYFNDAQRQATKDAGTIAGMTVMRI INEPTAAVYCLRLGQK- 215 IAEAYLGKNVTHAVVTVPAYFNDAQRQATKDAGTISGMTVMRI INEPTAAAIAYGLDKK - 186 TAESYLGTTVNNAVVTVPAYFNDSQRQATKDAGQISGLNVLRVINEPTAAALAYGLDKD- 213 TAEAYLGTPVKNAVVTVPAYFNDSQRQATKDAGQISGLNVLRVINEPTAAALAYGMDKS - 166 TAENYLGHTAKNAVITVPAYFNDSQRQATKDAGQISGLNVLRVINEPTAAALAYGLDKS - 235 TAENYLGHTAKNAVITVPAYFNDSQRQATKDAGQISGLNVLRVINEPTAAALAYGLDKS - 235

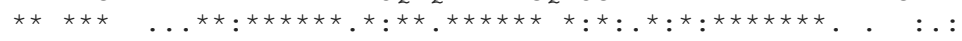

SGEKNVLIFDLGGGTFDVSVLTIDEGSMFEVKATAGDTHLGGEDFDNRLVSHFLQEFKRK 252 KGEKNVLIFDLGGGTFDVPVLTIDEGSMFEVRSTAGDTHLGGEDFDNRLVEHFLQEFQRK 253 DTEQHILIFDLGGGTFDVS ILAIEDG-VFEVLATAGDTHLGGEDFDNRMVNYFTQEFKRK 249 GGEKNILIFDLGGGTFDVSILTIEEG-IFEVKSTAGDTHLGGEDFDNRLVNHFVEEFKRK 248 EGEKNILVFDLGGGTFDVSLLTIDNG-VFEVVATNGDTHLGGEDFDQRVMEHFIKLFKKK 274 EGEKNILVFDLGGGTFDVSLLTIDNG-VFEVVATNGDTHLGGEDFDNRVMEHFIKLFKKK 274 EGEKNILVFDLGGGTFDVSLLTIDNG-VFEVVATNGDTHLGGEDFDNRVMEHFIKLFKKK 245 AGDKIIAVYDLGGGTFDVSILEIQKG-VFEVKSTNGDTFLGGEDFDHALVHHLVGEFKKE 272 -DDKI IAVYDLGGGTFDISILEIQKG-VFEVKSTNGDTFLGGEDFDNALVNFLVNEFKRD 224 -EDKVIAVYDLGGGTFDISILEIQKG-VFEVKSTNGDTFLGGEDFDQALLRHIVKEFKRE 293 -EDKVIAVYDLGGGTFDISILEIQKG-VFEVKSTNGDTFLGGEDFDQALLRHIVKEFKRE 293

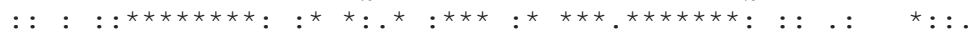

HNKDISKNARATRRLRTACERAKRTLSSSSEASIEIDSLFE----GMDYYTKISRARFEE 308 TRKDISNNTRAMRRLHTACERAKRTLSSSTEASIEIDSLYE----GVDFYSKISRARFEE 309 FGKDLSKSSRALRRLRTACERAKRTLSSSAEANIEIDALFE----GFDFYSKITRARFED 305 NKKDITSNKRALRRLRTACERAKRTLSSSAQANIEIDSLHE----GVDFYTSITRARFEE 304 TGKDVRKDHRAVQKLRREVEKAKRTLSSQHQTKIEIESFFD----NEDFSETLTRAKFEE 330 TGKDIRKDNRAVQKLRREVEKAKRTLSTQFDTRIEIESFFD----GEDFSETFTRARFEE 330 
bioRxiv preprint doi: https://doi.org/10.1101/2020.09.21.307264; this version posted April 22, 2021. The copyright holder for this preprint (which was not certified by peer review) is the author/funder, who has granted bioRxiv a license to display the preprint in perpetuity. It is made available under aCC-BY-NC-ND 4.0 International license.

BCHSC70-2

Celegans HSP70F Daphnia_-̄HSP70c5-1 Pongo_HSP70m Hsapiens_HSP70m

Aplysia HSP70-2 Haliotis_HSP70 Lottia HS̄P70-4 Bplicatilis_HSP70-1 Avaga_HSP70erA Bibericus_HSC70 BCHSC70-2 Celegans HSP70F

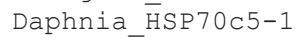
Pongo HSP70m Hsapiēns_HSP70m

Aplysia_HSP70-2 Haliotis HSP7O Lottia HS̄P70-4 Bplicatilis_HSP70-1 Avaga_HSP70ēerA Bibericus HSC70 BCHSC70-2 Celegans HSP70F Daphnia_Ē $\mathrm{H}$ SP70c5-1 Pongo HS $\mathrm{H}$ 70m Hsapiens_HSP70m

Aplysia_HSP70-2 Haliotis HSP70 Lottia_HS̄P70-4 Bplicatilis HSP70-1 Avaga_HSP70ēerA Bibericus HSC70 BCHSC70-2 Celegans HSP70F Daphnia_H̄SP70c5-1 Pongo HS $\bar{S} 70 \mathrm{~m}$ Hsapiens_HSP70m

Aplysia_HSP70-2 Haliotis HSP70 Lottia_HS̄P70-4 Bplicatilis HSP70-1 Avaga_HSP70ēerA Bibericus HSC70 BCHSC70-2 Celegans HSP70F Daphnia_HSP70c5-1 Pongo_HSP $70 \mathrm{~m}$ Hsapiens_HSP70m

Aplysia_HSP70-2 Haliotis HSP70 Lottia HSPP70-4 Bplicatilis_HSP70-1 Avaga_HSP70erA Bibericus HSC70 BCHSC 70-2 Celegans HSP70F Daphnia HSP70c5-1 Pongo_HS̄P70m Hsapiens HSP70m
TGKDIRKDNRAVQKLRREVEKAKRTLSTQFDTRIEIESFFD----SEDFSETFTRASFEK 301 QGVDLTKDPQAMQRLREAAEKAKCELSSTTQTDINLPYITMDQSGPKHLNLKLTRAKFEQ 332 QGLDITKDPMAMQRVKEAAEKAKIELSSSMQTDINLPYLTMDSSGPKHMNLKMSRSKLES 284 TGVDLTKDNMALQRVREAAEKAKCELSSSVQTDINLPYLTMDSSGPKHLNMKLSRAQFEG 353 TGVDLTKDNMALQRVREAAEKAKCELSSSVQTDINLPYLTMDSSGPKHLNMKLTRAQFEG 353

$$
{ }^{\star}: \ldots \quad{ }^{*}:::: \quad{ }^{*}:{ }^{*} \quad{ }^{*}: \quad::{ }^{*}:: \quad: \quad . \quad:^{*}::^{*}
$$

LCADLFRSTLEPVESALRDAKLDKGKIDEVVLVGGSTRIPKIQKLLSDFFNGKELNKSIN 368 LCSDLFRSTLEPVEKALRDAKLDKSCI HDVVLVGGSTRI PKIQKLLQNFMNGKDLNKS IN 369 LCADLFRNTMVPVEKALKDAKLDKSKIDEVVLVGGSTRI PKIQKLLKDFMNGKELNKS IN 365 LCADLFRGTLDPVEKALRDAKMDKSSVNEIVLVGGSTRIPKVQKLLQDFFNGKELNKS IN 364 LNMDLFRSTMKPVQKVLEDADMKKSDIAEVVLVGGSTRIPKVQQLVKDFFDGKEPSRGIN 390 LNMDLFRNTLKPVQKVLEDAGLKKTEIDEIVLVGGSTRI PKIQQLVKEYFDGKEPSRGIN 390 LNIELFRNTLKPVQKVLEDAGLKKTDIDEIVLVGGSTRIPKVQELVKEFFNGKEPSRGIN 361 IVGDLIKRTIEPCRKALHDAEVKSSQIADVLLVGGMSRMPKVQATVQEIF-GKVPSKAVN 391 LVGDLIKRTVGPCQKALKDAEVSKNDIGDVLLVGGMTRMPKVQETVKDIF-GRVPSKAVN 343 IVTDLIRRTIAPCQKAMQDAEVSKSDIGEVILVGGMTRMPKVQQTVQDLF-GRAPSKAVN 412 IVTDLIRRTIAPCQKAMQDAEVSKSDIGEVILVGGMTRMPKVQQTVQDLF-GRAPSKAVN 412

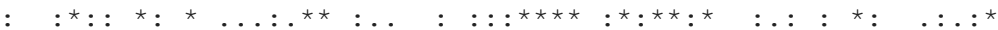

PDEAVAYGAAVQAAVLTGDKSEAIRDVLLVDVAPLSLGIETAGGVMTSLIKRGSTIPAKT 428 PDEAVAYGAAVQAAVLSGDSSDAIKDVLLVDVAPLSLGIETAGGVMTKLIDRNTRIPTKA 429 PDEAVAYGAAVQAAVLIGDKSEKIKDVLLVDVAPLSLGIETAGGVMTRIIERNTRIPTKH 425 PDEAVAYGAAVQAAILTGDKSEAVQDLLLLDVAPLSLGIETAGGVMTALIKRNTTIPTKQ 424 PDEAVAYGAAVQAGVLSGEEN--TGDIVLLDVNPLTMGIETVGGVMTKIIPRNTVIPTKK 448 PDEAVAYGAAVQGGVLSGEDV--GGEVVLLDVNPLTMGIETVGGVMTKLIPRNTVIPTKN 448 PDEAVAYGAAVQGGVLSGEDV--GGDVVLLDVNPLTMGIETVGGMMTKLISRNTVIPTKK 419 PDEAVAMGAAIQGAVLAGD----VTDVLLLDVTPLSLGIETLGGIMTKLITRNTTIPTKK 447 PDEAVAVGAAIQGGVLSGG----VTDILLLDVTPLSLGIETLGGVFTKLIQRNTTIPTKK 399 PDEAVAIGAAIQGGVLAGD----VTDVLLLDVTPLSLGIETLGGVFTKLINRNTTIPTKK 468 PDEAVAIGAAIQGGVLAGD----VTDVLLLDVTPLSLGIETLGGVFTKLINRNTTIPTKK 468

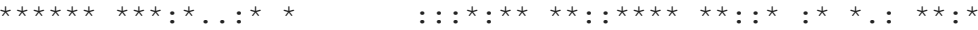

SQVFTTYSDNQPGVDIQVFEGERAMTKDNNLLGKFQLSGIPPAPRGVPQIEVTFDVDANG 488 SQIFTTYSDNQPGVSIQVFEGERALTKDNNILGKFELTGIPPAPRGVPQIEVTFNIDANG 489 SQIFTTYSDNQPGVS IQVYEGERTMTKDNHPMGNFELSGIPPAPRGVPQIEVSFDIDANG 485 TQTFTTYADNQPGVLIQVYEGERAMTKDNHLLGKFELSGIPPAPRGVPQIEVTFDIDANG 484 SQVFSTAAENQPSVTIQVFEGERPMTKDNHVLGKFDLTGIPPAPRGVPQIEVTFEIDVNG 508 -PKFSTASDNOPTVTIAVYEGERPMTKDNHLLGKFDLTGIPPAPRGVPOIEVTFEIDANG 507 SQVFSTAADNQPSVTIAVYEGERPMTKDNHLLGKFDLTGIPPAPRGVPQIEVTFEIDVNG 479 SQVFSTAADGQTQVQIKVFQGEREMATSNKLLGQFSLVGIPPAPRGVPQVEVTFDIDANG 507 SQVFSTAADGQTQVEIKVYQGEREMAADNKILGQFSLIGIPPAPRGVPQIEVTFDIDANG 459 SQVFSTAADGQTQVEIKVCQGEREMAGDNKLLGQFTLIGIPPAPRGVPQIEVTFDIDANG 528 SQVFSTAADGQTQVEIKVCQGEREMAGDNKLLGQFTLIGIPPAPRGVPQIEVTFDIDANG 528

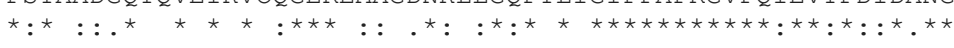

ILNVTAQDKSTGKSSHITIKNERGRLSQAEIDRMLADADKYKDEDEKQRERVSARNQLET 548 ILNVSAVDKSTGKSNNVTISDDQSRLSKADIDRMVSEAERYKEEDEKHKERIAARNHLEN 549 ILNVLAEDKSTGKSNKITITNDKGRLSSEEIEKLVQEAEKYKDEDEKQKRKISARNQLES 545 ILNVSAADKSTGKTNKITITNDKGRLSKEEIDRMVNEAEKYKKDDEEQRDKVAAKNSLES 544 ILKVTAEDKGTGNKNNIVINSNTNRLSPDEIDRMIKDSEKFADEDKKVKERVDAKNELES 568 ILKVSAEDKGTGNKNNIVINNNQNRLSPEEIERMIKDAEKFADEDKKVKEKVEAKNELES 567 ILKVTAEDKGTGNKNNIVINNNQNRLSPEEIEKMIKDAEKFADEDKKVKEKVEAKNELES 539 IVNVSARDRGTGKEQQIVIQS-SGGLSKDQIENMIKEAEKNAAEDAKRKELVEVINQAEG 566 IVHVSARDKGTGKEQQIVIQS-SGGLSKDEIENMVRNAEQFAKEDQVKRDRVEAVNHAEG 518 IVHVSAKDKGTGREQQIVIQS-SGGLSKDDIENMVKNAEKYAEEDRRKKERVEAVNMAEG 587 IVHVSAKDKGTGREQQIVIQS-SGGLSKDDIENMVKNAEKYAEEDRRKKERVEAVNMAEG 587

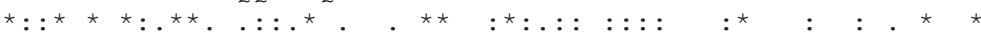

YIFHVKQAV-DA--AGDKLPSPDKDAVLKACNDSLSWLDNNS LADKEEVEDRLKELQKTT 605 YVFSVRASV-DE--MGSKQEDVDKQTVSKACEETLRWLDNNSLAEKEEFEQQYKQLEKLC 606 YVFNVKQVL-DNG-GADKICDSDKETVKQCCDETIQWLDNNALAEKDEYEHRLDEVQKVC 603 YCFNMKQTV-EDEKLAAKISADDKKKILDACEEALKWLDSNQTAEKDEFEHKMKEVEKIC 603 YAYSLKTQLNDKEKLGGKLSSDDKETIEKAVEEQIKWLESNQGAEVDELKEHKKQLEEIV 628 YVYSLKNQLNDKEKLGNKLSEDEKETINSAVDEKIKWLESNSDAEVDDFKEQKKALEEIV 627 YVYSLKNQLSDKEKLGGKLSEEDKEKIQSSVEEKIKWLESNSNAEVEEFKDQKKELEEIV 599 I IHDTEAKMT---EFADQLPKDECEALRTKIADTKKILDNKDNETPEAIKEACNTLQQQS 623 IVHDTESKME---EFKEQLPAEECSKLREQISAVRDMLVNKDSKTPEEIKKTTNDLQQAS 575 I IHDTETKME---EFKDQLPADECNKLKEEISKMRELLARKDSETGENIRQAASSLQQAS 644 I IHDTETKME---EFKDQLPADECNKLKEEISKMRELLARKDSETGENIRQAASSLQQAS 644

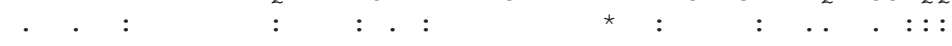


bioRxiv preprint doi: https://doi.org/10.1101/2020.09.21.307264; this version posted April 22, 2021. The copyright holder for this preprint (which was not certified by peer review) is the author/funder, who has granted bioRxiv a license to display the preprint in perpetuity. It is made
available under aCC-BY-NC-ND 4.0 International license.

Drosophila Bb AF295957

Drosophila_Aa_AF295933 Spodoptera FJ $\overline{7} 54276$

Bombyx_AB035326

Paralvinella EF580992

B_plicatilis_HSP70-1_AB775784

B plicatilis HSP70-2 AB775785

A brightwellì HSP70 KXX119429

Wüchereria_AF 167352

Setaria AF079360

C_elegañs_HSP70A_M18540

$\mathrm{C}^{-}$elegans ${ }^{-} \mathrm{HSP}-1$ NM 070667

M_nipponense_KC $\overline{4} 60 \overline{3} 43$

M nipponense DQ660140

Mȳtilus_HSP7 $\overline{0}$ AY861684

Perinerēis KŪ255783

Paralvinelía HSP70-2 EF580993

Haliotis FJ8 $\overline{1} 2176$

Oncorhynchus HSC71 AAB21658

$\mathrm{X}$ maculatus $\overline{\mathrm{H} S C 70} \overline{\mathrm{A} B} 062115$

Human HSPA8 HSC71 NP 006588

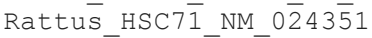

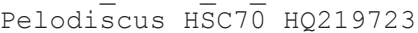

BOS_HSP70_Ū09861

Humān_HSPĀ1A_NM_005345

Rattus_HSP70_1A_NM_031971

$\mathrm{X}$ maculatus $\overline{\mathrm{H} S P} \overline{\mathrm{T}} \mathrm{O}-\overline{1}$ AB0 62113

$X$ maculatus HSP70-2 AB0 62114

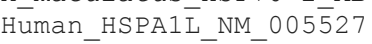

Human HSPA 6 NP 002146

Alligātor_HS̄P7Ō_AB306279

Drosophila_Bb_AF295957

Drosophila_Aa_AF295933

Spodoptera_FJ754276

Bombyx_AB03 5326

Paralvinella_EF580992

B_plicatilis_HSP70-1_AB775784

B_plicatilis_HSP70-2_AB775785

A brightwelli $\bar{i}$ HSP70 $\bar{k} \times 119429$

Wüchereria_AF'167352

Setaria AF $\overline{0} 79360$

C_elegans_HSP70A_M18540

C elegans HSP-1 NM 070667

M_nipponense_KC $\overline{4} 60 \overline{3} 43$

Mnipponense DQ660140

Mytilus_HSP70_AY861684

Perinerēis KU 255783

Paralvinelīa_HSP70-2_EF580993

Haliotis_FJ812176

Oncorhynchus_HSC71 AAB21658

$\mathrm{X}$ maculatus $\overline{\mathrm{H} S C 70} \overline{\mathrm{AB}} 062115$

Human HSPA8 ${ }_{\text {HSC71 NP } 006588}$

Rattus_HSC7 $\overline{1}$ NM $0 \overline{2} 43 \overline{5} \overline{1}$

Pelodiscus HS $\mathrm{C} 7 \overline{0}$ HQ219723

Bos_HSP70_Ū09861

Humān_HSPĀ1A_NM_005345

Rattus HSP70_1A_NM 031971

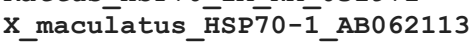

$X$ maculatus_HSP70-2_AB062114

Human_HSPA1 $\overline{\mathrm{L}}$ NM $005 \overline{5} 27$

Human HSPA $6 \overline{\mathrm{N} P} \overline{0} 02146$

Alligator_HS̄P70_AB306279

Drosophila Bb AF295957

Drosophila_Aa_AF295933

Spodoptera_FJ754276

Bombyx_AB03 35326

Paralvinella_EF580992

B_plicatilis_HSP70-1_AB775784

B_plicatilis_HSP70-2_AB775785

A brightwelli $\bar{i}$ HSP70 $\bar{K} \times 119429$

Wüchereria_AF'167352

Setaria AF $\mathbf{0} 79360$

C_elegañs_HSP70A_M18540

C_elegans_HSP-1_ $\mathbf{N} M$ _070667

M_nipponense_KC $\overline{4} 60 \overline{3} 43$
-_-_-n-_-nATGCCTGCTATTGGAATCGATCTGGGCACCACCTACTCCTGCGTG 45 ----n---n----ATGCCTGCTATTGGAATCGATCTGGGCACCACCTACTCCTGCGTG 45 --n-n--n---ATGCCAGCCATTGGAATAGATCTGGGTACCACATACTCGTGCGTC 45 -ATGCCAGCTATCGGAATCGATTTGGGAACGACATACTCATGTGTT 45 ATGGCACAAGGAAAAACACCGGCAATTGGCATTGATCTTGGCACCACCTATTCCTGTGTT 60 -----ATGAGCAAAGGACCCGCTGTAGGCATCGACTTGGGCACCACGTACTCTTGCGTG 54 -----ATGAGCAAAGGACCCGCTGTAGGCATCGACTTGGGCACCACGTACTCTTGCGTG 54 -------ATGGCCAAAACAGCGATCGGTATTGATTTGGGCACGACATATTCGTGTGTG 51 -------ATGTCAAAGAATGCCATTGGTATCGATCTTGGAACCACGTATTCCTGCGTG 51 -------ATGTCAAAGAACGCAATCGGCATTGATCTTGGGACTACTTACTCGTGCGTG 51 -----ATGAGTAAGCATAACGCTGTTGGAATCGATTTGGGAACTACCTACTCCTGCGTG 54 -----ATGAGTAAGCATAACGCTGTTGGAATCGATTTGGGAACTACCTACTCCTGCGTG 54 -----ATGGCTAAAAGTGCAGCTGTCGGGATTGACCTGGGTACCACCTACTCATGTGTA 54 -----ATGGCTAAAAGTGCAGCTGTCGGGATTGACCTGGGTACCACCTACTCATGTGTA 54 ---ATGGCAAAAACAGGACCAGCAATTGGAATAGATCTTGGAACTACATACTCCTGTGTT 57 -...-.--ATGGCAGCACCTGCAGTAGGTATTGATCTCGGCACCACATACTCCTGCGTT 51 -----ATGGCGAAGGCAAGTGCTGTGGGTATCGACCTTGGAACCACGTACTCTTGCGTT 54 -----ATGGCCAAGGCACCAGCTATTGGTATAGATCTGGGGACCACCTACTCGTGTGTA 54 --_--ATGTCTAAGGGACCAGCAGTCGGCATCGATCTCGGGACCACCTACTCCTGCGTG 54 -----ATGTCCAAGGGACCAGCTGTAGGCATTGACCTGGGCACTACCTACTCTTGTGTG 54 -----ATGTCCAAGGGACCTGCAGTTGGTATTGATCTTGGCACCACCTACTCTTGTGTG 54 -----ATGTCTAAGGGACCTGCAGTTGGCATTGATCTTGGCACCACCTACTCCTGTGTG 54 -----ATGTCTAAGGGACCAGCAGTTGGAATTGATCTTGGCACCACATACTCCTGTGTT 54 -----ATGGCGAAAAACATGGCTATCGGCATCGACCTGGGCACCACCTACTCCTGCGTA 54 -----ATGGCCAAAGCCGCGGCGATCGGCATCGACCTGGGCACCACCTACTCCTGCGTG 54 -----ATGGCCAAGAAAACAGCGATCGGCATCGACCTGGGCACCACCTACTCGTGCGTG 54 ATGTCATCAGCTAAGGGAATATCCATTGGCATCGATCTGGGCACCACCTACTCTTGTGTC 60 ATGTGTGCAGCTAAAAACGTGGCCATCGGCATCGACCTGGGCACCACCTACTCCTGCGTG 60 ATGGCTACTGCCAAGGGAATCGCCATAGGCATCGACCTGGGCACCACCTACTCCTGTGTG 60 ATGCAGGCCCCACGGGAGCTCGCGGTGGGCATCGACCTGGGCACCACCTACTCGTGCGTG 60 ---ATGTCGGGCAAAGGGCCTGCGATCGGCATCGACTTGGGCACCACGTACTCCTGCGTG 57

GGTGTCTACCAGCATGGCAAGGTTGAGATTATCGCCAATGACCAGGGCAACCGCACCACG 105 GGTGTCTACCAGCATGGCAAGGTGGAGATTATCGCCAACGACCAGGGCAACCGCACCACG 105 GGCGTGTGGCAGCACGGCAACGTGGAGATCATCGCCAACGACCAGGGCAACCGCACCACA 105 GGAGTATGGCAGCACGGGAACGTGGAGATCATCGCGAACGACCAGGGCAACCGTACCACA 105 GGTGTCTTCCAACATGGAAAAGTGGAGATTATTGCCAACGACCAAGGAAATAGGACGACA 120 GGCGTATTCCAGCACGGCAAAGTGGAAATAATCGCCAACGACCAAGGCAACCGCACAACC 114 GGCGTATTCCAGCACGGCAAAGTGGAAATAATCGCCAACGACCAAGGCAACCGCACAACC 114 GGCGTGTTCCAACATGGTAAAGTTGAGATCATTGCCAACGACCAAGGTAATAGAACAACG 111 GGTGTGTTCATGCACGGCAAAGTGGAAATCATTGCTAATGATCAAGGTAATCGTACAACA 111 GGTGTTTTCATGCACGGTAAAGTGGAAATCATTGCTAACGATCAAGGTAATCGAACAACA 111 GGAGTTTTCATGCACGGAAAGGTAGAAATCATTGCCAACGATCAAGGAAACCGTACAACT 114 GGAGTTTTCATGCACGGAAAGGTAGAAATCATTGCCAACGATCAAGGAAACCGTACAACT 114 GGTGTGTTCCAGCATGGAAAGGTAGAAATCATTGCCAATGATCAGGGCAACCGAACCACT 114 GGTGTGTTCCAGCATGGAAAGGTAGAAATCATTGCCAATGATCAGGGCAACCGAACCACT 114 GGAGTATTCCAGCATGGCAAAGTTGAAATCATTGCCAATGATCAAGGAAACAGAACAACC 117 GGGGTTTTCCAGCACGGGAAAGTGGAAATCATTGCAAACGACCAGGGTAACAGGACCACG 111 GGTGTATTCCAGCATGGCAAGGTGGAAATCGTAGCTAATGATCAGGGAAACCGAACGACG 114 GGGGTATTTCAACACGGAAAGGTAGAAATCATTGCCAATGACCAAGGAAACAGAACAACA 114 GGTGTGTTCCAGCATGGCAAGGTTGAAATCATTGCCAACGACCAAGGCAACAGGACCACT 114 GGAGTGTTTCAGCACGGGAAAGTTGAGATCATTGCTAATGACCAGGGGAACAGGACCACG 114 GGTGTTTTCCAGCACGGAAAAGTCGAGATAATTGCCAATGATCAGGGAAACCGAACCACT 114 GGTGTCTTCCAGCATGGAAAGGTGGAAATAATTGCCAATGACCAGGGTAACCGCACCACG 114 GGGGTCTTCCAACATGGCAAGGTGGAGATCATTGCCAATGATCAGGGCAACAGGACCACG 114 GGGGTGTTCCAGCACGGCAAGGTGGAGATCATCGCCAACGACCAGGGCAACCGCACCACC 114 GGGGTGTTCCAACACGGCAAGGTGGAGATCATCGCCAACGACCAGGGCAACCGCACCACC 114 GGCGTGTTCCAGCACGGCAAGGTGGAGATCATCGCCAACGACCAGGGCAACCGCACGACC 114 GGGGTCTTCCAGCATGGAAAAGTGGAAATTATCGCCAATGATCAAGGCAACAGGACAACT 120 GGGGTTTTCCAGCACGGAAAAGTGGAAATCATCGCCAACGACCAGGGCAACAGGACCACC 120 GGGGTGTTCCAGCACGGCAAGGTGGAGATCATCGCCAACGACCAGGGCAACCGCACCACC 120 GGCGTGTTTCAGCAGGGCCGCGTGGAGATCCTGGCCAACGACCAGGGCAACCGCACCACG 120 GGCGTCTTCCAGCATGGCAAGGTGGAGATCATTGCCAACGACCAGGGCAACCGCACCACG 117

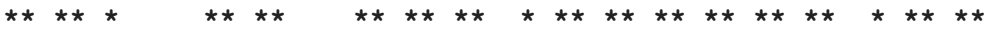

CCGTCCTACGTGGCTTTCACAGACTCGGAACGCCTCATTGGTGATCCGGCCAAGAACCAG 165 CCGTCCTACGTGGCTTTCACAGATTCGGAACGCCTCATCGGCGATCCGGCTAAGAACCAG 165 CCATCCTATGTGGCGTTCACGGACACGGAGCGCCTCATCGGAGACGCAGCCAAGAACCAG 165 CCATCGTACGTCGCGTTCACGGACACGGAGCGTCTCATCGGCGACGCAGCCAAGAACCAG 165 CCAAGTTATGTGGCATTTACAGATACCGAGAGACTGATAGGTGATGCGGCAAAGAACCAA 180 СССTCCTACGTAGCGTTCACCGACACCGAGCGCTTGATCGGCGACGCGGCCAAAAACCAA 174 CCСTCCTACGTAGCGTTCACCGACACCGAGCGCTTGATCGGCGACGCGGCCAAAAACCAA 174 CCGAGTTACGTGGCGTTCACTGATACTGAACGTTTGATCGGAGACGCGGCCAAGAACCAA 171 CCATCGTATGTGGCATTTACCGATACTGAACGTCTTATTGGCGATGCAGCCAAAAATCAG 171 CCATCATATGTGGCATTTACCGATACTGAACGTCTTATTGGTGATGCAGCCAAAAATCAG 171 CCATCATATGTGGCTTTCACCGACACCGAGCGTCTCATCGGAGATGCTGCCAAGAATCAA 174 CCATCATATGTGGCTTTCACCGACACCGAGCGTCTCATCGGAGATGCTGCCAAGAATCAA 174 CCATCATATGTTGCTTTCACCGATACAGAGCGTCTTATTGGTGATGCTGCCAAGAACCAA 174 
bioRxiv preprint doi: https://doi.org/10.1101/2020.09.21.307264; this version posted April 22, 2021. The copyright holder for this preprint (which was not certified by peer review) is the author/funder, who has granted bioRxiv a license to display the preprint in perpetuity. It is made available under aCC-BY-NC-ND 4.0 International license.

M nipponense D0660140

Mytilus_HSP70_AY861684

Perinerēis KU $\overline{2} 55783$

Paralvinelīa_HSP70-2_EF580993

Haliotis FJ8 $\mathbf{1} 2176$

Oncorhynchus_HSC71_AAB21658

$\mathrm{X}$ maculatus $\overline{\mathrm{H}} \mathrm{SC70}$ A $\mathrm{A} 062115$

Hüman_HSPA8_HSC71_NP_006588

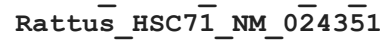

Pelodiscus_HSC70__HQ219723

BOS_HSP70_Ū09861

Human HSPA 1 A NM 005345

Rattus'HSP70_1A_NM_031971

$X$ maculatus $\bar{H} S P \overline{7} 0-\overline{1}$ AB0 62113

$X$ maculatus_HSP70-2 AB062114

Hüman HSPA1 $\overline{\mathrm{I}}$ NM $005 \overline{5} 27$

Human_HSPA6_N $\overline{\mathrm{N}}$-002146

Alligātor_HS̄P7Ō_AB306279

Drosophila_Bb_AF295957

Drosophila_Aa_AF295933

Spodoptera FJ $\overline{7} 54276$

Bombyx_AB $0 \overline{3} 5326$

Paralvinella EF580992

B_plicatilis_HSP70-1_AB775784

B plicatilis HSP70-2 AB775785

A_brightwellìi_HSP70_KX119429

Wüchereria_AF'167352

Setaria_AF $\overline{0} 79360$

C_elegañs_HSP70A_M18540

C_elegans_HSP-1 NM 070667

M_nipponense_KC $\overline{4} 60 \overline{3} 43$

M nipponense DQ660140

Mýtilus_HSP70̄_AY861684

Perinerēis KU 255783

Paralvinelīa_HSP70-2_EF580993

Haliotis_FJ81̄2176

Oncorhynchus_HSC71_AAB21658

$\mathrm{X}$ maculatus $\overline{\mathrm{H} S C 70} \overline{\mathrm{A}} \mathrm{B} 062115$

Human_HSPA8_HSC71_NP_006588

Rattus_HSC7 $\overline{1}$ NM_O $0 \overline{2} 43 \overline{5} 1$

Pelodiscus HS C70 HQ219723

Bos_HSP70_Ū09861

Humān HSPĀ 1 A NM 005345

Rattus_HSP70_1A_NM_031971

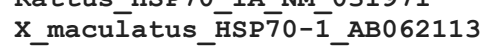

X_maculatus_HSP70-2_AB062114

Human HSPA1 $\overline{\bar{L}}$ NM $005 \overline{5} 27$

Human_HSPA6_N $P$-002146

Alligātor_HS̄P70_AB306279

Drosophila Bb AF295957

Drosophila_Aa_AF295933

Spodoptera FJ754276

Bombyx_AB0 $\overline{3} 5326$

Paralvinella_EF580992

B_plicatilis_HSP70-1_AB775784

B_plicatilis_HSP70-2_AB775785

A_brightwellìi_HSP70_K $\mathrm{K} \times 119429$

Wüchereria_AF'167352

Setaria_AF-79360

C_elegañs_HSP70A_M1 8540

C elegans HSP-1 NM_070667

M_nipponense_KC $\overline{4} 60 \overline{3} 43$

M-nipponense DQ660140

Mȳtilus_HSP70_AY861684

Perinerēis_KU 255783

Paralvinelīa_HSP70-2_EF580993

Haliotis_FJ $8 \overline{1} 2176$

Oncorhynchus_HSC71_AAB21658

X_maculatus_ $\mathrm{H} S \mathrm{SC70} \overline{\mathrm{A}} \mathrm{B} 062115$

Hüman HSPA $8^{-}{ }^{-}{ }^{-}{ }^{-}$NP 006588

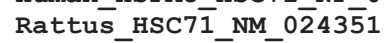

Pelodiscus HS C70 HQ219723

BOS_HSP70_Ū09861

Humān_HSPĀ 1 A_NM_005345

Rattus_HSP70_1A_NM_031971
CCATCATATGTTGCTTTCACCGACACAGAGCGTCTTATTGGTGATGCTGCCAAGAACCAA 174 CCAAGCTATGTCGCCTTCACAGACACCGAAAGATTAATAGGTGATGCTGCCAAGAACCAA 177 CCCAGTTATGTGGCATTCACAGACTCCGAGCGTCTCATTGGAGATGCCGCAAAGAACCAA 171 CCAAGTTATGTGGCGTTTACGGATTCCGAACGTATTATTGGAGATGGAGCCAAGAACCAA 174 CCCAGCTATGTTGCCTTCACCGACACAGAGCGTCTCATTGGTGACGCTGCAAAGAACCAG 174 CCAAGCTACGTTGCCTTCACTGACTCTGAGAGGCTCATCGGTGATGCTGCCAAGAATCAG 174 CCCAGTTATGTGGCCTTTACTGACACAGAGAGGCTGATTGGAGATGCAGCCAAGAACCAG 174 CCAAGCTATGTCGCCTTTACGGACACTGAACGGTTGATCGGTGATGCCGCAAAGAATCAA 174 CCGAGCTATGTTGCTTTCACCGACACAGAACGATTAATTGGGGATGCGGCCAAGAATCAG 174 CCAAGTTACGTTGCCTTCACAGATACAGAGAGGTTGATCGGTGATGCTGCAAAGAACCAA 174 CCCAGCTACGTGGCCTTCACCGATACCGAGCGGCTCATCGGCGATGCGGCCAAGAACCAG 174 CCCAGCTACGTGGCCTTCACGGACACCGAGCGGCTCATCGGGGATGCGGCCAAGAACCAG 174 CCCAGCTACGTGGCCTTCACCGACACCGAGCGGCTCATCGGGGACGCCGCCAAGAACCAG 174 CCCAGCTACGTGGCCTTTACCGACACAGAGAGGCTCATCGGAGATGCTGCCAAGAACCAA 180 CCCAGCTACGTGGCCTTCACCGACACCGAGAGGCTGATCGGGGACGCGGCCAAGAACCAG 180 CCCAGCTACGTGGCCTTCACAGACACCGAGCGGCTCATTGGGGATGCGGCCAAGAACCAG 180 CCCAGCTACGTGGCCTTCACCGACACCGAGCGGCTGGTCGGGGACGCGGCCAAGAGCCAG 180 CCCAGCTACGTGGCCTTCACGGACACGGAGCGCCTCATCGGCGACGCGGCCAAGAACCAG 177

GTGGCCATGAACCCCAGAAACACAGTGTTTGACGCCAAGCGACTCATCGGCCGAAAATAC 225 GTGGCCATGAACCCCAGAAACACAGTGTTTGACGCCAAGCGACTCATCGGCCGAAAATAC 225 GTCGCCCTCAACCCCAACAACACTGTGTTCGACGCCAAGCGACTGATCGGAAGGAAATTC 225 GTCGCCTTGAACCCTAACAACACCGTGTTCGACGCGAAGGGGCTGATCGGGAGGAAATTC 225 GTGGCGCTGAATCCGAGCAACACGGTATTTGATGCGAAGAGGCTGATTGGTCGAAGATTT 240 GTGGCCATGAACCCCAATAACACGGTGTTCGACGCCAAGCGTCTCATCGGCCGCAAGTTC 234 GTGGCCATGAACCCCAATAACACGGTGTTCGACGCCAAGCGTCTCATCGGCCGCAAGTTC 234 GTGGCCATGAATCCGACGAACACAGTGTTCGACGCGAAACGTCTCATCGGACGCAGATTC 231 GTTGCAATGAATCCGCACAACACTGTCTTCGACGCTAAACGACTGATTGGTCGTAAGTTT 231 GTTGCGATGAATCCGCACAATACTGTTTTCGATGCGAAACGATTGATCGGCCGTAAATTT 231 GTTGCCATGAACCCACATAACACTGTTTTCGATGCCAAACGTCTTATTGGACGCAAGTTC 234 GTTGCCATGAACCCACATAACACTGTTTTCGATGCCAAACGTCTTATTGGACGCAAGTTC 234 GTTGCTATGAATCCCAACAACACTGTATTTGATGCCAAGAGGCTCATTGGTCGTAAGTTT 234 GTTGCTATGAATCCCAACAACACTGTATTTGATGCCAAGAGGCTCATTGGTCGTAAGTTT 234 GTGGCAATGAACCCAGTCAACACGGTTTTTGATGCCAAGAGATTAATTGGTAGAAAGTTT 237 GTGGCCATGAACCCCGAGAACACAGTCTTCGACGCCAAACGTTTGATCGGACGCAAGTTT 231 GTGGCCATGAACCCCAGTAACACGGTGTTCGATGCGAAACGTCTCATTGGTCGTAGGCTC 234 GTTGCCATGAACCCAGAGAACACTATCTTCGATGCCAAACGTCTGATTGGTAGAAGATTT 234 GTTGCCATGAACCCCTGCAACACAGTATTCGATGCTAAGAGACTGATTGGCCGCAGGTTT 234 GTGGCCCTCAACCCCAACAACACCGTTTTTGATGCAAAGCGGCTTATTGGGCGTCGTTTT 234 GTTGCAATGAACCCCACCAACACAGTTTTTGATGCCAAACGTCTGATTGGACGCAGATTT 234 GTTGCAATGAACCCCACCAACACAGTTTTTGATGCCAAACGTCTGATCGGACGTAGGTTC 234 GTTGCAATGAATCCTACCAACACGGTTTTTGATGCAAAGCGGCTGATCGGTCGTAGATTT 234 GTGGCGCTGAACCCGCAGAACACGGTGTTCGACGCGAAGCGGCTGATCGGCCGCAAGTTC 234 GTGGCGCTGAACCCGCAGAACACCGTGTTTGACGCGAAGCGGCTGATTGGCCGCAAGTTC 234 GTGGCGCTGAACCCGCAGAACACCGTGTTCGACGCGAAGCGGCTGATCGGCCGCAAGTTC 234 GTTGCCATGAACCCCACCAACACGATCTTTGATGCCAAACGGCTCATTGGAAGAAGGTTT 240 GTGGCCCTGAACCCCAGCAACACGGTGTTTGACGCCAAGAGGCTGATCGGCAGAAAGTTT 240 GTAGCAATGAATCCCCAGAACACTGTTTTTGATGCTAAACGTCTGATCGGCAGGAAATTT 240 GCGGCCCTGAACCCCCACAACACCGTGTTCGATGCCAAGCGGCTGATCGGGCGCAAGTTC 240 GTGGCCATGAACCCCACCAACACCATCTTCGACGCCAAGCGCCTCATCGGCCGCAAGTAC 237

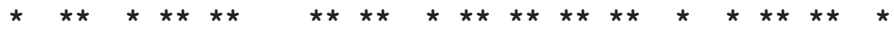

GACGATCCCAAAATCGCAGAGGACATGAAGCACTGGCCTTTCAAAGTTGTAAGCGACG- - 283 GACGACCCCAAGATCGCAGAGGACATGAAGCACTGGCCTTTCAAGGTGGTAAGCGACG- - 283 GATGACCCCAAGATCCAGGCAGACATGAAGCACTGGCCCTTCAGGGTGGTCAGCGACT- - 283 GACGACCCCAAGATTCAGCAGGACATGAAGCACTGGCCCTTCAAAGTAATCAACGACT-- 283 GATGACGACAATGTCCAGAAGGACATCAAACATTGGCCTTTTAAAGTACTTAACGACG-- 298 GACGACCTGACCGTCCAGGCCGACATGAAGCACTGGCCCTTCACGGTGATCAGCGACA- - 292 GACGACCCGACCGTCCAGGCCGACATGAAGCACTGGCCCTTCACGGTGATCAGCGACA-- 292 GACGACTCCACTGTCCAGTCCGACATGAAACACTGGCCGTTCACTGTCGTCAATGAAG- - 289 GATGATGGATCAGTGCAATCGGATATGAAACATTGGCCATTTAAAGTAGTGAATGCTGGT 291 GATGATGGATCAGTACAGTCAGACATGAAACATTGGCCGTTTAAAGTTATGAATGCTGGT 291 GACGATCCAGCAGTTCAGTCTGACATGAAGCATTGGCCATTCAAGGTCATCTCTGCCGAA 294 GACGATCCAGCAGTTCAGTCTGACATGAAGCATTGGCCATTCAAGGTCATCTCTGCCGAA 294 GAAGATCATGTAGTGCAGTCCGACATGAAACATTGGCCCTTTACAGTCATTAATGACA-- 292 GAAGATCATGTAGTGCAGTCCGACATGAAACATTGGCCCTTTACAGTCATTAATGACA-- 292 GATGACGCTACAGTACAATCAGACATGAAGCATTGGCCTTTCACTGTTGTCAATGATG- 295 GACGACTCAGCCGTTCAGTCAGACAAAAAGCATTGGCCATTCGATGTGGTCAGTGAGG-- 289 GATGATTCGTCCGTCCAATCAGATATGAAGTTCTGGCCCTTCAAGGTCATCAGTGAGA-- 292 GATGAGACAAATGTTCAATCAGACATGAAGCACTGGCCATTCAATGTGTTGAGTGATG-- 292 GATGATGGAGTTGTTCAATCGGACATGAAGCATTGGCCCTTTGAAGTTATCAATGATT- 292 GACGATAGTATTGTCCAATCAGACATGAAACATTGGCCCTTCACCATCATCAATGATA-- 292 GATGATGCTGTTGTCCAGTCTGATATGAAACATTGGCCCTTTATGGTGGTGAATGATG- - 292 GATGATGCTGTTGTTCAGTCTGACATGAAGCACTGGCCCTTCATGGTGGTGAACGATG-- 292 GATGATGCTGTTGTCCAGTCGGACATGAAACATTGGCCATTCACTGTGGTGAATGATG- - 292 GGAGACCCGGTGGTGCAGTCGGACATGAAGGAGTGGCCTTTCCGCGTCATCAACGACG- - 292 GGCGACCCGGTGGTGCAGTCGGACATGAAGCACTGGCCTTTCCAGGTGATCAACGACG- - 292 GGCGACCCGGTGGTGCAGTCGGACATGAAGCACTGGCCCTTCCAGGTGGTGAACGACG- - 292 
bioRxiv preprint doi: https://doi.org/10.1101/2020.09.21.307264; this version posted April 22, 2021. The copyright holder for this preprint (which was not certified by peer review) is the author/funder, who has granted bioRxiv a license to display the preprint in perpetuity. It is made available under aCC-BY-NC-ND 4.0 International license.

$X$ maculatus HSP70-1 AB0 62113 X maculatus HSP70-2 AB062114 Human HSPA1 $\overline{\mathrm{L}}$ NM $005 \overline{5} 27$ Human_HSPA6_N $\bar{N}$ _ 002146 Alligätor_HS̄P7O__AB306279

Drosophila_Bb_AF2 95957 Drosophila_Aa-AF295933 Spodoptera FJ 754276 Bombyx_AB0 $\overline{3} 5326$

Paralvinella EF580992 B_plicatilis_HSP70-1_AB775784 B plicatilis HSP70-2 AB775785 A brightwellii_HSP70 $\bar{K} \times 119429$ Wüchereria AF'167352

Setaria_AF079360 C_elegañs_HSP70A M18540 C_elegans_HSP-1_N $\mathrm{NM} 070667$ M_nipponense_KC $\overline{4} 60 \overline{3} 43$ M_nipponense_DQ660140 Mýtilus_HSP70_AY861684 Perinerēis KU 255783

Paralvinelía_HSP70-2_EF580993 Haliotis FJ8 $\overline{1} 2176$

Oncorhynchus_HSC71_AAB21658 $X$ maculatus $\overline{\mathrm{H} S C 70}$ AB062115 Human HSPA8 HSC71 NP 006588 Rattus_HSC7 $\overline{1}$ NM $0 \overline{2} 43 \overline{5} \overline{1}$ Pelodiscus_HS̄C70_HQ219723 BOS_HSP70 Ū09861 Humān HSPĀ 1 A NM 005345 Rattus_HSP70_1A_NM_031971 $X$ maculatus $\bar{H} S P \overline{7} 0-\overline{1}$ AB0 62113 X_maculatus_HSP70-2_AB062114 Human_HSPA1 $\overline{\mathrm{L}}$ NM $005 \overline{5} 27$ Human_HSPA6_NP_002146 Alligātor_HS̄P70_AB306279

Drosophila_Bb_AF295957 Drosophila_Aa_AF295933 Spodoptera FJ754276 Bombyx_AB0 35326 Paralvinella EF580992 B_plicatilis_HSP70-1_AB775784 B plicatilis HSP70-2 AB775785 A_brightwellí_HSP70_KX119429 Wüchereria AF'167352 Setaria_AF079360 C_elegañs_HSP70A_M1 8540 C-elegans HSP-1 $\bar{N}$ M 070667 M_nipponense_KC $\overline{4} 60 \overline{3} 43$ Mnipponense DQ660140 Mȳtilus HSP7 $\overline{0}$ AY861684 Perineréis KU 255783 Paralvinelīa_HSP70-2_EF580993 Haliotis FJ8 $\overline{1} 2176$

Oncorhynchus_HSC71_AAB21658 $X$ maculatus_H $\mathrm{H} C 70$ A $\mathrm{B} 062115$ Human HSPA8 HSC71 NP 006588 Rattus_HSC7 $\overline{1}$ NM $0 \overline{2} 43 \overline{5} \overline{1}$ Pelodiscus HS C7 Bos_HSP70_Ū09861 Humān HSPĀ 1 A NM 005345 Rattus HSP70 1A NM 031971 $X$ maculatus $\bar{H} S P \overline{7} 0-\overline{1}$ AB0 62113 $X$ maculatus HSP70-2 AB062114 Human HSPA1 $\overline{\mathrm{L}}$ NM $005 \overline{5} 27$ Human HSPA $6 \overline{\mathrm{N}} \mathrm{P} \overline{0} 02146$ Alligator_HS̄P7O_AB306279

Drosophila_Bb_AF295957 Drosophila_Aa_AF295933 Spodoptera FJ754276 Bombyx_AB0 35326 Paralvinella EF580992 B_plicatilis_HSP70-1_AB775784
GATGACCTGGTGGTCCAGTCTGACATGAAACTCTGGCCGTTCAAGGTGATCAACGACA- - 298 GAAGAGCCAGTGGTGCAGGCCGACATGAAGCACTGGCCCTTCGAGGTGCTTTCAGACG-- 298 AATGATCCTGTTGTACAAGCAGATATGAAACTTTGGCCTTTTCAAGTGATTAATGAAG- 298 GCGGACACCACGGTGCAGTCGGACATGAAGCACTGGCCCTTCCGGGTGGTGAGCGAGG- 298 GACGACCCCACGGTGCAGGCGGACATGAAGCACTGGCCCTTCCACGTAGTGTCCGAGG- 295$$
\text { ** }
$$

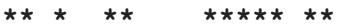$$
\text { * }
$$

-GCGGAAAGCCCAAGATCGGGGTGGAGTATAAGGGTGAGTCCAAGAGATTTGCTCCCGAG 342 -GCGGAAAGCCCAAGATCGGGGTGGAGTATAAGGGTGAGTCCAAGAGATTTGCCCCCGAG 342 -GTGGCAAACCGAAGATCCAAGTGGAGTTCAAGGGTGAAACGAAACGGTTCGCGCCCGAG 342 -GCGGCAAACCGAAAATACAGATCGAGTTCAAAGGTGAGACGAAACGATTTGCGCCAGAA 342 -GTGGAAAGCCAAAGATTGACGTAGAATACAAAAGTGAGAGGAAACGTTTTACTCCAGAA 357 -GCGGCCGGCCCAAAATCCAGGTCGAGTTCAAAGGCGAGACCAAGAGCTTCTACCCGGAA 351 -GCGGCCGGCCCAAAATCCAGGTCGAGTTCAAAGGCGAGACCAAGAGCTTCTACCCGGAA 351 -GCGGAAAACCAAAAATCCAAGTCGAGTTCAAAGGCGAGAAAAAGACCTTCTTCCCTGAA 348 GGTGGTAAACCTAAAGTGCAGGTTGAATACAAGGGTGAGACAAAGACCTTTACTCCAGAA 351 GGAGGCAAGCCAAAAGTTCAAGTGGAGTATAAGGGGGAGACGAAAACTTTCACGCCCGGA 351 GGAGCTAAGCCAAAGGTCCAAGTTGAGTACAAAGGAGAGAACAAGATCTTCACTCCAGAA 354 GGAGCTAAGCCAAAGGTCCAAGTTGAGTACAAAGGAGAGAACAAGATCTTCACTCCAGAA 354 -GCACGAAGCCCAAAATTCAAGTTGATTACAAAGGAGAGACCAAAACCTTTTTCCCAGAA 351 -GCACGAAGCCCAAAATTCAAGTTGATTACAAAGGAGAGACCAAAACCTTTTTCCCAGAA 351 -СCTCCAAACCTAAAGTTACGGTAGACTACAAAGGAGAAACAAAAACATTTTTCCCTGAG 354 -GTGGCAAACCAAAGATCTCTGTTGATTACAAGGGGGAGAAGAAATCATTTTACCCAGAA 348 -ATGGCAAGCCAAAAATCCAAGTGGAGTACAAGGGGGAGTTGAAGACCTTCTATCCCGAA 351 -GAGGCAAACCCAAGATCCAAGTAAATTACAAAGATGAACCAAAAACTTTCTACCCTGAA 351 -СTACTCGGCCTAAGCTCCAAGTTGAATACAAAGGAGAGACTAAGTCCTTCTACCCAGAA 351 -GCTCGAGGCCCAAAGTGAAAGTGGAGTACAAAGGGGAGACGAAGACCTTCTACCCAGAG 351 -CTGGCAGGCCCAAGGTCCAAGTAGAATACAAGGGAGAGACCAAAAGCTTCTATCCAGAG 351 -CAGGCAGGCCCAAGGTCCAAGTCGAATACAAAGGGGAGACAAAAAGTTTCTATCCTGAG 351 -CTGGCAGGCCGAAAGTCCAGGTTGAGTACAAAGGGGAGACCAAGAGCTTCTATCCAGAG 351 -GAGACAAGCCTAAGGTGCAGGTGAGCTACAAAGGGGAGACCAAGGCGTTCTACCCGGAG 351 -GAGACAAGCCCAAGGTGCAGGTGAGCTACAAGGGGGAGACCAAGGCATTCTACCCCGAG 351 -GCGACAAGCCCAAGGTGCAGGTGAACTACAAGGGCGAGAACCGGTCGTTCTACCCGGAG 351 -ACGGAAAGCCCAAAGTCCAGGTGGAATATAAAGGAGAAATTAAGACATTCTGTCCTGAA 357 -GAGGCAGGCCCAAAATTCAGGTGGAGTACAAAGGGGAGAACAAAGCCTTCTTCCCTGAG 357 -GAGGCAAGCCCAAAGTCCTTGTGTCCTACAAAGGGGAGAATAAAGCTTTCTACCCTGAG 357 -GCGGCAAGCCCAAGGTGCGCGTATGCTACCGCGGGGAGGACAAGACGTTCTACCCCGAG 357 -GCGGCAAGCCCAAGGTGCAGGTGGAGTATAAGGGCGAGGCCAAGACCTTCTTCCCCGAA 354

$$
\text { ** } * * * * \text { * } * * \text { * } * * *
$$

GAGATCAGTTCGATGGTGCTGACCAAGATGAAGGAGACGGCGGAGGCGTATCTGGGCGAG 402 GAGATCAGCTCGATGGTGCTGACCAAGATGAAGGAGACGGCGGAGGCATATCTGGGCGA 402 GAGATCAGCAGCATGGTGCTGACGAAGATGAAGGAGACGGCGGAAGCTTACCTCGGAACG 402 GAAATTAGCAGCATGGTGCTGACAAAAATGAAGGAGACGGCGGAAGCCTATCTGGGAAGT 402 GAAATCAGCTCGATGATACTTACCAAGATGAAAGAGACGGCCGAGGCCTATCTCGGTAAT 417 GAAGTCTCATCCATGGTACTGACCAAAATGAAGGAGACGGCCGAGGCCTACCTGGGCAAA 411 GAAGTCTCATCCATGGTACTGACCAAAATGAAGGAGACGGCCGAGGCCTACCTGGGCAAA 411 GAAATCTCATCAATGGTCTTAATTAAAATGAAAGAGATCGCCGAGGCTTATTTGGGAAAG 408 GAGATTTCGTCGATGGTTTTGGTTAAAATGAAGGAGACGGCTGAAGCTTTTCTGGGTCAT 411 GAAATTTCTTCAATGGTTTTAGTTAAAATGAAAGAAACAGCTGAGGCATTTCTTGGTCAC 411 GAGATCTCCTCAATGGTTCTGCTGAAGATGAAGAAGACTGCCGAGGCTTTCCTTGAACCG 414 GAGATCTCCTCAATGGTTTTGCTGAAGATGAAGGAGACTGCCGAGGCTTTCCTTGGAACC 414 GAGATCTCCTCAATGGTGCTTATTAAGATGAAAGAAACTGCAGAAGCCTTTTTGGGTGGT 411 GAGATCTCCTCAATGGTGCTTATTAAGATGAAAGAAACTGCAGAAGCCTTTTTGGGTGGT 411 GAAATTTCATCAATGGTGCTGGTAAAAATGAAAGAAACTGCAGAAGCATATCTAGGAAAG 414 GAGATCTCCTCCATGGTATTGGTTAAGATGAAGGAAACAGCCGAGGCTTACATCGGCAAG 408 GAAATTTCCTCCATGGTACTTTTGAAAATGAAAGAAACTGCTGAAGCCTATCTTGGAAAG 411 GAGATTTCCTCCATGGTGCTAACAAAGATGAAGGAAACTGCAGAACAGTATTTGGGAAAG 411 GAAATTTCATCTATGGTTCTGGTCAAGATGAAGGAGATTGCTGAGGCCTACCTTGGGAAA 411 GAGATCTCCTCCATGGTGCTGCTGAAGATGAAGGAGATTGCTGAGGCATACCTTGGCAAG 411 GAGGTGTCTTCTATGGTTCTGACAAAGATGAAGGAAATTGCAGAAGCCTACCTTGGGAAG 411 GAAGTGTCTTCAATGGTTCTGACAAAAATGAAGGAAATTGCAGAAGCTTACCTTGGAAAG 411 GAAATATCTTCCATGGTTTTGACAAAGATGAAAGAGATAGCAGAAGCATATCTTGGGAAG 411 GAGATCTCGTCGATGGTGCTGACCAAGATGAAGGAGATCGCCGAGGCGTACCTGGGCCAC 411 GAGATCTCGTCCATGGTGCTGACCAAGATGAAGGAGATCGCCGAGGCGTACCTGGGCTAC 411 GAGATCTCGTCCATGGTGCTGACCAAGATGAAGGAGATCGCCGAGGCGTACCTGGGCCAC 411 GAGATTTCCTCGATGGTCCTGGTTAAAATGAGAGAAATAGCTGAGGCCTTTCTGGGACAG 417 GAGATCTCCTCCATGGTCCTGGTGAAGATGAAGGAAATCGCCGAGGCCTACCTGGGCCAC 417 GAAATCTCTTCGATGGTATTGACTAAGTTGAAGGAGACTGCTGAGGCCTTTTTGGGCCAC 417 GAGATCTCGTCCATGGTGCTGAGCAAGATGAAGGAGACGGCCGAGGCGTACCTGGGCCAG 417 GAGATCAGCTCCATGGTGCTGACTAAGATGAAGGAGATCGCCGAAGCCTACCTGGGCCGC 414

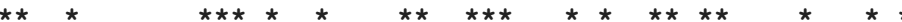

AGCATCACGGATGCAGTCATCACAGTTCCAGCTTACTTCAACGACTCTCAGCGCCAGGCT 462 AGCATCACAGACGCAGTCATCACAGTTCCAGCCTACTTCAACGACTCCCAGCGCCAGGCT 462 ACAGTACGCGACGCAGTGATCACAGTGCCGGCGTACTTCAACGACTCTCAGCGCCAGGCC 462 ACAGTGCGGGATGCGGTAGTCACAGTTCCGGCATACTTCAACGACTCCCAGCGTCAGGCC 462 AAAGTGTTAGATGCCGTCATAACTGTCCCCGCTTACTTCAACGACTCTCAGAGACAAGCT 477 AAGGTCACGGACGCAGTGGTGACCGTGCCCGCCTACTTCAACGACTCGCAGCGCCAAGCG 471 
bioRxiv preprint doi: https://doi.org/10.1101/2020.09.21.307264; this version posted April 22, 2021. The copyright holder for this preprint (which was not certified by peer review) is the author/funder, who has granted bioRxiv a license to display the preprint in perpetuity. It is made available under aCC-BY-NC-ND 4.0 International license.

B plicatilis HSP70-2 AB775785

A_brightwelli__HSP70_KX119429

Wuchereria AF167352

Setaria_AF $\overline{0} 79360$

C elegañs HSP70A M1 8540

C_elegans_HSP-1_NM_070667

M_nipponense_KC $\overline{4} 60 \overline{3} 43$

M_nipponense_DQ660140

Mýtilus_HSP70_AY861684

Perinereis_KU $\overline{2} 55783$

Paralvinelīa_HSP70-2_EF580993

Haliotis FJ81̄2176

Oncorhynchus_HSC71_AAB21658

$\mathrm{X}$ maculatus $\overline{\mathrm{H} S C} 70$ AB062115

Human_HSPA8_HSC71_NP_006588

Rattus HSC7 $\overline{1}$ NM $0 \overline{2} 43 \overline{5} 1$

Pelodiscus_HS̄C70̄HQ219723

BOS_HSP70_Ū09861

Humān_HSPĀ1A_NM_005345

Rattus_HSP70_1A_NM_031971

$\mathrm{X}$ maculatus $\overline{\mathrm{H} S P} \overline{7} \mathrm{O} \overline{\overline{1}}$ AB0 62113

X_maculatus_HSP70-2_AB062114

Human HSPA1 $\overline{\mathrm{L}}$ NM $005 \overline{5} 27$

Human_HSPA 6 N $P$ - 002146

Alligätor_HS̄P70_AB306279

Drosophila_Bb_AF2 95957

Drosophila_Aa_AF295933

Spodoptera_FJ $\overline{7} 54276$

Bombyx ABO $\overline{3} 5326$

Paralvinella EF580992

B_plicatilis_HSP70-1_AB775784

B plicatilis HSP70-2 AB775785

A_brightwellī_HSP70_KX119429

Wüchereria AF'167352

Setaria_AF079360

C_elegañs_HSP70A_M18540

C-elegans HSP-1 $\overline{\mathrm{N} M} 070667$

M_nipponense_KC $\overline{4} 60 \overline{3} 43$

M_nipponense_DQ660140

Mýtilus_HSP70_AY861684

Perinerēis KU $\overline{2} 55783$

Paralvinelīa_HSP70-2_EF580993

Haliotis FJ812176

Oncorhynchus_HSC71_AAB21658

$X$ maculatus $\bar{H} S C 70$ AB 062115

Human_HSPA8_HSC71_NP_006588

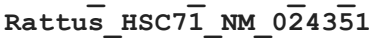

Pelodiscus_HS̄C70_HQ219723

Bos HSP7O Ū09861

Humān HSPĀ1A NM 005345

Rattus HSP70_1A-NM 031971

$X$ maculatus $\bar{H} S P \overline{7} 0-\overline{1}$ ABO 62113

X_maculatus_HSP70-2_AB0 62114

Human HSPA1 $\overline{\mathrm{L}}$ NM $005 \overline{5} 27$

Human_HSPA6_NP_ 002146

Alligätor_HS̄P7O_AB306279

Drosophila_Bb_AF295957

Drosophila_Aa_AF295933

Spodoptera FJ754276

Bombyx_AB0 35326

Paralvinella EF580992

B_plicatilis_HSP70-1_AB775784

B plicatilis HSP70-2 AB775785

A_brightwellí_HSP70_KX119429

Wüchereria AF'167352

Setaria_AF $\overline{0} 79360$

C_elegañs_HSP70A_M18540

C-elegans_HSP-1_NM_070667

M_nipponense_KC $\overline{4} 60 \overline{3} 43$

Mnipponense DQ660140

Mytilus_HSP70_AY861684

Perinerēis KU 255783

Paralvinelīa_HSP70-2_EF580993

Haliotis_FJ812176

Oncorhynchus_HSC71_AAB21658
AAGGTCACGGACGCAGTGGTGACCGTGCCCGCCTACTTCAACGACTCGCAGCGCCAAGCG 471 AAAGTAAACGACGCCGTGATCACAGTCCCGGCCTATTTCAACGACTCTCAAAGACAGGCC 468 GCGGTCAAGGATGCTGTTATTACAGTCCCCGCATATTTTAATGACTCACAGCGACAAGCC 471 GCAGTCAAGGATGCTGTGATCACAGTTCCAGCTTATTTCAATGACTCACAGCGACAGGCA 471 ACCGTCAAGGATGCCGTTGTCACTGTCCCGACTTACTTCAACGACTCGCAGCGTCAAGCC 474 ACCGTCAAGGATGCCGTTGTCACTGTCCCAGCTTACTTCAACGACTCGCAGCGTCAAGCC 474 ACTGTAAAGGATGCTGTCGTCACAGTCCCTGCCTATTTCAATGACTCCCAGCGTCAGGCC 471 ACTGTAAAGGATGCTGTCGTCACAGTCCCTGCCTATTTCAATGACTCCCAGCGTCAGGCC 471 TTAGTCAACAACAGTGTCATTACAGTCCCAGCTTATTTTAATGATTCACAAGGACAAGCA 474 ACTGTTCTAAATGCCGTCGTAACAGTGCCTGCCTACTTCAACGATTCTCAGCGCCAAGCA 468 AACGTACAGAGCGCTGTGATCACAGTACCAGCCTATTTCAATGACAGCCAGCGTCAGGCC 471 ACTATAACAGACGCTGTTGTAACAGTCCCAGCTTACTTCAACGACTCTCAGCGACAGGCC 471 ACTGTCAACAATGCTGTTGTTACCGTACCTGCCTACTTCAATGACTCCCAGCGCCAGGCA 471 ACTATAACAAATGCCGTGGTGACTGTACCTGCCTACTTCAATGACTCTCAGCGTACGGCC 471 ACTGTTACCAATGCTGTGGTCACAGTGCCAGCTTACTTTAATGACTCTCAGCGTCAGGCT 471 ACTGTTACCAATGCCGTGGTCACCGTGCCAGCTTACTTCAATGACTCTCAGCGACAGGCA 471 ACTGTTACTAATGCAGTGGTCACGGTACCAGCCTACTTCAATGACTCCCAGCGCCAGGCC 471 CCGGTGACCAACGCGGTGATCACCGTGCCGGCCTACTTCAACGACTCGCAGCGGCAGGCC 471 CCGGTGACCAACGCGGTGATCACCGTGCCGGCCTACTTCAACGACTCGCAGCGCCAGGCC 471 CCGGTGACCAACGCGGTGATCACCGTGCCCGCCTACTTCAACGACTCGCAGCGGCAGGCC 471 AGGGTGTCGAATGCGGTCATCACAGTGCCAGCTTACTTTAATGATTCCCAAAGGCAAGCC 477 AAGGTGTCCAACGCCGTGATCACGGTCCCCGCCTATTTCAACGACTCCCAGCGACAGGCC 477 CCTGTCACCAATGCAGTGATTACCGTGCCAGCCTATTTCAATGACTCTCAACGTCAGGCT 477 CCCGTGAAGCACGCAGTGATCACCGTGCCCGCCTATTTCAATGACTCGCAGCGCCAGGCC 477 AAGGTGCAGAACGCCGTCATCGCGGTGCCCGCCTACTTCAACGACTCGCAGCGCCAGGCC 474

ACCAAAGACGCCGGTCACATCGCCGGCCTGAATGTGCTCCGCATCATCAATGAGCCCACG 522 ACCAAAGACGCCGGTCACATCGCCGGCCTGAATGTGCTCCGCATCATCAATGAGCCCACG 522 ACCAAGGACGCGGGAGCCATCGCCGGGCTGAACGTGCTCAGGATCATCAACGAGCCCACA 522 ACCAAGGACGCCGGAGCCATCGCCGGCCTGAACGTGCTTCGCATCATCAACGAGCCCACA 522 ACAAAAGATGCTGGTCTCATCTCCGGATTGAACGTTCTCCGAGTGATTAACGAGCCAACA 537 ACCAAGGACGCGGGCGCCATCGCCGGCCTCAACGTGCTGCGCATCATCAACGAGCCCACT 531 ACCAAGGACGCGGGCGCCATCGCCGGCCTCAACGTGCTGCGCATCATCAACGAGCCCACT 531 ACAAAAGACGCCGGCGCCATTTCAGGTCTGAACGTGTTACGTATCATAAACGAGCCAACA 528 ACTAAAGATTCCGGTGCAATTGCTGGTCTAAATGTCTTGCGTATTATCAACGAACCAACA 531 ACGAAAGATTCGGGTGCCATTGCGGGTTTGAACGTTCTGCGTATTATCAACGAACCAACC 531 ACCAAGGATGCCGGAGCCATCGCTGGACTCAACGTTCTCCGTATCATCAACGAGCCAACC 534 ACCAAGGATGCCGGAGCCATCGCTGGACTCAACGTTCTCCGTATCATCAACGAGCCAACC 534 ACCAAAGATGCTGGTACAATCTCTGGTCTCAATGTGCTCCGTATCATCAATGAGCCCACT 531 ACCAAAGATGCTGGTACAATCTCTGGTCTCAATGTGCTCCGTATCATCAATGAGCCCACT 531 ACAAAGGATGCTGGTACCATCTCTGGAATGAATGTTCTACGTATTATCAATGAGCCAACT 534 ACCAAAGATGCCGGAACTATCTCTGGCTTGAATGTATTACGTATCATCAATGAACCAACG 528 ACCAAAGATGCTGGTACCATCTCTGGTATGAATGTGCTGCGTATCATCAATGAGCCCACA 531 ACTAAAGATGCAGGGACAATCTCTGGTCTCAATGTTTTACGTATCATCAATGAGCCTACT 531 ACCAAAGATGCTGGTACCATCTCGGGGCTGAATGTGCTGCGTATCATCAATGAGCCAACT 531 ACCAAAGACGCTGGGACCATTTCTGGGCTCAATGTTCTGCGTATCATTAACGAGCCAACG 531 ACCAAAGATGCTGGAACTATTGCTGGTCTCAATGTACTTAGAATTATTAATGAGCCAACT 531 ACAAAAGATGCTGGAACTATTGCTGGCCTCAACGTACTTCGAATTATCAATGAGCCAACT 531 ACAAAAGATGCTGGAACCATTGCAGGTCTTAATGTGCTCAGGATCATCAATGAACCAACT 531 ACCAAGGACGCGGGGGTGATCGCGGGGCTGAACGTGCTGAGGATCATCAACGAGCCCACG 531 ACCAAGGATGCGGGTGTGATCGCGGGGCTCAACGTGCTGCGGATCATCAACGAGCCCACG 531 ACCAAGGACGCGGGCGTGATCGCGGGTCTGAACGTGCTGCGGATCATCAACGAGCCCACG 531 ACAAAGGATGCTGGAGTGATCTCCGGACTAAATGTTCTCCGCATCATAAATGAGCCGACA 537 ACCAAAGACGCAGGCGTCATTGCGGGACTCAACGTCTTGAGGATCATCAACGAGCCCACG 537 ACTAAGGATGCAGGTGTGATTGCTGGACTTAATGTGCTAAGAATCATCAATGAGCCCACG 537 ACCAAGGACGCGGGGGCCATCGCGGGGCTCAACGTGTTGCGGATCATCAATGAGCCCACG 537 ACCAAGGACGCGGGCACCATCACGGGCCTCAACGTGATGCGCATCATCAACGAGCCCACG 534

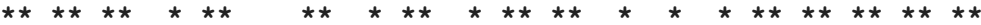

GCGGCAGCATTGGCCTACGGACTGGACAAGAACCTCAA------GGGTGAGCGCAATGTG 576 GCGGCAGCACTGGCCTACGGACTGGACAAGAACCTCAA-------GGGTGAGCGCAATGTG 576 GCCGCTGCGCTCGCCTACGGCCTCGACAAGAACCTCAA------GGGCGAGAGGAACGTC 576 GCCGCCGCGCTCGCGTACGGTCTCGACAAGAACCTCAA------GGGTGAGAGAAATGTT 576 GCAGCCGCTCTGGCTTATGGCTTGGATAAGAACCTATC------TGGAGAGAAGAACGTC 591 GCGGCCGCCATCGCCTACGGCCTTGACAAAAAGGGCGG------CGGCGAAAAAAACATC 585 GCGGCCGCCATCGCCTACGGCCTTGACAAAAAGGGCGG------CGGCGAAAAAAACATC 585 GCAGCTGCGATCGCTTACGGTCTTGATAAAAAAGTCGG------CGGTGAGAAAAACGTG 582 GCAGCTGCTATTGCTTATGGTTTGGACAAGAAGGGCCA------TGGTGAACGCAACGTT 585 GCAGCAGCTATTGCATATGGGTTGGACAAGAAGGGCCA------TGGTGAGCGTAATGTC 585 GCTGCAGCTATCGCTTACGGACTTGACAAGAAGGGACA-------CGGAGAACGCAACGTT 588 GCTGCAGCTATCGCTTACGGACTTGACAAGAAGGGACA------CGGAGAACGCAACGTT 588 GCTGCTGCCATCGCATATGGGCTGGACAAGAAAGTTGG------TGGAGAACGTAATGTT 585 GCTGCTGCCATCGCATATGGGCTGGACAAGAAAGTTGG--_----TGGAGAACGTAATGTT 585 GCAGCTGCTATTGCTTATGGTTTGGATAAGAAAGTAGG------TGGAGAAAGAAATGTA 588 GCCGCCGCCATCGCTTACGGTCTTGATAAGAAGGTGGG------AGGAGAGAGAAATGTC 582 GCTGCCGCCATTGCTTACGGTTTGGACAAGAAGGTTGG------TGGAGAGCGTCATGTG 585 GCCGCAGCTATTGCATACGGTCTTGACAAGAAGGTTGG------TGGTGAACGCAACGTT 585 GCTGCTGCCATTGCCTACGGCCTGGACAAGAAGGTCGG------TGCTGAAAGGAATGTC 585 
bioRxiv preprint doi: https://doi.org/10.1101/2020.09.21.307264; this version posted April 22, 2021. The copyright holder for this preprint (which was not certified by peer review) is the author/funder, who has granted bioRxiv a license to display the preprint in perpetuity. It is made available under aCC-BY-NC-ND 4.0 International license.

$X$ maculatus HSC70 AB062115 Human_HSPA8 HSC71_NP 006588 Rattus HSC7 $\overline{1}$ NM $0 \overline{2} 43 \overline{5} 1$

Pelodiscus_HS̄C70̄HQ219723 Bos HSP70 Ū09861

Humān_HSPĀ1A_NM_005345 Rattus HSP70 1A NM 031971

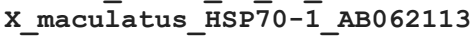
X_maculatus_HSP70-2_AB062114 Hüman HSPA1 $\overline{\mathrm{L}}$ NM $005 \overline{5} 27$ Human_HSPA $6 \overline{\mathrm{N} P} \overline{0} 02146$ Alligātor_HS̄P70_AB306279

Drosophila_Bb_AF2 95957 Drosophila_Aa_AF295933 Spodoptera_FJ754276 Bombyx_AB0 35326 Paralvinella_EF580992 B_plicatilis_HSP70-1_AB775784 B_plicatilis_HSP70-2_AB775785 A_brightwellī_HSP70_K̄X119429 Wüchereria AF'167352 Setaria_AF079360 C_elegañs_HSP70A_M1 8540 C_elegans_HSP-1_NM_070667 Mnipponense_KC $\overline{4} 60 \overline{3} 43$ M nipponense DQ660140 Mýtilus_HSP70_AY861684 Perinereis KU 255783 Paralvinelīa_HSP70-2_EF580993 Haliotis FJ81̄2176 Oncorhynchus_HSC71_AAB21658 $x$ maculatus $\bar{H} S C 70$ AB062115 Human_HSPA8_HSC71_NP_006588 Rattus HSC7 $\overline{1}$ NM $0 \overline{2} 43 \overline{5} 1$ Pelodiscus_HS̄C70̄HQ219723 Bos_HSP70 Ū09861

Human_HSPĀ1A_NM_005345 Rattus_HSP70_1A_NM_031971

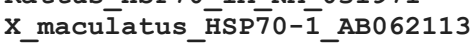
X_maculatus_HSP70-2_AB062114 Human HSPA1 $\overline{\mathrm{L}}$ NM $005 \overline{5} 27$ Human_HSPA 6 N $P$ - 002146 Alligätor_HS̄P70_AB306279

Drosophila_Bb_AF2 95957 Drosophila_Aa_AF2 95933 Spodoptera_FJ754276 Bombyx ABO $\overline{3} 5326$ Paralvinella EF580992 B_plicatilis_HSP70-1_AB775784 B plicatilis HSP70-2 AB775785 A_brightwellī_HSP70_KX119429 Wüchereria AF'167352 Setaria_AF 079360 C_elegañs_HSP70A_M1 8540 C_elegans_HSP-1_NM_070667 Mnipponense_KC $\overline{4} 60 \overline{3} 43$ M nipponense DQ660140 Mýtilus_HSP70_AY861684 Perinerēis KU 255783 Paralvinelīa_HSP70-2_EF580993 Haliotis FJ812176 Oncorhynchus_HSC71_AAB21658 $x$ maculatus_HSC70 ÄB062115 Human_HSPA8 HSC71-NP_006588 Rattus_HSC7 $\overline{1}$ NM $0 \overline{2} 43 \overline{5} \overline{1}$ Pelodiscus_HS̄C70_HQ219723 Bos HSP70 Ū09861 Humān_HSPĀ 1 A_NM_005345 Rattus_HSP70_1A_NM_031971

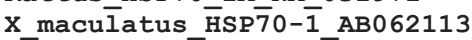
X_maculatus_HSP70-2_AB062114 Human HSPA1 $\overline{\mathrm{L}}$ NM $005 \overline{5} 27$ Human HSPA $6 \overline{\mathrm{N} P} \overline{0} 02146$ Alligātor_HS̄PTO_AB306279
GCTGCTGCCATTGCTTACGGTTTGGACAAAAAGGTCGG------GTGCGAGAAAAACGTC 585 GCTGCTGCTATTGCTTACGGCTTAGACAAAAAGGTTGG------AGCAGAAAGAAACGTG 585 GCTGCTGCTATTGCCTATGGCTTAGATAAGAAGGTCGG------GGCTGAAAGGAATGTG 585 GCTGCTGCTATTGCTTATGGCTTGGACAAGAAGGTTGG------AGCGGAAAGGAATGTC 585 GCCGCCGCCATCGCCTACGGCCTGGACAGGACGGGCAA------GGGGGAGCGCAACGTG 585 GCCGCCGCCATCGCCTACGGCCTGGACAGAACGGGCAA-------GGGGGAGCGCAACGTG 585 GCGGCCGCCATCGCCTACGGGCTGGACCGGACCGGCAA------_GGGCGAGCGCAACGTG 585 GCTGCAGCCATTGCCTACGGCCTTGATAAAGGCAAAAG------AGGAGAGCGCAATGTC 591 GCGGCCGCCATCGCCTACGGTCTGGACAAAGGCAAGTC------GGGAGAGAGAAACGTC 591 GCTGCTGCCATTGCCTATGGTTTAGATAAAGGAGGTCA------AGGAGAACGACATGTC 591 GCAGCTGCCATCGCCTATGGGCTGGACCGGCGGGGCGC-------GGGAGAGCGCAACGTG 591 GCGGCCGCCATCGCCTACGGGCTGGACAAGAAGGGCGCGCGCGCCGGCGAGAAGAACGTG 594

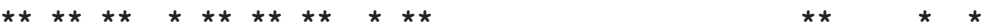

СTTATCCTCGACTTGGGCGGCGGCACCTTCGATGTCTCCATCCTGACCATCGACGAGGGA 636 СTTATCTTCGACTTGGGCGGCGGCACCTTCGATGTCTCCATCCTGACCATCGACGAGGGA 636 CTCATTTTCGACCTCGGCGGCGGAACGTTCGACGTGTCGATCCTGACCATCGACGAAGGT 636 CTTATCTTCGATCTGGGTGGAGGAACCTTCGACGTGTCCATCCTGACTATCGACGAGGGA 636 TTGATATTTGACCTCGGAGGAGGAACGTTCGATGTGTCGATTCTCTCGATTGCCGATGGT 651 СTGATCTTTGACCTGGGCGGCGGCACCTTCGACGTCTCCATCCTGACCATCGAAGAGGG- 644 СTGATCTTTGACCTGGGCGGCGGCACCTTCGACGTCTCCATCCTGACCATCGAAGAGGG- 644 TTAATCTTCGACTTGGGCGGCGGTACTTTCGATGTGTCGATCTTGACCATTGAAGAAGG- 641 СTTATCTTTGATCTTGGTGGTGGTACATTTGATGTGTCTATCCTAACTATCGAGGATGG- 644 СTTATATTTGACCTTGGTGGTGGCACATTTGATGTGTCTATCCTGACTATAGAGGATGG- 644 СTTATCTTCGATCTTGGAGGTGGTACCTTCGATGTCTCCATTCTTACCATTGAGGACGG- 647 СTTATCTTCGATCTTGGAGGTGGTACCTTCGATGTCTCCATTCTTACCATTGAGGACGG- 647 CTGATTTTTGATTTAGGTGGTGGCACTTTTGATGTATCTATTCTAACTATTGAAGATGA- 644 CTGATTTTTGATTTAGGTGGTGGCACTTTTGATGTATCTATTTTAACTATTGAAGATGG- 644 CTCATCTTTGACTTGGGTGGTGGAACTTTTGATGTGTCAATCCTTACAATTAAGGATGG- 647 CTGATTTTTGATCTCGGTGGTGGAACCTTCGATGTGTCCATCTTGACCATTGAGGATGG- 641 CTGATCTTCGATCTGGGTGGAGGCACCTTTGATGTGTCCGTTCTGACCATAGAGGATGG- 644 СTCATCTTTGATCTTGGCGGTGGTACATTTGATGTGTCTATCTTGACAATCGAAGATGG- 644 СTTATCTTTGATCTGGGTGGCGGCACCTTTGACGTGTCCATCTTGACCATCGAGGATGG- 644 СTCATCTTCGACCTGGGCGGCGGCACTTTCGACGTCTCCATCCTGACCATCGAGGACGG- 644 СTCATCTTTGACCTGGGAGGTGGCACTTTTGATGTGTCAATCCTCACTATTGAGGATGG- 644 CTCATTTTTGACTTGGGAGGTGGCACTTTTGATGTGTCAATCCTCACTATCGAGGATGG- 644 СTTATCTTTGACCTTGGGGGTGGCACTTTCGATGTTTCAATCCTCACTATTGAGGATGG- 644 CTCATCTTTGATCTGGGAGGGGGCACGTTCGACGTGTCCATCCTGACGATCGACGACGG- 644 СTCATCTTTGACCTGGGCGGGGGCACCTTCGACGTGTCCATCCTGACGATCGACGACGG- 644 CTCATCTTCGACCTGGGGGGCGGCACGTTCGACGTGTCCATCCTGACGATCGACGACGG- 644 СTCATCTTTGATCTTGGTGGAGGCACTTTTGACGTGTCCATCCTGACCATTGAGGATGG- 650 CTGATCTTTGACCTGGGCGGAGGCACCTTCGACGTGTCCGTCCTGACCATCGAGGACGG- 650 CTGATTTTTGATCTGGGTGGAGGCACATTTGATGTGTCAATTCTGACCATAGATGATGG- 650 СTCATTTTTGACCTGGGTGGGGGCACCTTCGATGTGTCGGTTCTCTCCATTGACGCTGG- 650 CTGATCTTCGACCTGGGCGGCGGCACCCTCGACGTGTCCATCCTCACCATCGAGGACGG- 653

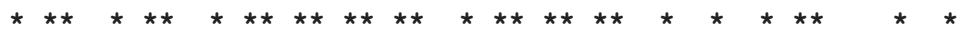

TCTCTGTTCGAGGTGCGCTCCACAGCCGGAGACACACACTTGGGCGGCGAGGACTTTGAC 696 TCTCTGTTCGAGGTGCGCTCCACCGCCGGAGACACGCACTTGGGCGGCGAGGACTTTGAC 696 TCATTGTTCGAAGTGAGGGCGACGGCGGGCGACACGCACCTCGGAGGCGAGGACTTCGAC 696 TCGTTGTTCGAAGTGAAGTCCACAGCCGGCGACACGCACCTGGGAGGCGAAGACTTTGAT 696 TCGCTGTTCGAGGTGAAGTCGACGGCCGGAGACACGCATCTCGGTGGCGAGGACTTCGAC 711 --CATCTTCGAGGTCAAGTCCACTGCCGGCGACACTCATTTAGGCGGCGAGGACTTCGAC 702 --CATCTTCGAGGTCAAGTCCACTGCCGGCGACACTCATTTAGGCGGCGAGGACTTCGAC 702 --TATCTTCGAGGTAAAATCCACGGCCGGAGACACACATTTGGGCGGCGAGGACTTTGAT 699 --TATTTTCGAAGTCAAATCTACAGCTGGAGATACTCATCTTGGAGGAGAAGATTTCGAT 702 --TATTTTCGAAGTGAAATCTACTGCTGGTGACACTCATCTTGGAGGCGAAGATTTCGAT 702 --AATCTTCGAAGTCAAGTCTACCGCTGGAGACACTCATCTTGGAGGAGAGGACTTCGAT 705 --AATCTTCGAAGTCAAGTCTACCGCTGGAGACACTCATCTTGGAGGAGAGGACTTCGAT 705 --CATTTTTGAAGTGAAGTCTACTGCTGGTGACACACATTTAGGTGGTGAAGATTTTGAT 702 --CATTTTTGAAGTGAAGTCTACTGCTGGTGACACACATTTAGGTGGTGAAGATTTTGAT 702 --TATTTTTGAAGTTAAATCAACCTCTGGTGATACCCACTTGGGTGGTGAAGACTTTGAC 705 --TATCTTTGAAGTGAAGTCAACAGCCGGAGACACCCATTTGGGAGGAGAGGATTTCGAC 699 --CATCTTTGAAGTGAAATCCACATCCGGTGACACTCACTTGGGTGGTGAGGACTTCGAC 702 --TATCTTTGAGGTGAAATCTACAGCTGGAGACACCCACTTGGGTGGTGAGGACTTTGAC 702 --СATCTTTGAGGTCAAGTCCACTGCTGGAGACACTCATCTGGGTGGAGAAGACTTTGAC 702 --CATCTTTGAGGTGAAGGCCACGGCGGGCGACACCCACCTGGGCGGCGAGGACTTCGAC 702 --AATCTTTGAGGTCAAGTCTACAGCTGGAGACACCCACTTGGGTGGAGAAGATTTTGAC 702 --AATTTTTGAAGTCAAATCAACAGCTGGAGACACCCACTTGGGCGGAGAAGACTTTGAC 702 --CATCTTTGAAGTGAAGTCAACTGCTGGTGATACCCACTTAGGTGGGGAGGACTTTGAT 702 --CATCTTCGAGGTGAAGGCCACGGCCGGGGACACGCACCTGGGCGGGGAGGACTTCGAC 702 --CATCTTCGAGGTGAAGGCCACGGCCGGGGACACCCACCTGGGTGGGGAGGACTTTGAC 702 --CATCTTCGAGGTGAAGGCCACGGCGGGCGACACGCACCTGGGCGGGGAGGACTTCGAC 702 --CATATTTGAGGTGAAATCCACGGCCGGGGACACACATCTCGGCGGAGAGGATTTTGAC 708 --СATCTTTGAGGTGAAGGCCACGGCCGGAGACACTCACTTGGGAGGAGAGGACTTTGAC 708 --GATTTTTGAGGTAAAGGCCACTGCTGGGGACACTCACCTGGGTGGGGAGGACTTTGAC 708 --TGTCTTTGAGGTGAAAGCCACTGCTGGAGATACCCACCTGGGAGGAGAGGACTTCGAC 708 --CATCTTCGAGGTGAAGTCGACGGCGGGCGACACGCACCTGGGCGGCGAGGACTTCGAC 711

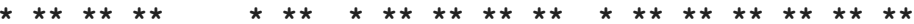


bioRxiv preprint doi: https://doi.org/10.1101/2020.09.21.307264; this version posted April 22, 2021. The copyright holder for this preprint (which was not certified by peer review) is the author/funder, who has granted bioRxiv a license to display the preprint in perpetuity. It is made available under aCC-BY-NC-ND 4.0 International license.

Drosophila_Bb_AF295957 Drosophila Aa-AF295933 Spodoptera_FJ754276 Bombyx AB0 $\overline{3} 5326$

Paralvinella_EF580992 B_plicatilis_HSP70-1_AB775784 B plicatilis HSP70-2 AB775785 A_brightwellī_HSP70_KX119429 Wüchereria AF'167352 Setaria_AF $\overline{0} 79360$ C elegañs HSP70A M1 8540 C_elegans_HSP-1_N̄M_070667 M nipponense KC4 $60 \overline{3} 43$ M_nipponense_DQ660140 Mytilus HSP70 AY 861684 Perinereis KU $\overline{2} 55783$ Paralvinelīa_HSP70-2_EF580993 Haliotis_FJ812176 Oncorhynchus_HSC71_AAB21658 $X$ maculatus $\overline{\mathrm{H} S C 70} \overline{\mathrm{A}} \mathrm{B} 062115$ Hüman_HSPA8_HSC71_NP_006588 Rattus $\bar{s}$ HSC $\overline{1}$ NM $0 \overline{2} 43 \overline{5} 1$

Pelodiscus_HS̄C70̄HQ219723 BOS HSP70 Ü09861

Humān_HSPĀ1A_NM_005345 Rattus HSP70_1A-NM 031971 $X$ maculatus_ $\bar{H} S P \overline{7} 0-\overline{1}$ _AB0 62113 X_maculatus_HSP70-2_AB0 62114 Human_HSPA1 $\overline{\mathrm{L}}$ NM $005 \overline{5} 27$ Human HSPA $6 \overline{\mathrm{N} P} \overline{0} 02146$ Alligätor_HS̄P70_AB306279

Drosophila_Bb_AF2 95957 Drosophila Aa-AF295933 Spodoptera_FJ754276 Bombyx_AB0 $\overline{3} 5326$

Paralvinella_EF580992 B_plicatilis_HSP70-1_AB775784 B_plicatilis_HSP70-2_AB775785 A_brightwellii_HSP70_KX119429 Wüchereria AF'167352 Setaria_AF $\overline{0} 79360$ C elegañs HSP70A M18540 C_elegans_HSP-1_NM_070667 Mnipponense_KC $\overline{4} 60 \overline{3} 43$ M_nipponense_DQ660140 Mýtilus_HSP70 AY861684 Perinereis KU 255783 Paralvinelīa_HSP70-2_EF580993 Haliotis FJ81̄2176 Oncorhynchus_HSC71_AAB21658 $\mathrm{X}$ maculatus_HSC70_A $\mathrm{A} 062115$ Human_HSPA8_HSC71_NP_006588 Rattus $\bar{s}$ HSC7 $\overline{1}$ NM $0 \overline{2} 43 \overline{5} 1$ Pelodis̄cus_HS̄C70̄HQ219723 Bos_HSP70 Ū09861

Human_HSPĀ1A_NM_005345 Rattus_HSP70_1A_NM_031971 X_maculatus_ $\bar{H} S P \overline{7} 0-\overline{1}$ _AB0 62113 X_maculatus_HSP70-2_AB062114 Human HSPA $1 \overline{\mathrm{L}}$ NM $005 \overline{5} 27$ Human HSPA 6 N P $\overline{0} 02146$ Alligator_HSיPTO_AB306279

Drosophila_Bb_AF295957 Drosophila_Aa_AF295933 Spodoptera FJ $\overline{7} 54276$ Bombyx_AB0 35326 Paralvinella EF580992 B_plicatilis_HSP70-1_AB775784 B plicatilis HSP70-2 AB775785 A_brightwellìi_HSP70_KX119429 Wüchereria AF'167352 Setaria_AF 079360 C_elegañs_HSP70A_M18540 C_elegans_HSP-1_NM_070667
AACCGGCTAGTCACCCACCTGGCGGAGGAGTTCAAGCGCAAGTACAAGAAGGATCTGCGC 756 AACCGGCTAGTCACTCATCTGGCGGACGAGTTCAAGCGCAAGTACAAGAAGGATCTGCGC 756 AACAGATTGGTGAACCATCTCGCGGACGAGTTCAAGCGCAAGTACAAGAAGGACATGCGC 756 AACCGACTAGTCAACCATCTTGCGGAAGAGTTCAAGCGCAAGTACAAGAAGGATCTGCGC 756 AACAGACTGGTGAACCATTTCATCAAGGAATTCTTTAGAAAGTACAAGAAAGACATCAGC 771 AACCGACTGGTCAACCATTTTGTCGAGGAGTTCAAGCGCAAGAACAAAAAGGACATCACC 762 AACCGACTGGTCAACCATTTTGTCGAGGAGTTCAAGCGCAAGAACAAAAAGGACATCACC 762 AACAGACTCGTGAACCATTTCGTCGAAGAATTCAAGAGAAAACATAAAAAAGACATCACC 759 AATCGCATGGTGAATCATTTCGTTGCGGAATTCAAGCGGAACGACAAAAAGGATCTTGCG 762 AACCGCATGGTGAATCATTTCGTTGCGGAATTCAAGCGTAAACACAAGAAGGATTTGGCT 762 AACCGCATGGTGAACCACTTCTGTGCCGAGTTCAAGCGCAAGCACAAGAAGGATCTTGCT 765 AACCGCATGGTGAACCACTTCTGTGCCGAGTTCAAGCGCAAGCACAAGAAGGATCTTGCT 765 AACAGAACGGTTAACCATTTCATTCAAGAATTCAAGAGAAAATACAAGAAAGATCCTTCC 762 AACAGAATGGTTAACCATTTCATTCAAGAATTCAAGAGAAAATACAAGAAAGATCCTTCC 762 AACAGAATGGTCAATCATTTCATTCAAGAATTCAAACGCAAGCACAAAAAAGACATTAGT 765 AACAGAATGGTCAACCACTTCATTCAGGAATTCAAGCGCAAGTTCAAGAAAGACATCTCT 759 AACCGTATGGTGAACCACTTCATTCAGGAATTTAAGAGAAAGTTCAAGAAGGACATCTCC 762 AATCGTATGGTGAACCACTTCATCCAAGAGTTCAAAAGGAAACACAAGAAGGATATCTCT 762 AACCGCATGGTCAACCACTTCATCGCGGAGTTCAAACGCAAGTACAAGAAAGACATCAGC 762 AACCGCATGGTGAACCACTTCATCTCCGAGTTCAAGCGCAAGTACAAGAAGGACATCAGC 762 AACCGAATGGTCAACCATTTTATTGCTGAGTTTAAGCGCAAGCATAAGAAGGACATCAGT 762 AACCGAATGGTCAACCATTTCATTGCTGAGTTTAAGCGAAAGCACAAGAAGGACATCAGT 762 AACCGTATGGTCAATCATTTCATTGCTGAATTCAAGCGTAAGCACAAGAAGGATATTACT 762 AACAGGCTGGTGAACCACTTCGTGGAGGAGTTCAAGAGGAAGCACAAGAAGGACATCAGC 762 AACAGGCTGGTGAACCACTTCGTGGAGGAGTTCAAGAGAAAACACAAGAAGGACATCAGC 762 AACCGGCTGGTGAGCCACTTCGTGGAGGAGTTCAAGAGGAAGCACAAGAAGGACATCAGC 762 AACCGAATGGTCAGCCACTTTGTGGAGGAGTTTAAAAGAAAGCACAAGAAGGACATCAGC 768 AACCGCATGGTCAACCACTTTGTGGAGGAGTTCAAGAGGAAACACAAGAAGGACATCAGC 768 AACAGGCTTGTGAGCCACTTCGTGGAGGAGTTCAAGAGGAAACACAAAAAGGACATCAGC 768 AACCGGCTCGTGAACCACTTCATGGAAGAATTCCGGCGGAAGCATGGGAAGGACCTGAGC 768 AACCGCATGGTGACGCACTTCGTGGAGGAGTTCAAGCGCAAGCACAAGCGCGACATCGGC 771

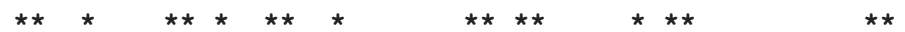

TCCAACCCTCGCGCCCTACGACGCCTCAGAACAGCAGCTGAACGGGCCAAGCGCACACTC 816 TCCAACCCTCGCGCCCTACGACGCCTCAGAACAGCAGCTGAACGGGCCAAGCGCACACTC 816 ATGAATCCTCGCGCACTGCGCCGCCTCCGCACAGCCGCCGAGCGCGCTAAGCGCACGCTG 816 CTGAACTCTCGCGCACTCCGACGCCTCCGCACGGCCGCTGAGCGCGCCAAGAGGACACTC 816 AACAACCCTCGAGCCATCAGACGACTGAGAACGGCTTGTGAACGAGCCAAGAGGACATTA 831 TCGAACAAGCGAGCGCTGCGCCGACTCCGCACTGCTTGCGAGAGGGCCAAGCGCACTCTG 822 TCGAACAAGCGAGCGCTGCGCCGACTCCGCACTGCTTGCGAGAGGGCCAAGCGCACTCTG 822 GGAAACAAACGTGCCATTCGAAGATTGAGAACGGCCTGTGAAAGAGCAAAGAGAACTTTG 819 TCGAATCCTCGTGCGCTTCGTCGTTTACGTACTGCATGCGAAAGAGCAAAACGAACATTG 822 TCAAACCCTCGTGCCCTTCGTCGCTTACGTACTGCATGCGAAAGAGCAAAAAGAACACTC 822 TCCAACCCACGTGCTCTTCGTCGTCTTCGTACCGCCTGCGAGCGCGCAAACGAGACTCTT 825 TCCAACCCACGTGCTCTTCGTCGTCTTCGTACCGCCTGCGAGCGCGCAAAGAGAACTCTT 825 GAAAACAAGCGTGCTCTACGTCGTTTACGTACTGCTTGTGAGCGTGCCAAGCGTACTCTT 822 GAAAACAAGCGTGCTCTACGTCGTTTACGTACTGCTTGTGAGCGCGCCAAGCGTACTCTT 822 GAAAACAAGCGTGCTGTCCGACGACTTAGAACTGCTTGTGAAAGGGCAAAGAGAACCCTT 825 GACAACAAGAGGGCTGTAAGACGTCTCCGAACAGCCTGTGAGCGTGCGAAGAGGACCCTG 819 GACAACAAGCGTGCTGTTCGTCGTCTGCGTACAGCATGTGAACGCGCGAAACGTACCTTG 822 GACAACAAGAGGGCTGTGAGACGTCTGAGAACCGCATGCGAGAGAGCAAAGAGGACACTT 822 GACAACAAGAGGGCTGTTCGCCGTCTCCGCACCGCATGTGAGAGGGCAAAGCGCACCCTG 822 GACAACAAGAGGGCGGTGCGTCGCCTGCGCACGGCCTGCGAGCGGGCCAAGCGCACGCTG 822 GAGAACAAGAGAGCTGTAAGACGCCTCCGTACTGCTTGTGAACGTGCTAAGCGTACCCTC 822 GAGAACAAGAGAGCTGTCAGGCGTCTCCGCACTGCCTGTGAGCGGGCCAAGCGCACCCTC 822 GAGAACAAGAGAGCAGTTCGCCGGCTACGCACAGCATGTGAACGTGCTAAGCGTACTCTC 822 CAGAACAAGCGGGCCGTGAGGCGGCTGCGCACCGCATGCGAGCGGGCCAAGAGAACCTTG 822 CAGAACAAGCGAGCCGTGAGGCGGCTGCGCACCGCCTGCGAGAGGGCCAAGAGGACCCTG 822 CAGAACAAGCGCGCGGTGCGGCGACTGCGCACGGCGTGCGAGAGGGCCAAGAGGACGCTG 822 CAGAACAAGAGAGCAGTGAGGAGACTGCGCACAGCTTGTGAGAGAGCAAAGAGGACCTTG 828 CAGAACAAGAGAGCCTTGAGGAGGCTGCGCACAGCTTGCGAGAGGGCCAAGAGGACCCTG 828 CAGAACAAGCGAGCCGTGAGGCGGCTGCGCACCGCCTGCGAGAGGGCCAAGAGGACCCTG 828 GGGAACAAGCGTGCCCTGCGCAGGCTGCGCACAGCCTGTGAGCGCGCCAAGCGCACCCTG 828 GGCAACAAGCGGGCGGTGCGGCGGCTGCGCACGGCGTGCGAGCGGGCCAAGCGCACGCTG 831

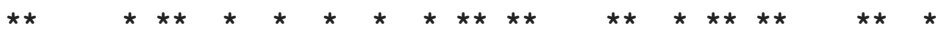

TCCTCTAGCACGGAGGCCACCATCGAGATCGACGCATTGTTTGAGGGCCAAGACTTCTAC 876 TCCTCCAGCACGGAGGCCACCATCGAGATTGACGCACTGTTTGAGGGCCAAGACTTCTAC 876 TCGTCCAGCCCCGAAGCCACCATCGAGATCGACGCGCTCTACGAGGGAATCGACTTCTAC 876 TCATCCAGCACCGAGGCCACCATCGAGATTGACGCTCTGTATGAGGGCATCGACTTCTAC 876 TCGACCAGTACACAATCGAATATAGAAATCGACTCTCTCTACGAAGGAATCGACTTCTAT 891 TCCTCGTCGGCCCAGGCCAACATCGAAATAGACTCGTTGCACGAGGGCGTCGACTTCTAC 882 TCCTCGTCGGCCCAGGCCAACATCGAAATAGACTCGTTGCACGAGGGCGTCGACTTCTAC 882 TCATCGTCTGCCCAGGCCAACATTGAGATTGACTCGTTGCATGAGGGAGTTGACTTTTAC 879 TCTAGTTCATCTCAAGCGAGTATCGAAATTGATTCGTTGTTCGAGGGGATCGATTTCTAC 882 TCTAGTTCGTCACAAGCTAGTATCGAAATTGATTCGCTATTCGAGGGCATTGATTTCTAC 882 TCGTCGTCTTGCCAGGCTTCGATTGAGATCGATTCTCTCTTCGAAGGAATTGACTTCTAC 885 TCGTCGTCTTCCCAGGCTTCAATTGAGATCGATTCTCTCTTCGAGGGAATTGACTTCTAC 885 
bioRxiv preprint doi: https://doi.org/10.1101/2020.09.21.307264; this version posted April 22, 2021. The copyright holder for this preprint (which was not certified by peer review) is the author/funder, who has granted bioRxiv a license to display the preprint in perpetuity. It is made available under aCC-BY-NC-ND 4.0 International license.

M nipponense KC460343

M_nipponense_DQ660140

Mytilus HSP70 AY861684

Perinereis_KŪ255783

Paralvinelīa_HSP70-2_EF580993

Haliotis_FJ812176

Oncorhynchus_HSC71_AAB21658

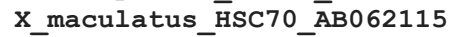

Human_HSPA8_HSC71_NP_006588

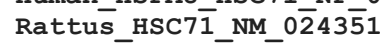

Pelodiscus_HS̄C70̄_HQ219723

BOS HSP70 Ū09861

Human_HSPĀ 1 A_NM_005345

Rattus HSP70 1A NM 031971

X_maculatus_H $\mathrm{H} S \mathrm{~T} \overline{7} 0-\overline{1}$ _AB0 62113

$x$ maculatus HSP70-2 AB062114

Hüman_HSPA1 $\overline{\mathrm{L}}$ NM $005 \overline{5} 27$

Human_HSPA6 $6 \overline{\mathrm{N}}$ P $\overline{0} 02146$

Alligātor_HS̄P70__AB306279

Drosophila_Bb_AF2 95957

Drosophila Aa AF295933

Spodoptera_FJ 754276

Bombyx_AB0 35326

Paralvinella_EF580992

B_plicatilis_HSP70-1_AB775784

B_plicatilis_HSP70-2_AB775785

A_brightwellì_HSP70_KX119429

Wūchereria_AF'167352

Setaria_AF-079360

C elegañs HSP70A M18540

C_elegans_HSP-1_NM_070667

M-nipponense_KC $460 \overline{3} 43$

M_nipponense_DQ660140

Mytilus HSP70 AY861684

Perinereis_KU 255783

Paralvinella_HSP70-2_EF580993

Haliotis FJ $8 \overline{1} 2176$

Oncorhynchus_HSC71_AAB21658

X_maculatus_HSC70_- $\mathrm{A} B 062115$

Human_HSPA8_HSC71_NP_006588

Rattus HSC7 $\overline{1}$ NM $0 \overline{2} 43 \overline{5} 1$

Pelodiscus_HS̄C70_HQ219723

Bos HSP70 Ü09861

Humān_HSPĀ 1 A_NM_005345

Rattus'HSP70_1A_NM_031971

X_maculatus_HSP70-1'AAB062113

X_maculatus_HSP70-2_AB062114

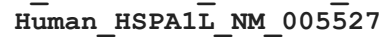

Human HSPA 6 NP $\overline{0} 02146$

Alligātor_HS̄P70_AB306279

Drosophila_Bb_AF2 95957

Drosophila_Aa_AF295933

Spodoptera_FJ754276

Bombyx_AB0 35326

Paralvinella_EF580992

B_plicatilis_HSP70-1_AB775784

B_plicatilis_HSP70-2_AB775785

A_brightwellī_HSP70_KX119429

Wüchereria_AF'167352

Setaria_AF-0 79360

C_elegañs_HSP70A_M18540

C_elegans_HSP-1_NM_070667

M-nipponense $\mathrm{KC} \overline{4} 60 \overline{3} 43$

M_nipponense_DQ660140

Mytilus_HSP70_AY861684

Perinerēis $\mathrm{KU} \overline{2} 55783$

Paralvinella_HSP70-2_EF580993

Haliotis_FJ812176

Oncorhynchus_HSC71_AAB21658

X_maculatus_ $\overline{\mathrm{H}} \mathrm{SC70}$ - $\mathrm{A} B 062115$

Human_HSPA8_HSC71_NP_006588

Rattus HSC7 $\overline{1}$ NM $0 \overline{2} 43 \overline{5} 1$

Pelodiscus_HS̄C70_HQ219723

Bos_HSP70_Ū09861

Humān_HSPĀ1A_NM_005345
TCAGCCTCTGCTCAAGCTAGTGTTGAGATTGATTCTCTGTATGAAGGTATTGATTTTTAC 882 TCAGCCTCTGCTCAAGCTAGTGTTGAGATTGATTCTCTGTATGAAGGTATTGATTTTTAC 882 TCTTCAAGCACACAAGCAAGTGTTGAGATTGACTCTCTGTTTGAAGGAGTTGACTTTTAT 885 TCCTCCAGCACCCAAGCCAGTATTGAAATTGATTCCTTGTATGAAGGTGTCGACTTCTAC 879 TCCAGCAGCACACAGGCAAGCATTGAAATAGATTCCCTCTATGAGGGCATAGACTATTAC 882 TCCTCCAGCACCCAGGCCAGCATAGAGATCGACTCCCTGTTTGAAGGAGTAGATTACTAC 882 TCCTCCAGCACCCAGGCCAGCATCGAGATCGACTCTTTGTACGAGGGAATCGACTTCTAC 882 TCTTCCAGCACGCAGGCCAGCATCGAGATCGACTCCCTGTACGAGGGCATCGACTTCTAC 882 TCTTCCAGCACCCAGGCCAGTATTGAGATCGATTCTCTCTATGAAGGAATCGACTTCTAT 882 TCCTCCAGCACCCAGGCCAGTATTGAGATTGATTCTCTCTATGAGGGAATTGACTTCTAC 882 TCTTCTAGCACTCAGGCCAGTATTGAAATTGACTCGCTCTATGAGGGCATTGATTTTTAC 882 TCGTCCAGCACCCAGGCCAGCCTGGAGATCGACTCCCTGTTCGAGGGCATCGACTTCTAC 882 TCGTCCAGCACCCAGGCCAGCCTGGAGATCGACTCCCTGTTTGAGGGCATCGACTTCTAC 882 TCGTCCAGCACCCAGGCCAGCCTGGAGATCGACTCTCTGTTCGAGGGCATCGACTTCTAC 882 TCCTCCAGCACTCAGGCAAGCATTGAGATTGACTCACTCTTTGAGGGCATTGACTTTTAC 888 TCCTCCAGCTCCCAGGCCAGCATCGAGATCGACTCTCTGTTCGAGGGTGTGGACCTGTAC 888 TCGTCCAGCACCCAGGCCAACCTAGAAATTGATTCACTTTATGAAGGCATTGACTTCTAT 888 TCCTCCAGCACCCAGGCCACCCTGGAGATAGACTCCCTGTTCGAGGGCGTGGACTTCTAC 888 AGCTCCTCCACGCAGGCCAGCATCGAGATCGACTCGCTGTTCGAGGGCATCGACTTCTAC 891

ACCAAAGTAAGCCGTGCCAGGTTTGAGGAGCTGTGCGCGGACCTCTTCCGCAACACCCTG 936 ACCAAAGTGAGCCGCGCCAGGTTTGAGGAGCTGTGCGCGGACCTCTTCCGCAACACCCTG 936 ACTCGCGTGTCCCGCGCGCGCTTCGAGGAGCTCAACGCTGACCTGTTCCGAGGCACCCTC 936 ACGCGAGTCTCCCGCGCCCGCTTCGAGGAACTGAACGCGGACCTGTTCAGGGGAACTCTG 936 ACAACTATTACCAGAGCCAGATTCGAAGAGCTCTGCTCCGATCTGTTCAGGTCAACTTTG 951 ACGAGCATCACCAGAGCTCGCTTTGAAGAGCTGTGCGCCGATTTGTTCCGCGGCACTCTC 942 ACGAGCATCACCAGAGCTCGCTTTGAAGAGCTGTGCGCCGATTTGTTCCGCGGCACCCTC 942 ACAAACATCACCAGAGCCAGATTCGAAGAGCTCAACGCTGATTTATTCCGCGGCTGTTTA 939 ACCAATATCACTCGTGCTCGCTTTGAGGAATTATGTGCTGATCTTTTCCGTTCAACTATG 942 ACTAATATTACTCGTGCTCGCTTTGAAGAACTTTGTGCTGATCTTTTCCGCTCAACAATG 942 ACCAACATCACTCGTGCTCGTTTCGAGGAGCTCTGCGCTGATCTCTTCAGATCCACCATG 945 ACCAACATCACTCGTGCTCGTTTCGAGGAGCTCTGCGCTGATCTCTTCAGATCCACCATG 945 ACTTCTATTACTCGTGCCAGGTTTGAGGAACTTTGTGGTGACTTGTTCCGTGGCACCCTT 942 ACTTCTATTACTCGTGCCAGGTTTGAGGAACTTTGTGGTGACTTGTTCCGTGGCACCCTG 942 ACAAGCATCACAAGAGCCAGGTTTGAGGAATTGAATGCAGATCTTTTCAGAGGAACCATG 945 ACAACCATCACCAGGGCTCGTTTTGAGGAGCTGTGTGCCGATCTCTTCCGAGGCACTCTC 939 ACAACAATCACTAGAGCTAGATTTGAGGAGCTGAATGCTGACCTATTCCGTGGTACGCTG 942 ACCAGCATCACTAGAGCCAGGTTTGAGGAGCTCAATGCTGATCTTTTCCGTGGCACACTA 942 ACCTCCATCACCAGGGCTCGCTTTGAGGAGCTCAATGCAGACCTTTTCCGTGGCACCCTT 942 ACGTCCATCACCAGGGCGCGCTTTGAGGAGCTGAACGCGGACCTGTTCCGCGGAACGCTG 942 ACCTCCATTACCCGTGCCCGATTTGAAGAACTGAATGCTGACCTGTTCCGTGGCACCCTG 942 ACCTCCATTACCCGTGCTCGATTTGAGGAGTTGAATGCTGACCTGTTCCGTGGCACACTG 942 ACCTCAATTACCAGAGCTCGTTTTGAAGAGTTAAATGCTGATCTGTTCCGTGGCACTCTG 942 ACGTCCATCACCAGGGCGCGGTTCGAGGAGCTGTGCTCCGACCTGTTCCGGAGCACCCTA 942 ACGTCCATCACCAGGGCGAGGTTCGAGGAGCTGTGCTCCGACCTGTTCCGAAGCACCCTG 942 ACGTCCATCACGCGGGCGCGGTTCGAGGAGCTGTGCTCGGACCTGTTCCGCGGCACGCTG 942 ACCTCAATCACAAGGGCACGCTTCGAGGAGCTCAACTCAGACCTCTTTAGGGGAACACTG 948 ACCTCCATCACCAGGGCTCGCTTTGAGGAGCTGTGCTCCGACCTGTTCAGGGGAACCTTG 948 ACATCCATCACCAGAGCTCGATTTGAAGAGTTGTGTGCAGACCTGTTTAGGGGTACCCTG 948 ACGTCCATCACTCGTGCCCGCTTTGAGGAACTGTGCTCAGACCTCTTCCGCAGCACCCTG 948 ACGTCCATCACGCGGGCGCGCTTCGAGGAGCTGAACGCCGACCTGTTCCGCGGCACGCTG 951

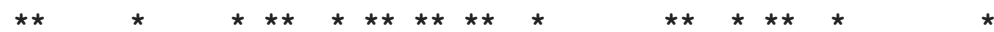

CAGCCTGTGGAGAAGGCCCTCAACGATGCCAAGATGGACAAGGGTCAGATCCACGACATC 996 CAGCCTGTGGAGAAGGCCCTCAACGATGCCAAGATGGATAAGGGTCAGATCCACGACATC 996 GAGCCGGTCGAGAAAGCGCTCAAGGACGCCAAGATGGACAAGAGCCAGATCCACGACGTC 996 GAACCCGTCGAGAAGGCACTCAAGGATGCTAAACTCGACAAGAGTCAGATCCACGACGTG 996 GAGCCAGTAGAGAAAGCTCTCCGAGATGCCAAGATGGACAAGTCCAAGATCGACGAAGTG 1011 GACCCAGTGGAGAAGGCGCTGCGAGACGCCAAAATGGACAAGTCAAGTGTGAACGAGATT 1002 GACCCAGTGGAGAAGGCGCTGCGAGACGCCAAAATGGACAAGTCAAGTGTGAACGAGATT 1002 GACCCAGTCGAGAAATCTTTGAGAGACGCTAAACTCGACAAAGCCCAGATCCATGAAATT 999 GATCCAGTTGAGAAAGCACTCCGTGATGCTAAGATGGACAAGGCCCAAGTCCATGACATT 1002 GATCCAGTTGAGAAAGCATTGCGTGATGCCAAGATGGATAAGGCACAAGTTCATGATATT 1002 GACCCAGTCGAGAAGTCTCTCCGTGACGCCAAGATGGACAAGAGCCAAGTTCATGACATC 1005 GACCCAGTCGAGAAGTCTCTCCGTGACGCCAAGATGGACAAGAGCCAAGTTCATGACATC 1005 GAGCCAGTTGAGAAGTCTCTGAGAGATGCAAAAATGGACAAAGCCCAAATCTATGACATT 1002 GAGCCAGTTGAGAAGTCTCTGAGAGATGCAAAAATGGACAAAGCCCAAATCCATGACATT 1002 GAACCAGTTGAAAAAGCTCTACGCGATGCCAAACTAGACAAGGCTGCTGTCCATGAAATT 1005 GAGCCAGTAGAGAAGTCCATCCGTGATGCCAAGATGGGAAAAGATGCCATCCACGACATC 999 GAACCTGTAGAAAAGTCTCTGCGTGATGCCAAGCTGGACAAGTCCAGCATCCATGACATC 1002 GAGCCAGTGGAGAAGGCTTTACGTGATGCCAAGGCAGACAAGGTCTCCATCCATGACATA 1002 GACCCAGTGGAGAAATCCCTCCGCGACGCCAAGATGGACAAAGCCCAGGTACACGACATC 1002 GAGCCCGTGGAGAAGTCTCTCCGAGACGCCAAGATGGACAAGGCTCAGATCCACGAGATT 1002 GACCCAGTAGAGAAAGCCCTTCGAGATGCCAAACTAGACAAGTCACAGATTCATGATATT 1002 GACCCTGTAGAGAAGGCCCTTCGAGATGCCAAACTAGACAAGTCACAGATCCATGATATT 1002 GATCCTGTGGAGAAGGCCCTGCGGGATGCCAAACTAGACAAATCACAGATCCATGACATT 1002 GAGCCCGTGGAGAAGGCGCTACGCGACGCCAAGCTGGACAAGGCGCAGATCCACGACCTG 1002 GAGCCCGTGGAGAAGGCTCTGCGCGACGCCAAGCTGGACAAGGCCCAGATTCACGACCTG 1002 
bioRxiv preprint doi: https://doi.org/10.1101/2020.09.21.307264; this version posted April 22, 2021. The copyright holder for this preprint (which was not certified by peer review) is the author/funder, who has granted bioRxiv a license to display the preprint in perpetuity. It is made available under aCC-BY-NC-ND 4.0 International license.

Rattus HSP70 1A NM 031971

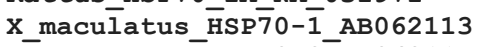
$x$ maculatus HSP70-2 AB0 62114 Human_HSPA1 $\overline{\mathrm{L}}$ NM $005 \overline{5} 27$ Human HSPA $6 \overline{\mathrm{N} P} \overline{0} 02146$ Alligätor_HSPPTO_AB306279

Drosophila_Bb_AF295957 Drosophila Aa AF295933 Spodoptera_FJ754276 Bombyx $\mathrm{AB} 0 \overline{3} 5326$

Paralvinella_EF580992 B plicatilis HSP70-1 AB775784 B_plicatilis_HSP70-2_AB775785 A brightwelli HSP70 KX119429 Wüchereria AF'167352 Setaria_AF079360 C elegañs HSP70A M18540 C_elegans_HSP-1_NM_070667 Mnipponense_KC $\overline{4} 60 \overline{3} 43$ M_nipponense_DQ660140 Mytilus HSP70 AY861684 Perineréis KU $\overline{2} 55783$ Paralvinelía HSP70-2 EF580993 Haliotis_FJ812176 Oncorhynchus_HSC71_AAB21658 X_maculatus_HSC70_ABD062115 Hüman_HSPA8_HSC71_NP_006588 Rattus_HSC7 $\overline{1}$ NM $0 \overline{2} 43 \overline{5} \overline{1}$ Pelodiscus_HS̄C70̄HQ219723 Bos HSP70 Ū09861 Humān_HSPĀ1A_NM_005345 Rattus HSP70 1A NM 031971

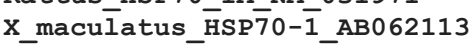
$X$ maculatus HSP70-2 AB062114 Human_HSPA1]_NM_0055 27 Human HSPA 6 NP $\overline{0} 02146$ Alligātor_HS̄P7 $\overline{0}$ _AB306279

Drosophila_Bb_AF2 95957 Drosophila Aa AF295933 Spodoptera_FJ754276 Bombyx $\mathrm{AB} 0 \overline{3} 5326$

Paralvinella_EF580992 B_plicatilis_HSP70-1_AB775784 B plicatilis_HSP70-2 AB775785 A_brightwellì_HSP70_KX119429 Wüchereria AF' 67352 Setaria_AF-79360 C elegans HSP70A M18540 C_elegans_HSP-1_NM_070667 Mnipponense_KC $\overline{4} 60 \overline{3} 43$ M_nipponense_DQ660140 Mytilus HSP70 AY861684 Perinerēis KU $\overline{2} 55783$ Paralvinelīa_HSP70-2_EF580993 Haliotis_FJ812176 Oncorhynchus_HSC71_AAB21658 $X$ maculatus_H $\mathrm{H} C 70$ A $\mathrm{A} 062115$ Human HSPA8 HSC71-NP 006588 Rattus_HSC7 $\overline{1}$ NM $0 \overline{2} 43 \overline{5} 1$ Pelodiscus_HSC7 $\overline{0}$ HQ219723 Bos HSP70 Ü09861 Humān_HSPĀ 1 A_NM_005345 Rattus_HSP70_1A_NM_031971 $X$ maculatus_ $\bar{H} S P \overline{7} 0-\overline{1}$ AB0 62113 $X$ maculatus_HSP70-2_AB062114 Hüman HSPA1 $\overline{\mathrm{L}}$ NM $005 \overline{5} 27$ Human HSPA $6 \overline{\mathrm{N} P} \overline{0} 02146$ Alligätor_HS̄P70_AB306279

Drosophila_Bb_AF295957 Drosophila_Aa-AF295933 Spodoptera_FJ754276 Bombyx_AB0 $\overline{3} 5326$ Paralvinella_EF580992
GAGCCCGTGGAGAAGGCCCTGCGCGACGCCAAGCTGGACAAGGCGCAGATCCACGACCTG 1002 GAGCCAGTTGAGAAGGCCCTACAAGATGCTAAGCTGGATAAATCCAAGATCCACGAAGTT 1008 GAGCCTGTGGAGAAGGCTTTGAGGGACGCCAAGATGGACAAGGGCCAGATCCATGACGCG 1008 GAGCCTGTAGAAAAAGCGCTTCGGGATGCCAAGATGGATAAGGCTAAAATCCATGACATT 1008 GAGCCGGTGGAGAAGGCCCTGCGGGATGCCAAGCTGGACAAGGCCCAGATTCATGACGTC 1008 GAGCCCGTGGAGAAGGCGCTGCGCGACGCCAAGCTGGACAAGGGGCAGGTGCAGGAGATC 1011

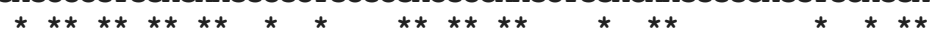

GTGCTCGTCGGCGGATCCACTCGCATTCCCAAGGTGCAAAGTCTGCTGCAGGAGTTCTTC 1056 GTGCTCGTCGGCGGATCCACTCGCATTCCCAAGGTGCAAAGTCTGCTGCAGGACTTCTTC 1056 GTTCTCGTCGGAGGCTCGACCAGAATCCCGAAGGTGCAGAGTCTGCTGCAAAACTTCTTC 1056 GTCCTCGTCGGAGGCTCCACCCGCATTCCGAAAGTACAAACTATGCTCCAAAACTTCTTC 1056 GTTCTGGTCGGAGGATCGACTAGAATCCCGAAGATCCAGAAACTGCTACAGGAGTTCATG 1071 GTGTTGGTGGGCGGCTCGACCCGCATTCCAAAAGTTCAAAAGCTGCTCCAGGACTTTTTC 1062 GTGTTGGTGGGCGGCTCGACCCGCATTCCAAAAGTTCAAAAGCTGCTCCAGGACTTTTTC 1062 GTTTTAGTCGGTGGATCGACCAGAATCCCGAAAGTGCAAAAATTATTACAAGACTTTTTC 1059 GTGCTAGTAGGTGGATCAACCCGTATCCCGAAAGTGCAGAAGCTCTTGTCAGATTTCTTT 1062 GTACTGGTGGGTGGGTCAACTCGTATCCCGAAAGTGCAGAAACTGCTCTCGGATTTCTTC 1062 GTCCTTGTCGGAGGATCCACTCGTATCCCAAAGGTCCAGAAACTTTTGTCCGATCTCTTC 1065 GTCCTTGTCGGAGGATCCACTCGTATCCCAAAGGTCCAGAAACTTTTGTCCGATCTCTTC 1065 GTCCTTGTTGGAGGTTCCACCAGAATTCCAAAGATTCAGAAGCTGTTACAGGATTTCTTC 1062 GTCCTTGTTGGAGGTTCCACCAGAATTCCAAAGATTCAGAAGCTGTTACAGGATTTCTTC 1062 GTCTTGGTAGGTGGATCAACCAGAATTCCAAAAATCCAGAAGTTACTTCAGGACTTTTTT 1065 GTGTTGGTCGGTGGTTCCACCCGTATCCCCAAGATCCAGAAACTCCTTCAGGATTTCTTC 1059 GTACTGGTTGGTGGCTCCACCAGAATTCCCAAGATCCAAAAACTGCTGCAGGACTTTTTC 1062 GTGCTGGTTGGGGGATCTACACGTATCCCCAAGATCCAGAAACTCCTCCAAGACTTCTTC 1062 GTCCTGGTCGGAGGCTCCACTCGTATCCCCAAGATCCAGAAACTGCTCCAAGATTTCTTC 1062 GTCTTGGTGGGAGGCTCGACCCGCATCCCAAAGATCCAAAAGCTCCTGCAGGACTTCTTT 1062 GTCCTGGTTGGTGGTTCTACTCGTATCCCCAAGATTCAGAAGCTTCTCCAAGACTTCTTC 1062 GTCCTGGTGGGTGGTTCTACCAGAATCCCCAAGATCCAGAAACTTCTGCAAGACTTCTTC 1062 GTACTGGTTGGTGGATCAACCCGTATTCCCAAGATTCAGAAACTCCTGCAGGATTTCTTC 1062 GTCCTGGTGGGGGGCTCCACCCGCATCCCCAAGGTGCAGAAGCTGCTGCAGGACTTCTTC 1062 GTCCTGGTCGGGGGCTCCACCCGCATCCCCAAGGTGCAGAAGCTGCTGCAGGACTTCTTC 1062 GTGCTGGTGGGCGGCTCGACGCGCATCCCCAAGGTGCAGAAGCTGCTGCAGGACTTCTTC 1062 GTCCTAGTTGGTGGCTCTACAAGAATCCCCAAAATCCAAAAGCTCCTGCAGGACTTCTTC 1068 GTCCTGGTGGGAGGCTCCACCCGAATCCCCAAGATCCAGAAGCTGCTGCAGGACTTCTTC 1068 GTTTTAGTAGGGGGCTCCACCCGCATCCCCAAGGTGCAGCGGCTGCTTCAGGACTACTTC 1068 GTCCTGGTGGGGGGCTCCACACGCATCCCCAAGGTGCAGAAGTTGCTGCAGGACTTCTTC 1068 GTGCTGGTGGGCGGCTCGACGCGCATCCCCAAGATCCAGAAGCTGCTGCAGGACTTCTTC 1071

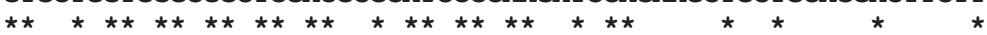

CACGGCAAGAACCTCAACCTATCCATCAACCCAGACGAGGCAGTGGCATACGGAGCTGCT 1116 CACGGCAAGAACCTCAACCTATCCATCAACCCAGACGAGGCAGTGGCATACGGAGCTGCT 1116 TGCGGCAAGAAGCTGAATTTGTCCATCAACCCGGACGAGGCGGTCGCGTATGGCGCGGCC 1116 TGTGGGAAGAAACTGAACTTATCCATCAATCCGGACGAAGCCGTAGCCTACGGTGCAGCA 1116 AACGGGAAAGAGCTGAACAAGTCGATCAATCCCGATGAAGCAGTGGCCTATGGAGCGGCC 1131 AATGGCAAAGAGCTGAACAAGTCGATTAACCCGGACGAGGCGGTGGCGTACGGCGCGGCC 1122 AATGGCAAAGAGCTGAACAAGTCGATTAACCCGGACGAGGCGGTGGCGTACGGCGCGGCC 1122 AATGGAAAAGAGTTGAACAAGAGCATTAACCCAGACGAGGCCGTGGCCTACGGTGCTGCC 1119 TCTGGCAAAGAATTGAATAAAAGCATCAATCCGGACGAGGCCGTGGCGTATGGTGCAGCA 1122 TCTGGCAAAGAATTGAACAAAAGTATCAACCCTGATGAGGCTGTGGCATATGGTGCTGCT 1122 TCAGGAAAGGAATTGAACAAGTCCATCAACCCAGATGAGGCGTTAGCCTACGGAGCTGCC 1125 TCAGGAAAGGAATTGAACAAGTCCATCAACCCAGATGAGGCCGTTGCCTACGGAGCTGCC 1125 AATGGAAAGGAGCTCAACAAATCCATCAATCCTGATGAAGCTGTAGCTTATGGTGCAGCT 1122 AATGGAAAGGAGCTCAACAAATCCATCAATCCTGATGAAGCTGTAGCTTATGGTGCAGCT 1122 CAAGGCAAAGAATTGAACAAATCCATTAACCCTGATGAAGCTGTAGCATACGGTGCAGCT 1125 AACGGCAAAGAATTGAACAAATCTATCAACCCTGATGAGGCTGTCGCATACGGAGCTGCC 1119 AACGGCAAAGAACTAAACAAGTCGATCAACCCTGATGAAGCTGTTGCATATGGTGCAGCT 1122 AACGGCAAGGAGCTATGCAAGAGCATCAACCCTGATGAGGCTGTTGCTTATGGTGCTGCC 1122 AACGGCAAAGAGCTCAACAAAAGCATCAACCCCGACGAAGCTGTGGCCTATGGCGCAGCT 1122 AACGGGAAGGAACTCAACAAGAGCATTAACCCTGACGAGGCGGTGGCGTACGGTGCAGCT 1122 AATGGAAAAGAACTGAATAAGAGCATCAACCCTGATGAAGCTGTTGCTTATGGTGCAGCT 1122 AATGGAAAAGAGCTGAATAAGAGCATTAACCCCGATGAAGCTGTTGCCTATGGTGCAGCT 1122 AATGGGAAAGAGCTGAACAAGAGCATCAATCCTGATGAAGCTGTGGCTTACGGTGCAGCT 1122 AACGGGCGCGACCTCAACAAGAGCATCAACCCCGACGAGGCGGTGGCGTACGGGGCGGCG 1122 AACGGGCGCGACCTGAACAAGAGCATCAACCCCGACGAGGCTGTGGCCTACGGGGCGGCG 1122 AACGGGCGCGACCTGAACAAGAGCATCAATCCGGACGAGGCGGTGGCCTACGGGGCGGCG 1122 AATGGTAGAGAACTAAACAAGAGCATCAACCCTGATGAAGCGGTGGCCTATGGTGCAGCA 1128 AACGGCCGGGAGCTGAACAAGAGCATCAACCCGGACGAGGCGGTGGCTTACGGCGCCGCC 1128 AATGGACGTGATCTCAACAAGAGCATCAACCCTGATGAGGCCGTAGCATATGGGGCTGCG 1128 AACGGCAAGGAGCTGAACAAGAGCATCAACCCTGATGAGGCTGTGGCCTATGGGGCTGCT 1128 AATGGCAAGGAGCTGAACAAGAGCATCAACCCCGACGAGGCGGTGGCGTACGGCGCGGCG 1131

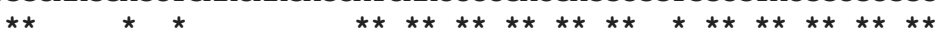

GTGCAGGCCGCTATCCTCAGCGGAGACCAGAGCGGCAAGATCCAGGACGTGCTGCTGGTG 1176 GTGCAGGCCGCTATCCTCAGCGGAGACCAGAGCGGCAAGATCCAGGACGTGCTGCTGGTG 1176 GTCCAGGCGGCCATCCTGAGTGGCGAACAAGACTCGAAGATCCAGGACGTGTTGCTGGTG 1176 GTCCAGGCAGCCATCCTGAGCGGCGAGACCGACTCCAAGATACAGGACGTCTTGTTGGTC 1176 GTTCAAGCAGCTATCCTGACAGGTTCTGAACACTCGGCCGTTAAAGATCTACTCTTGGTA 1191 
bioRxiv preprint doi: https://doi.org/10.1101/2020.09.21.307264; this version posted April 22, 2021. The copyright holder for this preprint (which was not certified by peer review) is the author/funder, who has granted bioRxiv a license to display the preprint in perpetuity. It is made available under aCC-BY-NC-ND 4.0 International license.

B plicatilis HSP70-1 AB775784 B_plicatilis_HSP70-2_AB775785 A brightwellì HSP7O $\bar{K} X 119429$ Wüchereria_AF'167352 Setaria AF 079360 C_elegañs_HSP70A_M18540 C_elegans_HSP-1_NM_070667 M nipponense KC $\overline{4} 60 \overline{3} 43$ M_nipponense-DQ660140 Mýtilus_HSP70_AY861684 Perinerēis_KU 255783 Paralvinelīa HSP70-2 EF580993 Haliotis_FJ81̄2176 Oncorhynchus HSC71 AAB21658

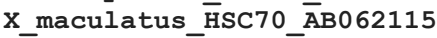
Human_HSPA8_HSC71_NP_006588 Rattus_HSC7 $\overline{1}$ NM $0 \overline{2} 43 \overline{5} \overline{1}$ Pelodiscus_HS̄C70_HQ219723 Bos_HSP70_Ū09861 Humān_HSPĀ1A_NM_005345 Rattus_HSP70_1A_NM_031971

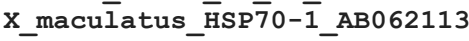
$x$ maculatus HSP70-2 AB062114 Human_HSPA1 $\overline{\mathrm{L}}$ NM $005 \overline{5} 27$ Human HSPA $6 \overline{\mathrm{N}} \mathrm{P}-\overline{0} 02146$ Alligātor_HS̄P70_AB306279

Drosophila_Bb_AF295957 Drosophila_Aa_AF295933 Spodoptera_FJ754276 Bombyx AB0 $\overline{3} 5326$ Paralvinella_EF580992 B plicatilis HSP70-1 AB775784 B_plicatilis HSP70-2 AB775785 A brightwelli HSP70 KX119429 Wüchereria AF'167352 Setaria AF 079360 C_elegañs_HSP70A_M18540 C_elegans_HSP-1_NM_070667 Mnipponense KC $\overline{4} 60 \overline{3} 43$ M_nipponense_DQ660140 Mytilus_HSP70 AY861684 Perinerēis KU $\overline{2} 55783$ Paralvinelía HSP70-2 EF580993 Haliotis_FJ81̄2176 Oncorhynchus_HSC71_AAB21658 X_maculatus_HSC70_AB062115 Human_HSPA8 HSC71_NP_006588

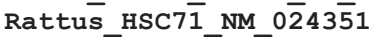
Pelodiscus_HS̄C70̄HQ219723 Bos HSP70 Ū09861 Humān_HSPĀ1A_NM_005345 Rattus HSP70-1A-NM 031971 X_maculatus_HSP70-1. $x$ maculatus HSP70-2 AB062114 Human_HSPA1 $\overline{\mathrm{L}}$ NM $005 \overline{5} 27$ Human HSPA $6 \overline{\mathrm{N} P} \overline{0} 02146$ Alligātor_HS̄P7 $\overline{0}$ _AB306279

Drosophila_Bb_AF295957 Drosophila Aa AF295933 Spodoptera_FJ754276 Bombyx AB0 $\overline{3} 5326$

Paralvinella_EF580992 B_plicatilis_HSP70-1_AB775784 B_plicatilis_HSP70-2 AB775785 A_brightwellíi_HSP70_KX119429 Wüchereria AF'167352 Setaria_AF 079360 C elegans HSP70A M18540 C_elegans_HSP-1_NM_070667 Mnipponense KC $\overline{4} 60 \overline{3} 43$ M_nipponense_DQ660140 Mytilus HSP70 AY861684 Perinerēis KU $\overline{2} 55783$ Paralvinelīa_HSP70-2_EF580993 Haliotis_FJ812176
GTCCAGGCGGCCATTTTGACCGGCGACAAGTCCGAGGCCGTCCAGGACCTGCTGCTGCTT 1182 GTCCAGGCGGCCATTTTGACCGGCGACAAGTCCGAGGCCGTCCAGGACCTGCTGCTGCTT 1182 GTCCAAGCCGCTATTTTAACGGGTGATAAATCCGAAGCCGTTCAAGATTTATTGTTATTA 1179 GTGCAGGCAGCTATTCTTTCTGGTGATAAGTCGGAAGCCGTACAAGATTTGTTACTGCTT 1182 GTCCAGGCAGCTATCCTCTCTGGTGATAAGTCAGAAGCCGTACAAGATTTGTTATTCGTC 1182 GTCCAAGCCGCTATCCTCTCCGGAGACAAGTCTGAGGCTGTCCAGGATCTTCTTCTTCTT 1185 GTCCAAGCCGCTATCCTCTCCGGAGACAAGTCTGAGGCTGTCCAGGATCTTCTTCTTCTT 1185 GTACAGGCAGCCATATTGTGTGGTGACAAGTCTGAAGCAGTTCAAGATTTGTTGCTTTTG 1182 GTACAGGCAGCCATATTGTGTGGTGACAAGTCTGAAGCAGTTCAAGATTTGTTGCTTTTG 1182 GTGCAGGCAGCCATTTTGTCAGGTGACAAGTCAGAAGAGGTACAAGACTTGTTATTGTTA 1185 GTTCAGGCTGCCATCTTGCACGGTGACAAGTCAGAAGAGGTCCAAGACTTGCTTTTGCTT 1179 GTCCAGGCTGCCATCCTTCATGGAGACAAGTCCGAGGCAGTTCAGGATCTGCTGCTACTG 1182 GTCCAGGCTGCTATCCTCCATGGTGACAAGTCCGAGGAAGTCCAAGACCTGCTACTGCTG 1182 GTCCAGGCAGCCATACTCTCAGGTGACAAGTCTGAGAATGTCCAGGACCTGCTTTTGCTG 1182 GTCCAGGCGGCCATCTTGTGCGGAGACAAATCCGAGATTGTTCAGGACCTGCTTCTGTTG 1182 GTCCAGGCAGCCATCTTGTCTGGAGACAAGTCTGAGAATGTTCAAGATTTGCTGCTCTTG 1182 GTCCAGGCAGCCATTCTATCTGGAGACAAGTCTGAGAATGTTCAGGATTTGCTGCTCTTG 1182 GTTCAGGCAGCAATCTTGTCTGGGGACAAGTCTGAGAATGTACAAGACCTGCTACTGTTG 1182 GTGCAGGCGGCCATCCTGATGGGGGACAAGTCGGAGAACGTGCAGGACCTGCTGTTGCTG 1182 GTGCAGGCGGCCATCCTGATGGGGGACAAGTCCGAGAACGTGCAGGACCTGCTGCTGCTG 1182 GTGCAGGCGGCCATCCTGATGGGGGACAAGTCGGAGAACGTGCAGGACCTGCTGCTGCTG 1182 GTCCAGGCTGCTATCCTCATGGGTGACACCTCAGAAAATGTCCAAGATTTACTGCTCCTG 1188 GTCCAGGCCGCCATCCTGACGGGAGACACGTCGGGGAACGTCCAGGACCTGCTGCTGCTG 1188 GTACAAGCAGCCATCCTGATGGGGGACAAGTCTGAGAAGGTACAGGACCTGCTGCTGCTG 1188 GTGCAGGCGGCCGTGTTGATGGGGGACAAATGTGAGAAAGTGCAGGATCTCCTGCTGCTG 1188 GTGCAGGCCGCCATCCTCATGGGCGACAAGTCGGAGAACGTGCAGGACCTGCTGCTGCTG 1191 $\star * \star * * * * * * * * * * * * * * *$

GACGTGGCCCCACTTTCATTGGGAATTGAGACCGCTGGAGGTGTAATGACCAAGCTGATC 1236 GACGTGGCCCCACTTTCATTGGGAATTGAGACCGCTGGAGGTGTAATGACCAAGCTGATC 1236 GACGTGGCCCCTCTGTCCCTCGGCATCGAAACCGCCGGCGGCGTGATGACGAAGATCATC 1236 GACGTGGCTCCTCTGTCCCTCGGTATTGAGACAGCCGGCGGCGTGATGACGAAGATCATC 1236 GACGTGACGCCATTATCTTTAGGTCTGGAGACGGCTGGAGGCGTGATGACGAAACTGGTG 1251 GACGTGGCGCCTCTATCGCTGGGCATTGAGACGGCCGGCGGCGTCATGACCGCCCTCATC 1242 GACGTGGCGCCTCTATCGCTGGGCATTGAGACCGCCGGCGGCGTCATGACCGCCCTCATC 1242 GACGTTGCTCCTTTGTCTCTTGGTATCGAGACTGCCGGCGGTGTTATGACATCATTGATC 1239 GACGTCGCACCGCTTTCACTTGGTATTGTAACAGCTGGGGGTGTTATGACTGCGCTTATC 1242 GACGTCGCACCGCTTTCACTTGGTATCGAGACTGCTGGTGGTGTTATGACTGCTCTCATT 1242 GACGTTGCCCCACTTTCCCTTGGTATTGAGACCGCTGGAGGAGTCATGACTGCTCTCATC 1245 GACGTTGCCCCACTTTCCCTTGGTATTGAGACCGCTGGAGGAGTCATGACTGCTCTCATC 1245 GATGTAACACCACTGTCTCTTGGTATTGAAACTGCTGGTGGTGTCATGACTGCCCTTATA 1242 GATGTAACACCTTTGTCTCTTGGTATTGAAACTGCTGGAGGTGTTATGACAGCACTTATT 1242 GATGTTACCCCACTGTCTCTTGGTATTGAAACAGCTGGAGGTGTGATGACAGCTCTAATC 1245 GATGTGACCCCCTTGTCCCTTGGTATCGAGACCGCGGGTGGCGTCATGACCTCCTTGATC 1239 GATGTGACACCTCTGTCTCTGGGTATAGAGACAGCCGGTGGTGTGATGACGTCGCTGATC 1242 GATGTCACTCCACTGTCACTTGGTATCGAGACTGCTGGCGGTGTGATGACTGTCCTCATC 1242 GACGTCACACCCCTCTCCCTGGGTATTGAGACCGCTGGAGGTGTCATGACCGTCCTGATC 1242 GACGTCACGCCCTTGTCTCTGGGCATCGAGACGGCAGGCGGAGTCATGACCGTGCTGATC 1242 GATGTCACTCCTCTTTCCCTTGGTATTGAAACTGCTGGTGGAGTCATGACTGTCCTCATC 1242 GATGTCACTCCTCTTTCCCTTGGGATTGAAACTGCTGGTGGAGTCATGACTGTCCTCATC 1242 GATGTCACTCCTCTGTCTCTGGGTATTGAAACAGCAGGTGGTGTCATGACAGTCCTGATC 1242 GACGTGGCTCCCCTGTCGCTGGGACTGGAGACGGCCGGAGGCGTGATGACCGCCCTGATC 1242 GACGTGGCTCCCCTGTCGCTGGGGCTGGAGACGGCCGGAGGCGTGATGACTGCCCTGATC 1242 GACGTGGCGCCGCTGTCGCTGGGTCTGGAGACCGCGGGCGGCGTGATGACGGCGCTCATC 1242 GATGTGGCACCCTTGTCGCTGGGCATTGAGACAGCCGGTGGCGTTATGACACCTCTTATC 1248 GACGTGGCTCCTCTGTCCCTGGGGATCGAGACGGCCGGAGGAGTGATGACTGCCCTGATC 1248 GACGTGGCTCCCCTGTCCCTGGGGCTGGAGACGGCTGGGGGCGTGATGACTGCCCTGATA 1248 GATGTGGCTCCCCTGTCTCTGGGGCTGGAGACAGCAGGTGGGGTGATGACCACGCTGATC 1248 GACGTGACGCCGCTGTCGCTCGGCATCGAGACGGCGGGCGGCGTCATGACGGCGCTCATC 1251

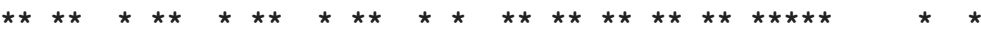

GAGCGCAACTGTCGCATTCCGTGCAAGCAGACTAAGACGTTCTCCACGTACTCGGACAAC 1296 GAGCGCAACTGCCGCATTCCGTGCAAGCAGACTAAGACATTCTCCACATACGCGGACAAC 1296 GAGCGCAACTGTAAGATCCCGTGCAAGCAGTCGCAGACGTTCACCACGTACTCGGACAAC 1296 GAACGAAACTCAAAGATTCCGTGCAAACAGTCTCAGACATTCACGACGTACTCAGACAAC 1296 GAACGTAACACGACCATTCCTCACAAGACGTCTAAGACATTCACCACCTACTCTGATAAT 1311 AAACGCAACACCACCATACCCACCAAGCAGACACAGACGTTCACCACTTATGCGGACAAC 1302 AAACGCAACACCACCATACCCACCAAGCAGACACAGACGTTCACCACTTATGCGGACAAC 1302 AAGAGAAACACCACAATCCCGACTAAACAAACCCAAGTTTTCACTACTTATTCAGACAAC 1299 AAGCGAAATACCACTATTCCCACGAAAACCTCCCAAACTTTTACAACATACTCCGACAAC 1302 AAGCGAAACACTACCATCCCGACGAAAACGTCGCAAACCTTTACCACCTACTCTGATAAT 1302 AAGAGAAACACCACCATCCCAACCAAGACCGCTCAGACCTTCACAACCTATTCTGATAAC 1305 AAGAGAAACACCACCATCCCAACCAAGACCGCTCAGACCTTCACAACCTATTCTGATAAC 1305 AAAAGAAACACCACCATTCCAACCAAACAGACCCAGACCTTTACCACCTATTCTGACAAC 1302 AAGCGTAACACAACAATCCCAACCAAGCAGACTCAGACATTCACCACTTATTCAGACAAT 1302 AAACGTAATACAACCATTCCAACAAAACAGACACAAACCTTCACTACCTACTCTGACAAT 1305 AAGAGGAACACAACTATCCCCACCAAGCAGACACAGACCTTCACAACCTATTCCGACAAC 1299 AAGCGCAACACGACCATTCCAACAAAACAGACCCAAACCTTCACCACCTACTCGGACAAT 1302 AAGAGGAACACAACCATCCCCACCAAACAGACCCAGACCTTCACAACATACTCAGACAAT 1302 
bioRxiv preprint doi: https://doi.org/10.1101/2020.09.21.307264; this version posted April 22, 2021. The copyright holder for this preprint (which was not certified by peer review) is the author/funder, who has granted bioRxiv a license to display the preprint in perpetuity. It is made available under aCC-BY-NC-ND 4.0 International license.

Oncorhynchus HSC71 AAB21658 $\mathrm{X}$ maculatus $\overline{\mathrm{H} S C 70} \overline{\mathrm{A}} \mathrm{B} 062115$ Human HSPA8 ${ }_{\text {HSC71 NP_006588 }}$

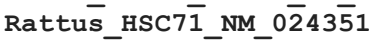
Pelodiscus HS $\mathrm{C} 7 \overline{0}$ HQ219723 Bos_HSP70_Ū09861 Humān_HSPĀ1A_NM_005345 Rattus HSP70_1A-NM 031971

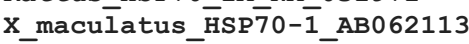
X_maculatus_HSP70-2_AB062114 Human_HSPA1 $\overline{\mathrm{L}}$ NM $005 \overline{5} 27$ Human HSPA $6 \overline{\mathrm{N}} \mathrm{P} \overline{0} 02146$ Alligator_HSP7 $\overline{0}$ _AB306279

Drosophila Bb AF295957 Drosophila_Aa_AF2 95933 Spodoptera FJ754276 Bombyx_AB0 35326 Paralvinella_EF580992 B_plicatilis_HSP70-1_AB775784 B_plicatilis_HSP70-2_AB775785 A brightwelli HSP70 $\overline{\mathrm{K}} \times 119429$ Wüchereria $\mathrm{AF} \overline{1} 67352$ Setaria $\mathrm{AF} \overline{0} 79360$ C_elegañs_HSP70A_M18540 C-elegans_HSP-1_NM_070667 M_nipponeñse_kC $\overline{4} 60 \overline{3} 43$ M_nipponense-DQ660140 Mȳtilus_HSP70 AY 861684 Perinerēis_KŪ255783 Paralvinelía_HSP70-2 EF580993 Haliotis_FJ8 $\overline{1} 2176$ Oncorhynchus HSC71 AAB21658 X_maculatus_ $\mathrm{H} S C 70$ A $\mathrm{B} 062115$ Human_HSPA8_HSC71-NP_006588 Rattus HSC7 $\overline{1}$ NM $0 \overline{2} 43 \overline{5} 1$ Pelodis̄cus HS̄C70_HQ219723 Bos HSP70 Ū09861 Humān_HSPĀ 1 A_NM_005345 Rattus_HSP70_1A-NM_031971 X_maculatus_ $\bar{H} S P \overline{7} 0-\overline{1} \_$AB0 62113 $\mathrm{X}$ maculatus HSP70-2 AB0 62114 Hüman_HSPA1 $\bar{L}$ NM $005 \overline{5} 27$ Human HSPA 6 NP 002146 Alligātor_HS̄P70_AB306279

Drosophila_Bb_AF295957 Drosophila_Aa_AF2 95933 Spodoptera_FJ $\overline{7} 54276$ Bombyx_AB0 35326 Paralvinella_EF580992 B_plicatilis_HSP70-1 AB775784 B_plicatilis_HSP70-2_AB775785 A brightwelli $\bar{i}$ HSP70 $\overline{\mathrm{K}} \times 119429$ Wüchereria AF̄̄ 67352 Setaria $\mathrm{AF} \overline{0} 79360$ C_elegañs_HSP70A_M18540 C_elegans_HSP-1_NM_070667 M_nipponeñse_kC̄ $60 \overline{3} 43$ M-nipponense-DQ660140 Mȳtilus HSP70 AY861684 Perinerēis_KŪ255783 Paralvinella HSP70-2 EF580993 Haliotis FJ $8 \overline{1} 2176$ Oncorhynchus_HSC71 AAB21658 $\mathrm{X}$ maculatus $\overline{\mathrm{H}} \mathrm{SC7}$ - $\overline{\mathrm{A}} \mathrm{B} 062115$ Hüman HSPA8 ${ }^{-}$HSC71 NP 006588 Rattus HSC7 $\overline{1}$ NM $0 \overline{2} 43 \overline{5} 1$ Pelodiscus HS̄C $7 \overline{0}$ HQ219723 Bos HSP70 Ū09861 Humān_HSPĀ 1 A_NM_005345 Rattus_HSP70-1A-NM 031971

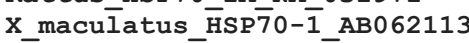
$x$ maculatus HSP70-2 AB0 62114

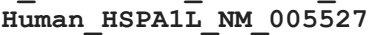
Human HSPA 6 NP 002146 Alligātor_H $\bar{S} P 7 \overline{0} \_$АB 306279
AAACGTAACACCACCATCCCAACCAAGCAGACTCAGACCTTCACCACCTACTCAGACAAC 1302 AAGAGGAACACCACCATCCCCACCAAGCAGACCCAGACCTTCACAACTTACTCCGACAAC 1302 AAGCGTAATACCACCATTCCTACCAAGCAGACACAGACCTTCACTACCTATTCTGACAAC 1302 AAGCGCAATACCACCATTCCCACCAAGCAGACCCAGACTTTCACCACСTACTCTGACAAC 1302 AAGCGGAACACTACAATTCCCACCAAGCAGACTCAGACATTCACCACATACTCTGACAAC 1302 AAGCGCAACTCCACCATCCCCACGAAGCAGACGCAGATCTTCACCACCTACTCGGACAAC 1302 AAGCGCAACTCCACCATCCCCACCAAGCAGACGCAGATCTTCACCACCTACTCCGACAAC 1302 AAGCGCAACTCCACCATCCCCACCAAGCAGACGCAGACCTTCACCACCTACTCGGACAAC 1302 AAGCGAAACACCACCATCCCCACAAAGCAGACTCAGATCTTCTCCACATACTCAGATAAC 1308 AAACGCAACACCACCGTCCCCACCAAGCAGACCCAGGTGTTCAGCACCTACTCCGACAAC 1308 AAGCGCAACTCCACCATCCCCACCAAGCAGACACAGATTTTCACCACCTACTCTGACAAC 1308 CAGAGGAACGCCACTATCCCCACCAAGCAGACCCAGACTTTCACCACCTACTCGGACAAC 1308 AAGCGCAACACCACCATCCCCACCAAGCAGACGCAGACCTTCACCACCTACTCGGACAAC 1311

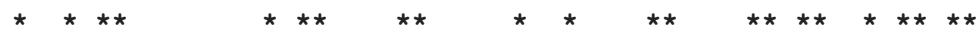

CAGCCCGGAGTCTCCATCCAGGTGTATGAGGGCGAACGTGCGATGACGAAGGACAACAAT 1356 CAGCCCGGAGTCTCCATCCAGGTGTATGAGGGCGAACGTGCGATGACGAAGGACAACAAT 1356 CAGCCAGCGGTCACGATCCAAGTGTACGAGGGCGAGCGAGCGATGACCAAGGACAACAAC 1356 CAGCCGGCCGTCACCATCCAGGTGTACGAGGGAGAGAGAGCGATGACAAAGGACAACAAC 1356 CAGCCTGCAGTGACCATCCAAGTGTACGAGGGCGAGCGAGCGATGACCAAAGACAACAAC 1371 CAGCCCGGCGTGTTGATTCAAGTGTACGAGGGCGAGAGGGCCATGACCAAGGACAATCAT 1362 CAGCCCGGCGTGTTGATTCAAGTGTACGAGGGCGAGAGGGCCATGACCAAGGACAATCAT 1362 CAGCCGGGAGTGTTGATTCAAGTCTACGAAGGCGAAAGAGCCATGACTAAAGACAACAAT 1359 CAGCCTGGTGTCCTTATTCAGGTTTACGAAGGTGAACGTGCTTTGACCAAAGACAATAAC 1362 CAGCCAGGTGTTCTTATTCAGGTTTATGAAGGCGAACGTGCTATGACCAAGGACAATAAT 1362 CAACCAGGAGTGTTGATCCAGGTTTACGAAGGAGAACGTGCCATGACCAAGGACAACAAC 1365 CAACCAGGAGTGTTGATCCAGGTTTACGAAGGAGAACGTGCCATGACCAAGGACAACAAC 1365 CAGCCTGGAGTGCTCATTCAAGTTTACGAAGGAGAACGTGCCATGACAAAAGACAATAAC 1362 CAGCCTGGTGTGTTAATTCAAGTTTATGAAGGAGAGCGTGCAATGACAAAAGATAACAAC 1362 CAGCCTGGTGTATTAATCCAGGTTTATGAAGGAGAGAGAGCTATGACCAAGGACAACAAC 1365 CAGCCAGGTGTGTTGATCCAAGTTTACGAGGGTGAGCGTGCCATGACCAAGGACAACAAC 1359 CAGCCCGGTGTCTTGATCCAGGTGTACGAGGGAGAGCGTGCCATGACCAAAGACAACAAC 1362 CAACCTGGTGTGCTGATTCAGGTATTCGAAGGTGAGAGAGCCATGACAAAGGATAACAAC 1362 CAGCCTGGTGTGCTCATTCAGGTGTATGAGGGTGAGAGGGCCATGACCAAGGACAACAAC 1362 CAGCCTGGCGTGCTCATTCAGGTGTTTGAGGGCGAAAGGGCAATGACCAAAGATAACAAC 1362 CAGCCTGGTGTGCTTATTCAGGTTTATGAAGGCGAGCGTGCCATGACAAAGGATAACAAC 1362 CAGCCAGGTGTACTCATCCAGGTGTATGAAGGTGAAAGGGCCATGACCAAGGACAACAAC 1362 CAGCCTGGTGTACTCATTCAGGTCTACGAAGGGGAGAGAGCCATGACAAAAGACAACAAC 1362 CAGCCGGGCGTGCTGATCCAGGTGTACGAGGGCGAGAGGGCCATGACGCGGGACAACAAC 1362 CAACCCGGGGTGCTGATCCAGGTGTACGAGGGCGAGAGGGCCATGACGAAAGACAACAAT 1362 CAGCCCGGGGTGCTGATCCAGGTGTACGAGGGCGAGAGGGCCATGACGCGCGACAACAAC 1362 CAGCCAGGAGTCCTGATTCAGGTCTATGAAGGTGAGAGAGCCATGACAAAGGACAACAAT 1368 CAGCCCGGGGTCCTGATCCAGGTCTACGAAGGGGAGAGAGCCATGACCAAGGACAACAAC 1368 CAACCCGGGGTGCTGATCCAGGTGTATGAGGGCGAGAGGGCCATGACAAAGGACAACAAC 1368 CAGCCTGGGGTCTTCATCCAGGTGTATGAGGGTGAGAGGGCCATGACCAAGGACAACAAC 1368 CAGAGCAGCGTGCTGGTGCAGGTGTACGAGGGCGAGCGCGCCATGACCAAGGACAACAAC 1371

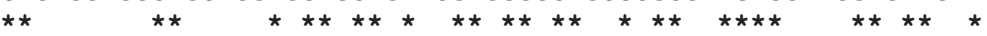

GCATTGGGCACCTTCGATCTGTCCGGCATTCCACCTGCACCAAGGGGTGTGCCCCAGATA 1416 GCATTGGGCACCTTCGATCTGTCCGGCATTCCACCTGCACCAAGGGGTGTGCCCCAGATA 1416 CTGCTGGGCACGTTCGACTTGACCGGCATCCCGCCCGCGCCTCGCGGTGTCCCTAAGATC 1416 СTACTGGGCACGTTCGACCTGACGGGGATCCCGCCCGCGCCGCGAGGAGTGCCCAAGATC 1416 СTCCTCGGACGATTTGAGCTCAGCGGTATTCCGCCAGCTCCACGCGGAATTCCCAAGATC 1431 TTGTTGGGCAAGTTTGAGCTGTCGGGCATACCGCCGGCGCCCCGCGGCGTGCCCCAAATC 1422 TTGTTGGGCAAGTTTGAGCTGTCGGGCATACCGCCGGCGCCCCGCGGCGTGCCCCAAATC 1422 TTACTCGGTAAATTTGAGCTTACTGGCATTCCACCGGCGCCAAGAGGTGTTCCTCAAATC 1419 TTGCTTGGTAAATTCGAGTTGTCTGGGATTCCGCCTGCTCCTCGCGGTGTGCCGCAGATT 1422 TTGCTCGGTAAATTTGAGTTGTCTGGAATCCCGCCTGCTCCTCGTGGTGTGCCACAAATA 1422 TTGCTCGGAAAGTTCGAGCTCTCCGGAATCCCACCAGCACCACGCGGAGTCCCACAAATC 1425 TTGCTCGGAAAGTTCGAGCTCTCCGGAATCCCACCAGCACCACGCGGAGTCCCACAAATC 1425 ATTTTGGGTAAGTTTGAGCTGACTGGTATTCCCCCAGCGCCAAGGGGAGTGCCACAAATC 1422 CTGCTAGGTAAATTGAACTGTCTGGTATTCCTCCAGCTCCACGAGGAGTGCCACAAATT 1422 TTGCTTGGAAAGTTTGAATTAACTGGAATACCTCCAGCACCAAGAGGTGTGCCCCAGATT 1425 СTCTTGGGTAAATTCGAGCTGTCCGGCATTCCСССTGCTCCTCGTGGCGTTCCССAGATC 1419 CTGCTGGGAAAGTTCGAGCTGACTGGCATTCCACCTGCTCCTCGTGGTGTGCCACAGATA 1422 ATCTTGGGCAAGTTTGAGCTGACTGGAATTCCCCCAGCACCCAGAGGTGTCCCACAGATT 1422 CTGTTGGGCAAGTTTGAGCTGACTGGAATCCCCСCTGCACCTCGCGGTGTTCCTCAGATT 1422 СTGCTCGGGAAATTTGAGCTGACGGGAATACCGCCAGCTCCTCGGGGGGTCCCTCAGATT 1422 CTGCTTGGCAAGTTTGAACTCACAGGCATACCTCCTGCACCCCGAGGTGTTCCTCAGATT 1422 CTGCTTGGGAAGTTTGAGCTCACAGGCATACCTCCAGCACCCCGTGGGGTTCCTCAGATT 1422 TTGCTGGGCAAGTTTGAGCTGACTGGCATTCCTCCAGCTCCAAGAGGTGTACCTCAAATT 1422 СTGCTGGGGCGCTTCGAGCTGAGCGGCATCCCGCCGGCCCCGCGGGGGGTGCCCCAGATC 1422 CTGTTGGGGCGCTTCGAGCTGAGCGGCATCCCTCCGGCCCCCAGGGGCGTGCCCCAGATC 1422 CTGCTGGGGCGCTTCGAGTTGAGCGGCATCCCGCCGGCTCCCAGGGGCGTGCCCCAGATC 1422 СTCCTGGGGAAGTTTGAGCTCTCTGGTCTTCCTCCTGCTCCCAGAGGCGTGCCACAGATC 1428 CTGCTGGGCAGGTTTGAGCTGACGGGAATCCCGCCCGCTCCACGAGGGGTCCCGCAGATC 1428 СTGCTGGGGCGGTTTGACCTGACTGGAATCCCTCCAGCACCCAGGGGAGTTCCTCAGATC 1428 CTGCTGGGGCGTTTTGAACTCAGTGGCATCCCTCCTGCCCCACGTGGAGTCCCCCAGATA 1428 СTGCTGGGCAAGTTCGACCTGACCGGCATCCCGCCCGCGCCGCGCGGCGTGCCGCAGATC 1431 
bioRxiv preprint doi: https://doi.org/10.1101/2020.09.21.307264; this version posted April 22, 2021. The copyright holder for this preprint (which was not certified by peer review) is the author/funder, who has granted bioRxiv a license to display the preprint in perpetuity. It is made available under aCC-BY-NC-ND 4.0 International license.

Drosophila_Bb_AF295957 Drosophila_Aa_AF295933 Spodoptera_FJ754276 Bombyx_AB0 35326 Paralvinella_EF580992 B_plicatilis_HSP70-1_AB775784 B_plicatilis_HSP70-2_AB775785 A brightwellii_HSP70_KX119429 Wüchereria_AF'167352 Setaria AF $\overline{0} 79360$ C_elegañs_HSP70A_M1 8540 C-elegans HSP-1 NM 070667 M_nipponense_KC $\overline{4} 60 \overline{3} 43$ M-nipponense-DQ660140 Mytilus_HSP70_AY861684 Perinerēis KU 255783 Paralvinelīa_HSP70-2_EF580993 Haliotis_FJ812176 Oncorhynchus_HSC71_AAB21658 X_maculatus__HSC70_ĀBO62115 Human HSPA8 ${ }^{-}$HSC71 NP 006588 Rattus_HSC7 $\overline{1}$ NM $0 \overline{2} 43 \overline{5} \overline{1}$ Pelodiscus HS C7 Bos_HSP70_Ū09861 Humān_HSPĀ1A_NM 005345 Rattus HSP70 1 A NM 031971 $\mathrm{X}$ maculatus_HSP $\overline{7} 0-\overline{1}$ _AB0 62113 $X$ maculatus HSP70-2 AB062114

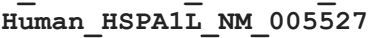
Human HSPA $6 \overline{\mathrm{N} P} \overline{0} 02146$ Alligätor_HS̄P7O__AB306279

Drosophila Bb AF295957 Drosophila_Aa_AF2 95933 Spodoptera FJ $\overline{7} 54276$ Bombyx_AB0 35326 Paralvinella_EF580992 B_plicatilis_HSP70-1_AB775784 B_plicatilis_HSP70-2_AB775785 A brightwelli HSP70 $\overline{\mathrm{K}} \mathrm{X} 119429$ Wüchereria AF'̄ 67352 Setaria AF 079360 C_elegañs_HSP70A_M18540 C-elegans HSP-1 NM 070667 M_nipponeñse_KC $460 \overline{3} 43$ M-nipponense DQ660140 Mȳtilus_HSP70 AY 861684 Perinerēis KŪ255783 Paralvinella HSP70-2 EF580993 Haliotis_FJ $8 \overline{1} 2176$ Oncorhynchus HSC71 AAB21658 $\mathrm{X}$ maculatus_HSC70_ĀB062115 Hüman HSPA8 ${ }^{-}$HSC71 NP 006588

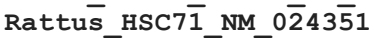
Pelodis̄cus HS̄C70_HQ219723 BOS_HSP70_Ū09861 Humān_HSPĀ 1 A NM_005345 Rattus_HSP70_1A_NM_031971 X_maculatus_. $\mathrm{X}$ maculatus_HSP70-2 AB0 62114 Human_HSPA1 $\overline{\mathrm{L}}$ NM $005 \overline{5} 27$ Human HSPA 6 NP 002146 Alligātor_HS̄P70_AB306279

Drosophila Bb AF295957 Drosophila Aa AF295933 Spodoptera FJ754276 Bombyx_AB0 35326 Paralvinella_EF580992 B plicatilis HSP70-1 AB775784 B_plicatilis_HSP70-2_AB775785 A brightwelli HSP70 $\overline{\mathrm{K}} \mathrm{i} 119429$ Wüchereria $\mathrm{AF} \overline{1} 67352$ Setaria AF 079360 C_elegañs_HSP70A_M18540
GAAGTAACCTTCGACTTGGACGCCAATGGAATCCTGAACGTCAGCGCCAAGGAGATGAGC 1476 GAAGTTACCTTCGACTTGGACGCCAATGGAATCCTGAACGTCAGCGCCAAGGAGATGAGC 1476 GACGTCACTTTCGACCTGGACGCGAACGGCATTCTCAACGTGTCGGCCAAAGAGAACAGC 1476 GACGTGACGTTCGACATGGACGCTAACGGCATCCTGAACGTGTCGGCCAAGGAGAACAGC 1476 GACGTCACATTCGACATCGACGCCAACGGAATCCTACACGTGACGGCCAAAGACCAAAGT 1491 GAGGTCACATTTGACATTGATGCCAATGGTATTCTGAATGTGTCCGCCGCAGACAAATCG 1482 GAGGTCACATTTGACATTGATGCCAATGGTATTCTGAATGTGTCCGCTGCAGACAAATCG 1482 GAGGTCACTTTTGACATCGATGCCAACGGTATTTTGAACGTTAGCGCTTGTGATAAGTCA 1479 GAAGTCGACTTTGATATTGATGCCAACGGTATTCTGAATGTTTCCGCCCAGGATAAATCC 1482 GAAGTTACTTTTGATATTGATGCAAACGGTATCTTGAATGTTTCTGCGCAAGACAAATCT 1482 GAAGTCACTTTCGATATTGACGCCAACGGAATCTTGAACGTCTCTGCCACTGACAAGTCC 1485 GAAGTCACTTTCGATATTGACGCCAACGGAATCTTGAACGTCTCTGCCACTGACAAGTCC 1485 GAAGTAACATTTGATATTGATGCTAATGGTATTCTAAATGTATCTGCTGCTGACAAGTCC 1482 GAAGTAACTTTTGATATTGATGCCAATGGTATCTTGAATGTATCTGCTGCAGACAAGTCA 1482 GAAGTGACCTTTGACATTGATGCCAATGGTATCCTGAATGTATCTGCAGTAGATAAGAGT 1485 GAGGTTACCTTCGATATTGATGCCAACGGTATCCTGAATGTCACTGCTGCGGACAAGAGC 1479 GAGGTCACATTCGATATCGATGCCAATGGTATCCTTAACGTGTCTGCTGTAGACAAGAGT 1482 GAAGTAACСTTTGATATTGATGCCAACGGTATCCTCAATGTATCAGCTGTAGACAAGAGC 1482 GAGGTCACATTTGACATTGATGCTAACGGCATCATGAACGTGTCTGCTGCTGACAAGAGC 1482 GAGGTGACATTCGATATCGACGCTAACGGCATCATGAACGTCTCCGCTGTCGACAAGAGC 1482 GAAGTCACTTTTGACATTGATGCCAATGGTATACTCAATGTCTCTGCTGTGGACAAGAGT 1482 GAGGTTACTTTTGACATTGATGCCAATGGCATCCTCAATGTTTCTGCTGTAGATAAGAGC 1482 GAAGTAACATTTGATATTGATGCCAATGGCATCCTGAATGTGTCTGCTGTGGACAAGAGC 1482 GAGGTGACCTTCGACATCGACGCCAATGGCATCCTGAACGTCACGGCCACGGACAAGAGC 1482 GAGGTGACCTTCGACATCGATGCCAACGGCATCCTGAACGTCACGGCCACGGACAAGAGC 1482 GAGGTGACCTTCGACATCGACGCCAACGGCATCCTGAACGTCACGGCCACTGACAAGAGC 1482 GAAGTTACCTTTGATATCGACGCCAACGGCATCCTAAATGTGTCTGCCGTCGACAAAAGC 1488 GAGGTCACCTTTGACGTGGACGCCAACGGCATCCTGAACGTGTCCGCGGTGGACAAAAGC 1488 GAGGTGACGTTTGACATTGATGCCAATGGTATTCTCAATGTCACAGCCACGGACAAGAGC 1488 GAGGTGACTTTTGACATTGATGCTAATGGCATCCTGAGCGTGACAGCCACTGACAGGAGC 1488 GAGGTCACTTTCGACATCGACGCCAACGGCATCCTCAACGTGAGCGCGGTCGACAAGAGC 1491

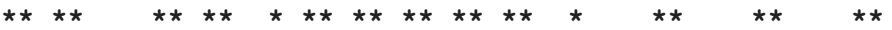

ACGGGCAAGGCCAAGAACATCACGATCAAGAACGACAAGGGACGCCTCTCGCAGGCCGAG 1536 ACGGGCAAGGCCAAGAACATCACGATCAAGAACGACAAGGGACGGCTCTCGCAGGCCGAG 1536 ACCGGCCGAAGCAAGAACATCGTGATCAAGAACGACAAGGGACGCCTGTCGCAGGCCGAG 1536 ACCGGCCGCAGCAAGAACATCGTGATCAAGAACGACAAGGGTCGTCTCTCGCAAGCGGAG 1536 ACTGGTAGGTCAAGTGATATCCACATCAAGAACGAGAAAGGTCGTTTGTCACAGGCCGAA 1551 ACTGGCAAGACGAACAAAATCACCATCACCAATGACAAGGGAAGACTTAGCAAAGAGGAG 1542 ACTGGCAAGACGAACAAAATCACCATCACCAATGACAAGGGAAGACTTAGCAAAGAGGAC 1542 ACTGGCAAACAGAACAAGATCACGATCACGAACGACAAAGGAAGACTCAGCAAAGAGGAC 1539 ACAGGCAAACAAAACAAAATTACCATTACCAATGATAAAGGACGTTTGTCAAAGGATGAG 1542 ACTGGTAAACAAAATAAGATAACTATTACTAATGACAAGGGAAGGTTATCGAAGGATGAG 1542 ACCGGAAAGGCAAAACAGATCACCATCACCAACGACAAGGATCGCTTTTCCAAGGATGAC 1545 ACCGGAAAGCAAAACAAGATCACCATCACCAACGACAAGGGACGTCTTTCCAAGGATGAC 1545 ACAGGCAAAGAGAACAAGATCACCATCACTAATGATAAGGGCCGCTTGAGCAAGGAAGAG 1542 ACTGGTAAGGAGAACAAAATCACAATCACAAATGACAAGGGTCGTCTCAGCAAAGAAGAA 1542 ACTGGTAAAGAGAACAAAATCACCATCACTAACGACAAAGGTCGTTTGAGCAAAGAAGAA 1545 ACCGGCAAGGAGAACAAGATTACCATCACCAACGACAAAGGCCGTCTCAGTAAGGAAGAT 1539 ACTGGTAGAGAGAACAAGATCACTATCACCAATGATAAGGGTCGTCTAAGCAAAGAGGAG 1542 ACTATGAAAGAGAACAAGATCACCATCACAAACGACAAAGGTCGCTTGTCGAAGGAAGAG 1542 ACTGGGAAGGAGAACAAGATCACCATCACAAATGACAAGGGTCGCCTGAGCAAGGAGGAC 1542 ACCGGGAAGGAGAACAAAATCACCATCACAAACGACAAGGGCCGCTTGAGCAAAGAGGAT 1542 ACGGGAAAAGAGAACAAGATTACTATCACTAATGACAAGGGCCGTTTGAGCAAGGAAGAC 1542 ACAGGAAAGGAGAACAAGATCACCATCACCAATGACAAGGGCCGCTTGAGTAAGGAGGAT 1542 ACTGGCAAGGAGAACAAGATCACCATTACCAATGACAAAGGGCGATTAAGCAAGGAAGAC 1542 ACGGGCAAGGCCAACAAGATCACCATCACCAACGACAAGGGCCGGCTGAGCAAGGAGGAG 1542 ACCGGCAAGGCCAACAAGATCACCATCACCAACGACAAGGGCCGCCTGAGCAAGGAGGAG 1542 ACCGGCAAGGCCAACAAGATCACCATCACCAACGACAAGGGCCGCCTGAGCAAGGAGGAG 1542 ACGGGCAAAGAAAACAAAATCACAATCACCAACGACAAGGGCCGACTCAGCAAAGATGAG 1548 ACCGGCAAAGAGAACAAGATCACCATCGCCAACGACAAGGGCCGACTGAGCAAAGACGAG 1548 ACCGGCAAGGTGAACAAGATCACCATCACCAATGACAAGGGCCGCCTGAGCAAGGAGGAG 1548 ACAGGTAAGGCTAACAAGATCACCATCACCAATGACAAGGGCCGGCTGAGCAAGGAGGAG 1548 ACGGGCAAGGAGAACAAGATCACCATCACCAACGACAAGGGCCGGCTCAGCAAGGACGAC 1551

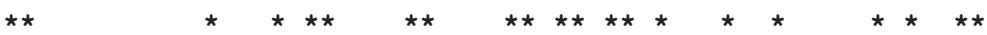

ATTGATCGCATGGTGAACGAGGCTGAGAAGTACGCCGACGAGGACGAAAAGCATCGCCAG 1596 ATTGATCGCATGGTGAACGAGGCTGAAAAGTACGCCGACGAGGACGAGAAGCATCGCCAG 1596 ATCGAGCGCATGCTCGCCGAGGCCGAGAAGTACAAGGACGAGGACGAGAAGCAGAGGCAG 1596 ATCGATCGCATGCTGTCCGAGGCAGAGCGGTACAAGGAGGAAGACGAGAAGCAGAGACAG 1596 ATCGACAGGATGTTGGCCGAAGCTGAGAAATATCGCGAAGAAGACGAGAAACAACGAGAA 1611 ATCGACCGAATGGTCAACGAGGCCGAGAAGTACAAGAAGGATGACGAGGAGCAGAGAGAT 1602 ATCGATCGAATGGTCAATGAGGCCGAGAAGTACAAGAAAGATGACGAGGAGCAGAGAGAT 1602 ATTGAGAGAATGGTCAACGATGCCGAGAAGTATAAAAAAGACGACGAGCAACAAAGAGAG 1599 ATCGAGCGGATGGTACAGGAAGCTGAGAAATACAAGGCGGATGATGAAGCACAGAAGGAT 1602 ATCGAACGGATGGTGCAAGAAGCTGAGAAATATAAGGCAGATGATGAAGCTCAGAAAGAC 1602 ATTGAACGCATGGTCAACGAAGCTGAGAAATACAAGGCTGACGATGAGGCCCAAAAGGAC 1605 
bioRxiv preprint doi: https://doi.org/10.1101/2020.09.21.307264; this version posted April 22, 2021. The copyright holder for this preprint (which was not certified by peer review) is the author/funder, who has granted bioRxiv a license to display the preprint in perpetuity. It is made available under aCC-BY-NC-ND 4.0 International license.

C elegans HSP-1 NM 070667

M_nipponense_KC $\overline{4} 60 \overline{3} 43$

Mnipponense-DQ660140

Mytilus_HSP70_AY861684

Perinereis KU2555783

Paralvinelía_HSP70-2_EF580993

Haliotis_FJ81̄2176

Oncorhynchus_HSC71_AAB21658

$\mathrm{X}$ maculatus $\overline{\mathrm{H} S C 70} \overline{\mathrm{A}} \mathrm{B} 062115$

Hüman_HSPA8_HSC71_NP_006588

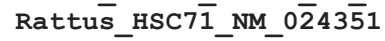

Pelodiscus_HSC70__HQ219723

Bos_HSP70_Ū09861

Human HSPĀ 1 A NM 005345

Rattus_HSP70_1A_NM_031971

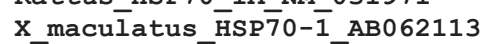

X_maculatus_HSP70-2_AB062114

Human HSPA1 $\bar{L}$ NM $005 \overline{5} 27$

Human_HSPA6_N $\mathrm{N}$ - 002146

Alligator_HS̄P70_AB306279

Drosophila_Bb_AF295957

Drosophila_Aa_AF295933

Spodoptera_FJ754276

Bombyx_AB0 35326

Paralvinella_EF580992

B_plicatilis_HSP70-1_AB775784

B_plicatilis_HSP70-2_AB775785

A_brightwellī_HSP70_KX119429

Wüchereria_AF'̄ 67352

Setaria_AF $\mathbf{0} 79360$

C_elegañs_HSP70A_M18540

C-elegans_HSP-1 NM 070667

M_nipponense_KC $\overline{4} 60 \overline{3} 43$

M-nipponense_DQ660140

Mytilus_HSP70_AY861684

Perinereis KU 255783

Paralvinelīa_HSP70-2_EF580993

Haliotis_FJ8 $\mathbf{1} 2176$

Oncorhynchus_HSC71_AAB21658

X_maculatus_ $\mathrm{HSC70} \overline{\mathrm{A} B} 062115$

Human HSPA8 HSC71 NP 006588

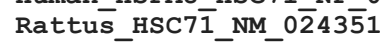

Pelodiscus_HSC70_HQ219723

BOS_HSP70_Ū09861

Human HSPĀ 1 A NM 005345

Rattus_HSP70_1A_NM_031971

X_maculatus_HSP $\overline{7} 0-\overline{1}$ _AB0 62113

X_maculatus_HSP70-2_AB062114

Hüman_HSPA1 $\overline{\mathrm{L}}$ NNM_0055 27

Human HSPA $6 \overline{\mathrm{N}} \mathrm{P} \overline{0} 02146$

Alligātor_HS̄P70_AB306279

Drosophila Bb AF295957

Drosophila Aa AF295933

Spodoptera FJ754276

Bombyx_AB0 $\overline{3} 5326$

Paralvinella_EF580992

B plicatilis HSP70-1 AB775784

B_plicatilis_HSP70-2_AB775785

A brightwellì HSP70 $\bar{K} X 119429$

Wüchereria_AF̄̄ 67352

Setaria AF 79360

C_elegañs_HSP70A_M18540

C elegans HSP-1 NM 070667

M nipponense KC $\overline{4} 60 \overline{3} 43$

M_nipponense DQ660140

Mȳtilus HSP70 AY861684

Perineréis_KU 255783

Paralvinelīa_HSP70-2_EF580993

Haliotis_FJ8̄̄ 2176

Oncorhynchus_HSC71 AAB21658

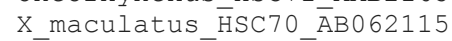

Human HSPA8_HSC71_NP_006588

Rattus_HSC7 $\overline{1}$ NM $0 \overline{2} 43 \overline{5} 1$

Pelodiscus HS C7 $\overline{0}$ HQ219723

BOS_HSP70_Ū09861
ATTGAACGCATGGTCAACGAAGCTGAGAAATACAAGGCTGACGATGAGGCCCAAAAGGAC 1605 ATTGAGCGGATGGTGCAAGAGGCCGAGAAGTACAAAGCTGACGATGAGAAACAGCGGGA 1602 ATTGAACGTATGGTTCAAGAAGCTGAAAAGTACAAAGCTGATGATGAGAAGCAACGTGAT 1602 ATTGAACGCATGGTCAATGATGCTGAGAAATACAAGGCAGAAGACGAGAAACAGAAGGAC 1605 ATTGACCGCATGGTTAACGAAGCCGAGCGTCTCAAGGCTGAAGATGATGCGCAGAGAGAA 1599 ATTGAACGTATGGTCAAGGATGCTGAGAGGTACAAGCAAGAAGATGAAAGTCAGAAGGAT 1602 ATTGAGCGAATGGTTAATGAGGCAGAGAACTACAAGGCCGAGGATGAGAAGCAGAAGGAT 1602 ATTGAGCGCATGGTCCAGGAGGCTGAGAAGTACAAGTGTGAGGATGATGTGCAGCGTGAC 1602 ATTGAGCGCATGGTTCACGAAGCGGAGACATACAGGGCTGAAGACGACTTGCAGCGAGAA 1602 ATTGAACGTATGGTCCAGGAAGCTGAGAAGTACAAAGCTGAAGATGAGAAGCAGAGGGAC 1602 ATTGAGCGCATGGTCCAAGAAGCTGAGAAGTACAAAGCTGAGGATGAGAAGCAGAGAGAT 1602 ATTGAACGCATGGTGCAGGAAGCAGAAAAATACAAAGCAGAAGATGAGAAACAGCGTGAC 1602 ATCGAGCGCATGGTGCAGGAGGCGGAAAAGTACAAGGCGGAGGACGAGGTGCAGCGCGAG 1602 ATCGAGCGCATGGTGCAGGAGGCGGAGAAGTACAAAGCGGAGGACGAGGTGCAGCGCGAG 1602 ATCGAGCGCATGGTGCAGGAGGCCGAGCGCTACAAGGCGGAGGACGAGGTGCAGCGCGAG 1602 ATCGAGAAGATGGTGCAGGATGCAGACAAGTACAAGGCGGAGGATGACCAGCAGAGAGAA 1608 ATCGAGAGGATGGTGCAGGACGCCGAGAAGTACAAAGCCGAGGACGAGCTGCAGAGGGAC 1608 ATTGAGCGCATGGTTCTGGATGCTGAGAAATATAAAGCTGAAGATGAGGTCCAGAGGGAG 1608 GTGGAGAGGATGGTTCATGAAGCCGAGCAGTACAAGGCTGAGGATGAGGCCCAGAGGGAC 1608 ATCGACCGCATGGTGCAGGAGGCCGAGAAGTACAAGGCGGAGGACGACGCCAACCGCGAG 1611

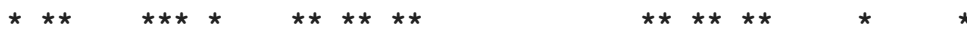

CGCATAACCTCTAGAAATGCTCTGGAGAGCTACGTATTCAACGTAAAGCAGTCCGTGGAG 1656 CGAATAACCTCTAGAAATGCCCTGGAGAGCTACGTCTTCAATGTGAAGCAGGCCGTGGAA 1656 CGCGTAGCGTCTCGGAACCAGCTCGAGTCGTACGTGTTCAGCGTGAAGCAGGCGCTGGAC 1656 CGCGTGGCCGCTCGGAACCAGCTCGAATTGTATTTGTTCAGCGTGAAGCAGGCGCTCGAC 1656 CGTATCTCGGCCAGAAACAGCTTGGAACAATACATCTACTCATACAAAGAGGCCGCTACT 1671 AAGGTGGCCGCCAAGAACTCGCTTGAGTCCTACTGCTTTAACATGAAACAAACGGTGGAG 1662 AAGGTGGCCGCCAAGAACTCGCTTGAGTCCTACTGCTTTAACATGAAACAAACGGTGGAG 1662 AGAATCACTGCCAAGAACTCGTTGGAATCCTACTGCTTCAACATGAAACAAACCATCGAG 1659 CGTATTGCGGCAAAAAATGCTCTGGAATCGTACGCATTCAATATGAAACAAACGATCGAG 1662 CGTATTGCGGCAAAGAATGCTCTTGAATCATATGCGTTCAATATGAAGCAAACAATTGAG 1662 CGTATTGGAGCCAAGAACGGACTCGAGTCATACGCCTTCAACCTTAAGCAGACCATTGAG 1665 CGTATTGGAGCCAAGAACGGACTCGAGTCATACGCCTTCAACCTTAAGCAGACCATTGAG 1665 CGTATCTCTGCCAAGAACAACCTGGAGTCTTACTGTTTCAATATGAAGTCCACTGTTGAA 1662 CGCATTGCAGCAAAGAATAGTTTGGAATCTTACTGCTTCAACATGAAATCCACAGTGGAA 1662 CGTATCACCGCCAAAAATAGTCTAGAAAGCTACTCATTTAACATGAAACAAACAGTTGAA 1665 CGTATCACAGCCAAGAACCAGCTGGAGAGCTATGCGTTCAACATGAAATCAACTGTCGAG 1659 CGCATCCAAGCCAAGAATGCCCTGGAGAGCTATGCCTTTAACATGAAGTCGACTGTCGAG 1662 CGCATCCAGGCCAAGAACGGTTTGGAAAGCTATGCCTTCAACATGAAGTCGACTGTAGAG 1662 AAGGTCTCTTCTAAGAACTCCCTAGAGTCCTACGCTTTCAACATGAAGTCTACTGTGGAG 1662 AAGGTGGTGGCCAAGAACAGCCTGGAGTCCTACACTTTCAACATGAGGTCCACCATAGAG 1662 AAGGTGTCATCCAAGAATTCACTTGAGTCCTATGCCTTCAACATGAAAGCAACTGTTGAA 1662 AAGGTTTCCTCTAAGAACTCGCTGGAGTCTTATGCTTTCAACATGAAAGCAACTGTTGAG 1662 AAAGTTTCCTCTAAGAATTCTCTAGAATCATATGCCTTTAATATGAAGGCTACAGTTGAA 1662 AGGGTGTCTGCCAAGAACGCGCTGGAGTCGTACGCCTTCAACATGAAGAGCGCCGTGGA 1662 AGGGTGTCAGCCAAGAACGCCCTGGAGTCCTACGCCTTCAACATGAAGAGCGCCGTGGA 1662 AGGGTGGCTGCCAAGAATGCGCTCGAGTCCTATGCCTTCAACATGAAGAGCGCCGTGGAG 1662 AAGATCGCGGCAAAGAATTCCCTGGAGTCCTACGCTTACCACATGAAGAGCAGCGTCGAA 1668 AAGATAGCTGCCAAGAACTCCCTGGAGTCCTACGCTTTCACCGTGAAGAGCAGCGTGGAG 1668 AAAATTGCTGCAAAGAATGCCTTAGAATCCTATGCTTTTAACATGAAGAGTGTTGTGAGT 1668 AGAGTGGCTGCCAAAAACTCGCTGGAGGCCCATGTCTTCCATGTGAAAGGTTCTTTGCAA 1668 CGGGTGGCGGCCAAGAACTCGCTCGAGTCCTACGCCTACAACATGAAGCAGACGGTGGAG 1671

CAGGCGCC---CGCTGGCAAACTGGACGAGGCCGACAAGAACTCCGTCCTGGACAAGTGC 1713 CAGGCACC---TGCTGGCAAATTGGACGAGGCTGACAAGAACTCCGTCTTGGACAAGTGC 1713 GAGGCCGG-----AGACAAGCTCTCGGAACAGGACAAGAGCACGGCGAGGGACGCGTGC 1710 GAGGCCGG------CGACAAACTGAGCGACGCGGACAAGAGCACGGCGCGCGACGCGTGT 1710 GAGGC---CGATAGTTCTAAACTGTCACAGACTGATAAGGATCAAGTGATCAACAAATGT 1728 GACGAAAAGCTGGCTGCTAAGATTAGTGCCGACGACAAGAAGAAGATTTTGGACGCGTGT 1722 GACGAAAAGCTGGCTGCTAAGATTAGTGCAGATGACAAGAAGAAGATTTTGGACGCGTGT 1722 GACGAGAAATTGGCAGCTAAAGTCTCGGCTGATGAAAAGAAGAAGATTTTGGACGCGTGC 1719 GACGAGAAGTTAAAAGATAAAATTTGGAAGAGGATAAGAAAAAAATTCAAGAAAAATGC 1722 GATGAAAAGCTGAGGGACAAACTATCTGAGGAGGATAAGAAGAAGATCCAGGAGAAGTGT 1722 GACGAGAAGCTCAAGGATAAGATCAGCCCAGAAGACAAGAAGAAGATCGAGGACAAGTGC 1725 GACGAGAAGCTCAAGGATAAGATCAGCCCAGAAGACAAGAAGAAGATCGAGGACAAGTGC 1725 GATGACAAATTCAAGGATAAGGTTCCTGAGGATGATCGCAACAAGATCATGGAAGCCTGT 1722 GATGACAAGTTTAAGGACAAGGTGCCAGAGGAGGATCGCAACAAGATAATGGAAGCTTGC 1722 GATGAAAAACTCAAGGATAAAATCAGTGAAAGTGACAAAAAGGAAATCATGGACAAATGT 1725 GACGAGAAACTGAAGGACAAGATTTCCGACGAGGACAAGACCAAGATCACTGAGAAATGC 1719 GACGAGAAATTGAAGGACAAGATCAGTGATGCTGATAAGAAGACCATTACAGACAAATGT 1722 GATGAGAAACTGAAGGACAAGATCAGTGAAGATGACAAGAAAACCATCACTGACAAGTGC 1722 GATGAGAAACTGCAGGGGAAAATCAGTGACGAGGACAAGACTAAGATTCTGGAGAAGTGC 1722 GACGAGAAGCTGAAGGATAAGATCAGCGAATCGGACAAGAAGAAGGTCCTGGACAAATGC 1722 GATGAGAAACTTCAAGGCAAGATTAACGATGAGGACAAACAGAAGATTCTGGACAAGTGT 1722 GATGAGAAACTTCAAGGCAAGATCAATGATGAAGACAAACAGAAGATTCTTGACAAGTGC 1722 GATGAGAAGCTCCAGGGCAAGATCAGCAGTGAAGACAAGCAGAAAATCCTAGACAAATGT 1722 GATGAGGGGCTGAAGGGCAAGATCAGCGAGGCGGACAAGAAGAAGGTGCTGGACAAGTGC 1722 
bioRxiv preprint doi: https://doi.org/10.1101/2020.09.21.307264; this version posted April 22, 2021. The copyright holder for this preprint (which was not certified by peer review) is the author/funder, who has granted bioRxiv a license to display the preprint in perpetuity. It is made available under aCC-BY-NC-ND 4.0 International license.

Human HSPA1A NM 005345 Rattus HSP70 1A NM 031971 $X$ maculatus $\bar{H} S P \overline{7} 0-\overline{1}$ AB062113 X_maculatus_HSP70-2_AB062114

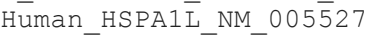
Human_HSPA6 N Alligātor_HS̄P7 $\overline{0} \_$AB306279

Drosophila_Bb_AF295957 Drosophila Aa-AF295933 Spodoptera FJ754276 Bombyx_AB0 35326 Paralvinella EF580992 B_plicatilis_HSP70-1_AB775784 B plicatilis HSP70-2 AB775785 A_brightwelli_HSP70_KX119429 Wüchereria AFĪ 67352

Setaria AF079360

C_elegañs_HSP70A_M18540 C elegans HSP-1 NM 070667 M_nipponense_KC $\overline{4} 60 \overline{3} 43$ M-nipponense DQ660140 Mytilus_HSP70_AY861684 Perinerēis KŪ 55783 Paralvinelīa_HSP70-2_EF580993 Haliotis FJ81̄2176 Oncorhynchus_HSC71_AAB21658 $X$ maculatus_ $\mathrm{HSC70} \overline{\mathrm{A}} \mathrm{B} 062115$ Human HSPA 8 HSC71 NP 006588 Rattus_HSC7 $\overline{1}$ NM_ $0 \overline{2} 43 \overline{5} 1$ Pelodiscus HS̄C7 $\overline{0}$ HQ219723 BOS_HSP70_Ū09861 Humān HSPĀ1A NM 005345 Rattus HSP70 1A NM 031971 $\mathrm{X}$ maculatus $\overline{\mathrm{H} S P} \overline{7} 0-\overline{1}$ AB0 62113 X_maculatus_HSP70-2_AB062114 Hüman HSPA1 $\overline{\mathrm{L}}$ NM $005 \overline{5} 27$ Human HSPA 6 N P 002146 Alligātor_HS̄P7 $\overline{0}$ AB306279

Drosophila Bb AF295957 Drosophila_Aa_AF295933 Spodoptera FJ $\overline{7} 54276$ Bombyx_AB0 $\overline{3} 5326$ Paralvinella EF580992 B_plicatilis_HSP70-1_AB775784 B plicatilis HSP70-2 AB775785 A brightwelli HSP70 KX119429 Wuchereria AF167352 Setaria AF079360 C_elegañs_HSP70A_M18540 C elegans HSP-1 NM 070667 M_nipponense_KC $\overline{4} 60 \overline{3} 43$ M nipponense DQ660140 Mȳtilus_HSP70_AY861684 Perinerēis KŪ255783 Paralvinelīa_HSP70-2_EF580993 Haliotis FJ8 $\overline{1} 2176$ Oncorhynchus_HSC71_AAB21658 $\mathrm{X}$ maculatus $\overline{\mathrm{H} S C 70} \overline{\mathrm{AB}} 062115$ Human HSPA ${ }^{-}{ }^{-H S C 71}{ }^{-}$NP 006588 Rattus_HSC7 $\overline{1}$ NM_0 $243 \overline{5} 1$ Pelodiscus HSC7̄ HQ219723 Bos_HSP70_Ū09861 Humān HSPĀ1A NM 005345 Rattus HSP70 1A NM 031971 X_maculatus_HSP $\overline{7} 0-\overline{1} \_A B 062113$ $X$ maculatus_HSP70-2_AB062114 Human HSPA1 $\overline{\mathrm{L}}$ NM $005 \overline{5} 27$ Human HSPA 6 N P 002146 Alligātor_HS̄PP $\overline{0}$ _AB306279

Drosophila Bb AF295957 Drosophila_Aa_AF295933 Spodoptera-FJ754276 Bombyx_AB0 $\overline{3} 5326$
GATGAGGGGCTCAAGGGCAAGATCAGCGAGGCGGACAAGAAGAAGGTGCTGGACAAGTGT 1722 GACGAGGGTCTCAAGGGCAAGATCAGCGAGGCTGACAAGAAGAAGGTGCTGGACAAGTGC 1722 GACGACGCCTTGAAAGGAAAAATCAGTGAGGAGGAGAAAAAGCTGGTTATTGACAAATGC 1728 GACGAGAGCTTGAAGGGCAAGATCAGCCTTAGAGGACAAGAAGAAGGTGTGAACAAGTGC 1728 GATGAAGGTTTGAAGGGCAAGATTAGTGAGTCTGATAAAAATAAAATATTGGATAAATGC 1728 GAGGAAAGCCTTAGGGACAAGATTCCCGAAGAGGACAGGCGCAAAATGCAAGACAAGTGT 1728 GACGAGAAGCTGGCGGGCAAGATCGGCGACCAGGACAAGCAGCGTGTGCTCGACAAGTGC 1731

AACGAAACTATTCGATGGCTGGACAGCAACACCACCGCCGAGAAGGAGGAGTTCGACCAC 1773 AACGACACTATCCGGTGGCTGGACAGCAACACCACCGCCGAGAAGGAGGAGTTCGACCAC 1773 GACGACGCGCTCAAGTGGCTCGACAACAACACGCTGGCGGAGCAGGAGGAGTACGAGCAC 1770 GACGAGGCGCTGCGGTGGCTGGACAACAACACTCTCGCTGACCAGGATGAATACGAGCAC 1770 GAGCAGACTGTTAAGTGGTTGGATAACAACACTTTGGCAGAAAAAGAGGAATACGAACAT 1788 GAAGAGGCCTTGAAATGGCTGGACTCGAACCAGACCGCTGAGAAGGACGAGTTCGAGCAC 1782 GAAGAGGCCTTGAAATGGCTGGACTCGAACCAGACCGCTGAGAAGGACGAGTTCGAGCAC 1782 GAGAACGCGCTCAAATGGCTGGACGCGAATCAAACGGCCGAGAAGGATGAGTTTGAGCAT 1779 GACGAAACAGTTAGGTGGTTGGATGGAAATCAAACAGCTGAAAAAGATGAATTTGAGCAT 1782 GATGAGACGGTCAGGTGGTTGGATGGGAATCAGACAGCTGAGAAGGATGAGTTTGAGCAT 1782 GACGAGATCTTGAAGTGGCTCGACAGCAACCAGACCGCAGAGAAGGAGGAGTTCGAGTCA 1785 GACGAGATCTTGAAGTGGCTCGACAGCAACCAGACCGCAGAGAAGGAGGAGTTCGAGCAC 1785 AATGATGCCATCAAGTGGCTCGACACTAATCAGCTTGGGGAGAAGGAAGAATATGAACAC 1782 AATGATGCCATCAAATGGCTGGATACTAACCAGCTGGGAGAGAAGGAAGAATATGAACAT 1782 GACGAAATCATTAAATGGTTGGATGCCAACAATCTGGCTGAGAAGGAAGAATTTGAACAC 1785 AACGAGGTCATCAGGTGGTTGGACTCCAACCAGACCGCAGAGAAGGACGAGTTTGAACAC 1779 AACGATATCATAGCTTGGCTGGATGCTAACCAGCTCGCTGAAAAAGATGAATTTGAACAC 1782 AACGATGTTATCAGCTGGTTAGACTCTAACCAGTTGGCTGAGAAGGATGAGTTCGAACAC 1782 AACGAGGTCATCGGGTGGCTGGACAAGAACCAGACTGCTGAGAAGGAAGAGTATGAGCAC 1782 AACGAGGTCATCAGCTGGCTCGACAAAAATCAGAGTGCAGAGAAAGAGGAGTTTGAGCAC 1782 AATGAAATTATCAACTGGCTTGATAAGAATCAGACTGCTGAGAAGGAAGAATTTGAACAT 1782 AACGAAATCATCAGCTGGCTGGATAAGAACCAGACTGCGGAGAAGGAAGAATTTGAGCAT 1782 AACGAAATTATCAACTGGCTGGATAAGAATCAGACGGCTGAAAAAGAAGAGTTTGAACAT 1782 CAGGAGGTGATTTCCTGGCTGGACGCCAACACCTTGGCGGAGAAGGACGAGTTTGAGCAC 1782 CAAGAGGTCATCTCGTGGCTGGACGCCAACACCTTGGCCGAGAAGGACGAGTTTGAGCAC 1782 CAGGAGGTCATCTCCTGGCTGGACTCTAACACGCTGGCTGAGAAAGAGGAGTTCGTGCAC 1782 AACCAGACAATTTCCTGGCTGGAGAACAACCAGCTGGCAGAGAAGGAGGAGTATGAACAT 1788 CAGGAGACCATCAGCTGGCTGGAGAACAACCAGCTGGCTGAGAAAGACGAGTACCAACAC 1788 AACGAGCTCCTTTCGTGGCTGGAGGTCAATCAACTGGCAGAGAAAGATGAGTTTGATCAT 1788 CGGGAAGTCCTTGCCTGGCTGGAGCACAACCAGCTGGCAGAGAAGGAGGAGTATGAGCAT 1788 CAGGAGCTGATCGCCTGGCTGGACCGCAACCAGATGGCCGAGAAGGACGAGTACGAGCAC 1791

AAGATGGAGGAGCTCACTCGCCACTGTTCCCCTATCATGACCAAGATGCATCAGCAGGGA 1833 AAGATGGAGGAGCTCACTCGCCACTGCTCCCCTATCATGACCAAGATGCATCAGCAGGGA 1833 AAGCTGAAGGACGTGCAGCGCGTCTGCTCGCCCGTCATGGCCAAGATGCATGGGGCGGGC 1830 AAGCTAAAGGATGTGCAGCGAGTGTGTTCGCCGGTCATGAGCAAGATGCACGGTGCGGCG 1830 CATTTGGAGGAGTTACACAAGGTCTGTTCTCCGATAATGGCCAAGCTACATGGATCACAG 1848 AAGATGAAAGAAGTGGAGAAAATTTGCTCGCCGATTATTACTCAGTTGTATCAGGGCGCT 1842 AAGATGAAAGAAGTGGAGAAAATTTGCTCGCCGATTATTACTCAGTTGTATCAGGGCGCT 1842 AAGTTGAAGGAGATCGAGAAGACTTGTTCGCCGATCATTACCAAGTTGTATCAGGGTGGT 1839 CGCCAAAAAGAATTGGAATCTGTTTGCAATCCGATTATTACAAAACTCTACCAGAGCGCT 1842 CGTCAGAAAGAGTTGGAAGCAGTTTCGAATCCAATCATTACGAAACTGTACCAGAGTGCT 1842 CAACAGAAGGATTTGGAAGGATTGGCCAAGCCCGATCTTTCCAAGCTTTACCAGAGTGCC 1845 CAACAGAAGGATTTGGAAGGATTGGCCAACCCAATCATTTCCAAGCTTTACCAGAGTGCC 1845 AAACTGAAGGAAATTGAACAGATATGTAATCCTATCATCACCAAAATGTACCAAGCAGCT 1842 AAGTTGAAGGAAATTGAGCAAATATGTAATCCTATCATCACCAAAATGTACCAAGCAGCT 1842 AAACAGAAAGAACTTGAGGGAGTGTGTAATCCAATCATCACTAAACTGTACCAGTCTGCT 1845 CAACAGAAGGAATTGGAAGGCATCTGCATGCCTATCATCACCAAGTTGTACCAGGCAGGT 1839 CAACAAAAGGAACTGGAGAAGATCTGTATGCCGATCATTACAAAGCTGTACCAGGCAGGT 1842 AAGCAGAAGGAGCTGGAGGGTGTGTGTAATCCAATCATCACCAAGCTGTACCAGGCAGCT 1842 CACCAGAAGGAGTTGGAGAAGGTGTGCAACCCCATCATCACCAAGCTGTACCAGGGTGCT 1842 CAGCAGAAGGAGCTTGAGAAGTTGTGCAACCCGATTATGACCAAACTGTACCAGAGTGAA 1842 CAACAGAAAGAGCTGGAGAAAGTTTGCAACCCCATCATCACCAAGCTGTACCAGAGTGCA 1842 CAGCAGAAAGAACTGGAGAAGGTCTGCAACCCTATCATCACCAAGCTGTACCAGAGTGCT 1842 CAGCAGAAGGAGCTGGAGAAGGTCTGCAATCCCATAATCACAAAGCTGTACCAGAGCGCT 1842 AAGAGGAAGGAGCTGGAGCAGGTGTGTAACCCCATCATCAGCAGACTGTACCAGGGGGCG 1842 AAGAGGAAGGAGCTGGAGCAGGTGTGTAACCCCATCATCAGCGGACTGTACCAGGGTGCC 1842 AAGCGGGAGGAGCTGGAGCGGGTGTGCAACCCGATCATCAGCGGGCTGTATCAGGGTGCG 1842 CAGCAGAATGAGCTGGAGAGGGTGTGCAAACCGGTCGTGGCAAAGTTGTATCAAGGTGCA 1848 CAGCAGAAAGAGCTGGAGAAGGTGTGCAACCCCATCATCAGCAACTTGTACCAGGGAGGG 1848 AAGAGAAAGGAATTGGAGCAGATGTGTAACCCTATCATCACAAAACTCTACCAAGGAGGA 1848 CAGAAGAGGGAGCTGGAGCAAATCTGTCGCCCCATCTTCTCCAGGCTCTATGGGGGGCCT 1848 AAGCAGAAGGAGCTGGAGAAGATTTGCAACCCCATCATCACCAAGCTATACCAAGGCGGC 1851

GCGGGAGCAGCTGGGGGTCCGGGA---GCCAACTGTGGCCAACAGGCCGGAGGATTTGGC 1890 GCGGGAGCAGCTGGGGGTCCGGGA---GCCAACTGTGGCCAACAGGCCGGAGGATTTGGC 1890 GCGCAAGGTGCGGGCGGGATGCCCGGAGGTATGCCTGGTGGCATGCCCGGAGGAATGCCT 1890 CCCGGCGGTATG--------CCAGGAGGAATGCCGGGCGGTATGCCGGGAGGATACCAA 1881 
bioRxiv preprint doi: https://doi.org/10.1101/2020.09.21.307264; this version posted April 22, 2021. The copyright holder for this preprint (which was not certified by peer review) is the author/funder, who has granted bioRxiv a license to display the preprint in perpetuity. It is made

Paralvinella EF580992 B_plicatilis_HSP70-1_AB775784 B plicatilis HSP70-2 AB775785 A_brightwellí_HSP70_K̄X119429 Wüchereria AF'̄67352

Setaria AF079360 C_elegañs HSP70A M18540 C elegans HSP-1 NM 070667 M_nipponense_KC $\overline{4} 60 \overline{3} 43$ M nipponense DQ660140 Mÿtilus_HSP7O Perineréis KU 255783

Paralvinelía_HSP70-2_EF580993 Haliotis FJ8 $\overline{1} 2176$

Oncorhynchus_HSC71_AAB21658 $\mathrm{X}$ maculatus $\overline{\mathrm{H} S C 70} \overline{\mathrm{A}} \mathrm{B} 062115$ Human_HSPA8_HSC71_NP_006588 Rattus HSC7 $\overline{1}$ NM $0 \overline{2} 43 \overline{5} 1$ Pelodiscus HS $\overline{\mathrm{S}} 7 \overline{0}$ HQ219723 BOS HSP70 Ü09861 Human_HSPĀ1A NM 005345 Rattus_HSP70_1A_NM_031971 $X$ maculatus $\bar{H} S P \overline{7} 0-\overline{1}$ AB062113 X_maculatus_HSP70-2_AB062114

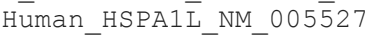
Human ${ }^{-}$HSPA 6 NP 002146 Alligātor H $\overline{\mathrm{S}} \mathrm{P} 7 \overline{0}$ AB306279

Drosophila Bb_AF295957 Drosophila Aa AF295933 Spodoptera FJ754276 Bombyx_AB0 $\overline{3} 5326$ Paralvinella EF580992 B_plicatilis_HSP70-1_AB775784 B plicatilis HSP70-2 AB775785 A_brightwelli_HSP70_KX119429 Wüchereria AF̄ 67352

Setaria AF079360 C_elegans HSP70A M18540 C elegans HSP-1 NM 070667 M_nipponense_KC $\overline{4} 60 \overline{3} 43$ M nipponense DQ660140 Mȳtilus_HSP70_AY861684 Perinereis KU 255783

Paralvinelīa_HSP70-2_EF580993 Haliotis FJ812176

Oncorhynchus_HSC71_AAB21658 $X$ maculatus $\overline{\mathrm{H} S C 70} \overline{\mathrm{AB}} 062115$ Human_HSPA8_HSC71_NP_006588 Rattus $\bar{s}_{\text {_HSC7 }} \overline{1}$ NM $0 \overline{2} 43 \overline{5} 1$ Pelodiscus_HS̄C70_HQ219723 BOS_HSP70_Ū09861 Humān HSPĀ 1 A NM 005345 Rattus_HSP70_1A_NM_031971 $X$ maculatus $\bar{H} S P \overline{7} 0-\overline{1}$ AB0 62113 X_maculatus_HSP70-2_AB062114 Hüman HSPA1 $\overline{\mathrm{L}}$ NM $005 \overline{5} 27$ Human HSPA 6 NP 002146 Alligator_HS̄P7 $\overline{0}$ _AB306279

Drosophila Bb AF295957 Drosophila_Aa_AF295933 Spodoptera ${ }^{-} \mathrm{F} \overline{7} 54276$ Bombyx_AB0 $\overline{3} 5326$ Paralvinella EF580992 B_plicatilis_HSP70-1_AB775784 B plicatilis HSP70-2 AB775785 A_brightwellí_HSP70_KX119429 Wuchereria AF167352 Setaria AF079360 C_elegañs_HSP70A_M18540 C elegans HSP-1 NM 070667 M_nipponense_KC $\overline{4} 60 \overline{3} 43$ M-nipponense DQ660140 Mytilus_HSP7 $\overline{0}$ AY861684 Perineréis KŪ255783 Paralvinelīa_HSP70-2_EF580993 available under aCC-BY-NC-ND 4.0 International license.

TCTGGATCTTGTGGTGGTTCCGGATCACAACAAGGTTGTTACGGATCACAGACTGG---A 1905 GGTGGTGCGCCTGGTGGCATGCCTGGTGGTATGC------------CTGGCGGTATGCCT 1890 GGTGGTGCGCCTGGTGGTATGCCTGGTGGTATGCCTGGTGGTATGCCGGGCGGTATGCCT 1902 GCGCCGGGCGGTATGCCGGGTGGTATGCCGGGCGGTGCTGGTGC---------------T 1884 GGTGGTATGCCTGGAGGGATGCCGGGTGGCATGCCTAGTGGAGCTCCAGGTGCTGGTTCA 1902 GGTGGTATGCCCGGAGGAATGCCTGGTGGTATGCCTGGTGGAGCTCCAGGTGGAGGTTCG 1902 GGAGGAGCCCCACCAGGTGCTGCTCCAGGAGGAGCCGCCGGAGGAGC------------ 1892

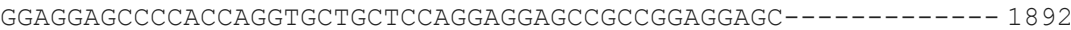
GGTGGTGCTCCTCCAGGTGGTATGCCAGGAGGTTTTCCAGGTGCCCCAGGGGCTGGTGCT 1902 GGTGGTGCTCCTCCAGGTGGTATGCCAGGAGGTTTTCCAGGTGCCCCAGGGGCTGGTGCT 1902 GGTGGAGCCCCAGGTGGTGGTATGCCAAACTTTGGTGGAGCTGGTGGAGCCCCAGGAGGT 1905 GGCGCCCCTGCTGGTGGAATGCCCGGTGGAATGCCCGGTGGAATGCCCGGAGGCTTCCCA 1899 GGTGCTCCACCAGGCAGTATGCCAGGTGGCATGCCCGGTGGTATGCCAGGAGGCGCTGGT 1902 GGCGGTGCTGGTGGTATGCCCAACTTCAACCCAGGTGCTGCTGGTGCTGGCGGTGCAGGA 1902 GGTGGGATGCCCGGCGGTATGCCTGAGGGCATGGCTGGCGGATTCCCTGGAGCTGGTGGT 1902 GGACAGATGCCTAGAGCTGGTGCGACCGGAACAGGAGGGGCCTCGGGCCCCACCA----- 1897 GGAGGCATGCCAGGAGGAATGCCTGGGGGATTTCCTGGTGGTGGAGCTCCTCCCTCTG-- 1900 GGTGGCATGCCTGGAGGAATGCCTGGTGGCTTCCCTGGTGGAGGAGCTCCTCCATCTG-- 1900 GGAGGAATGCCTGGTGGCATGCCTGGGGGATTCCCTGGAGGTGGAGCTGCCCCATCTG-- 1900 GGCGGCCCCGGGGCTGGCGGCTTTGGGGCTCAGGGCCCTAAAGGGGGC---TCTGGGTCT 1899 GGTGGTCCCGGGCCTGGGGGCTTCGGGGCTCAGGGTCCCAAGGGAGGG---TCTGGGTCA 1899 GGTGCTCCCGGGGCTGGGGGCTTCGGGGCCCAGGCGCCCAAGGGAGGC---TCTGGGTCG 1899

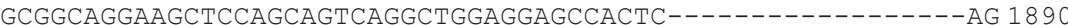
AGGCCCACTGGGAGCTGCAGAGAGCAGGCAGGAGCCCACA--------------GCCAG 1893 TGCACTGGGCCTGCCTGCGGAACAGGGTATGTGCCTG------GAAGG---CCTGCCACA 1899 GGTGTCCCTGGGGGCAGCAGTTGTGGCACTCAAGCCCGCCAGGGGGAC---CCCAGCACC 1905

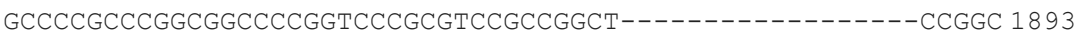

GG------CTACTCTGGACCCACAGTCGAGGAGGTCGACTAA---n---n---n---- 1926 GG------CTACTCTGGACCCACAGTCGAGGAGGTCGACTAA------------------ 1926 GGAGGAATGCCGGGCGGAATGCCTGGTGGCATGCCCGGCGGTATGCCAGGAGGAATGCCT 1950 CAAGCGAGGAGTGATGGACCCACAGTTGAGGAAGTCGACTAA------------------ 1923

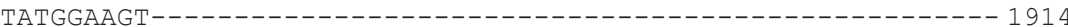

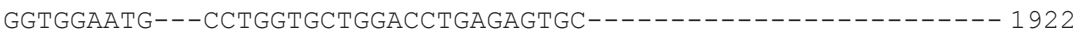

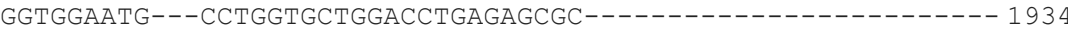

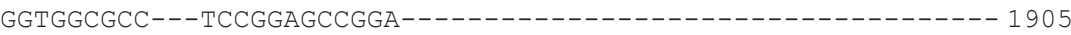

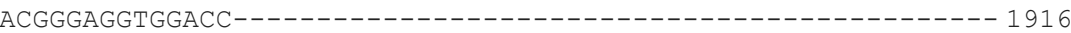

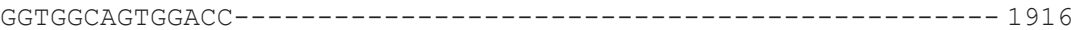

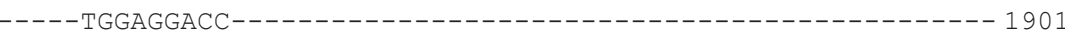

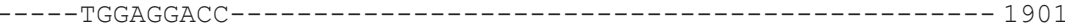
GCTCCTG------GT------------------GG----------------------- 1913

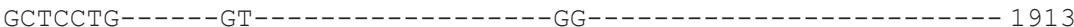
GCACCTGGAG---CA---------GGAGGCACTGG------------------------ 1928

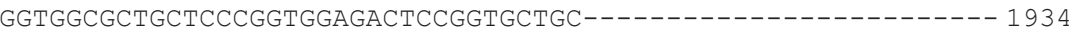

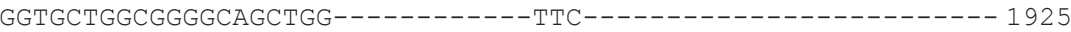

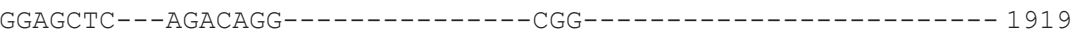

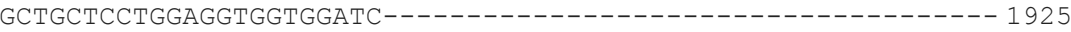
-

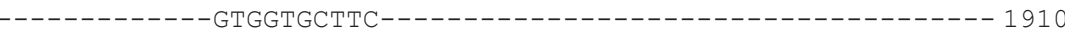

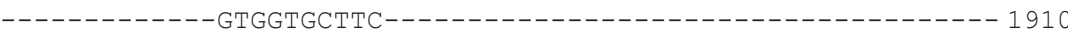

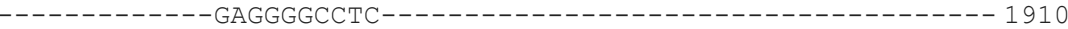

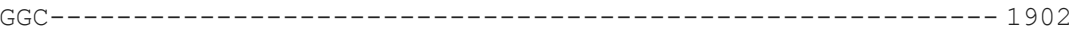
GGC---- - - - - - -

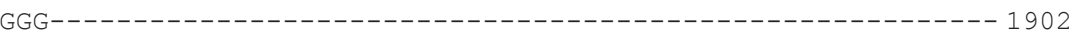

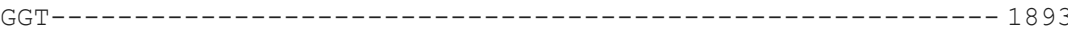

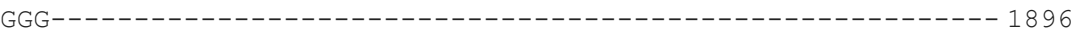

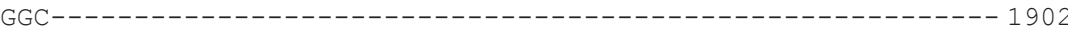

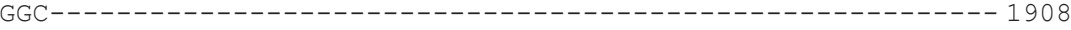

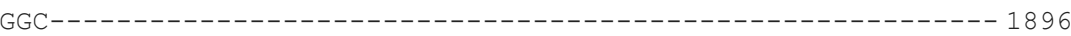

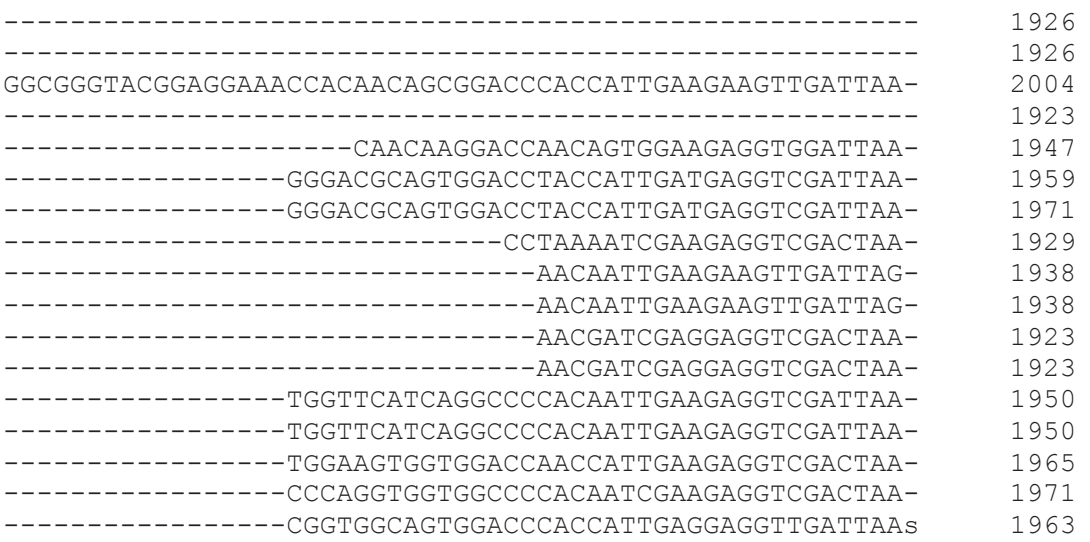


bioRxiv preprint doi: https://doi.org/10.1101/2020.09.21.307264; this version posted April 22, 2021. The copyright holder for this preprint (which was not certified by peer review) is the author/funder, who has granted bioRxiv a license to display the preprint in perpetuity. It is made available under aCC-BY-NC-ND 4.0 International license.

Haliotis FJ812176

Oncorhynchus_HSC71 AAB21658

$\mathrm{X}$ maculatus $\overline{\mathrm{H}} \mathrm{SC} 70 \overline{\mathrm{A}} \mathrm{B} 062115$

Hüman HSPA ${ }^{-}{ }^{-} \mathrm{HSC}^{-}{ }^{-} \mathrm{NP} 006588$

Rattus HSC $7 \overline{1}$ NM $0 \overline{2} 43 \overline{5} 1$

Pelodiscus_HS̄C70_HQ219723

Bos HSP70 Ū09861

Humān_HSPĀ 1 A NM 005345

Rattus_HSP70_1A_NM_031971

$\mathrm{X}$ maculatus $\overline{\mathrm{H} S P} \overline{7} 0-\overline{1}$ AB0 62113

$X$ maculatus HSP70-2 AB0 62114

Human HSPA1 $\bar{L}$ NM $005 \overline{5} 27$

Human_HSPA 6_NP_ō02146

Alligator_H $\bar{S} P 7 \overline{0}$ AB306279

\begin{tabular}{|c|}
\hline 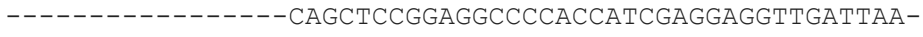 \\
\hline ---------------------ATCTGGACCAACCATTGAGGAAGTCGACTAA- \\
\hline 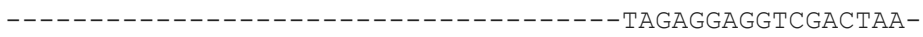 \\
\hline----------------------- CTCAGGGCCCACCATTGAAGAGGTTGATTAA - \\
\hline ---------------------TTCAGGCCCCACCATTGAAGAGGTCGATTAA- \\
\hline----------------------- ATCTGGACCAACCATTGAGGAAGTAGATTAA - \\
\hline ---------------------------CCCACCATTGAGGAGGTGGATTAG- \\
\hline -----------------------------CCCACCATTGAGGAGGTAGATTAG- \\
\hline------------------------------ CCCACCATCGAGGAGGTGGATTAG - \\
\hline------ CCCACTGTCGAGGAGGTCGACTAA- \\
\hline ------CCCACTGTGGAGGAGGTGGACTGA- \\
\hline ---CCCACAATTGAAGAAGTAGATTAA- \\
\hline --CCCATCATTGAGGAGGTTGATTGA- \\
\hline 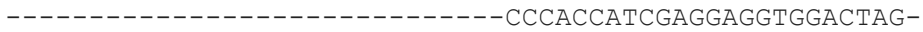 \\
\hline
\end{tabular}

Supplementary fig. S5. Comparison of HSP70 nucleotide sequences used for the calculation of synonymous and nonsynonymous substitution rates. Regions shown in bold were used for the calculation. 


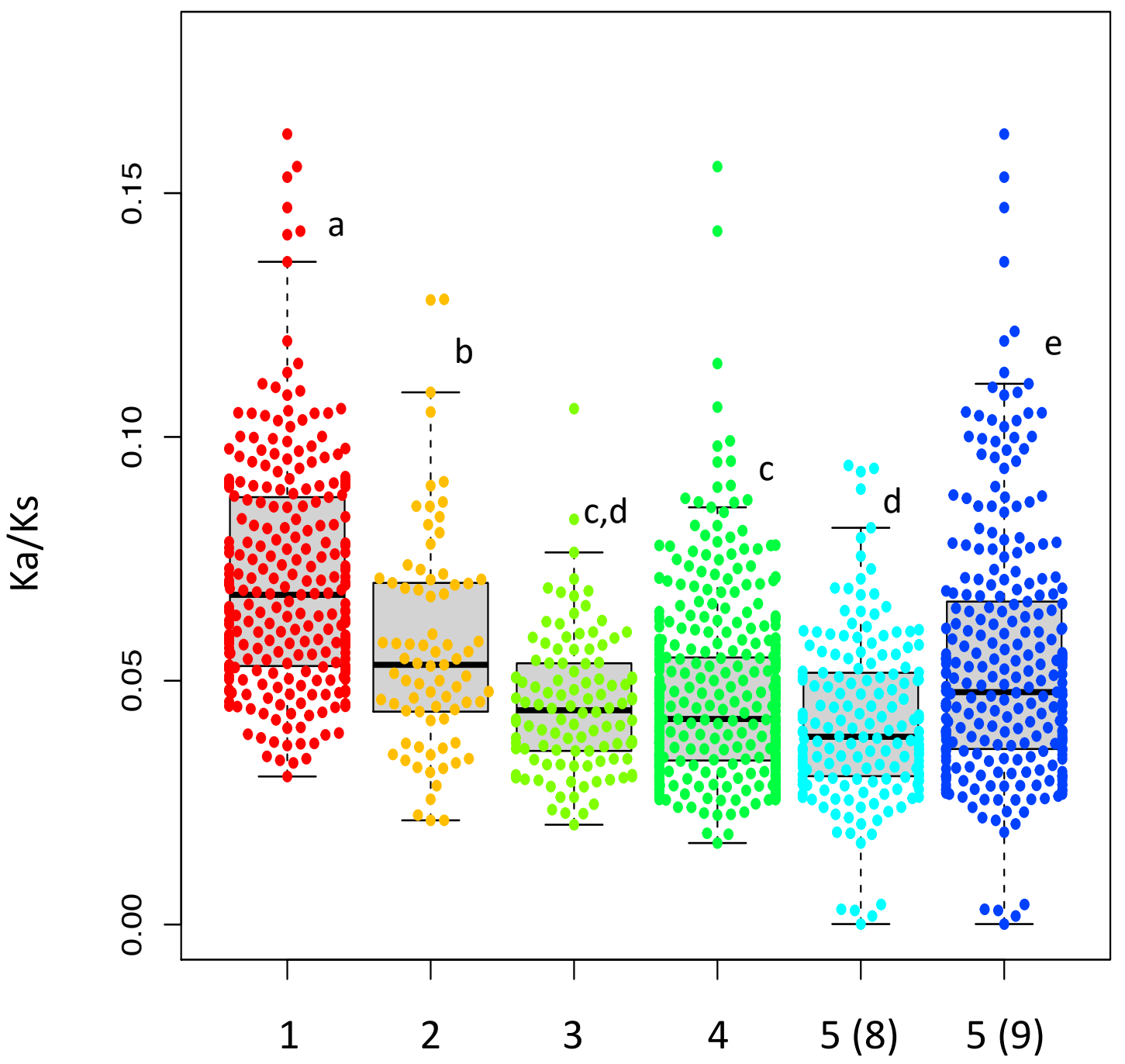

Clade (node)

1: Invertebrate HSP70s (Lineage A)

2: Rotifera HSP70s (Lineage B)

3: Nematode HSP70s (Lineage B)

4: Invertebrate HSP/HSC70s (Lineage B)

5 (8): Vertebrate HSC70s (Lineage B)

5 (9): Vertebrate HSP70s (Lineage B)

Supplementary fig. S6. Bee swarm boxplots of $\mathrm{Ka} / \mathrm{Ks}$ values for each clade. Only $\mathrm{Ka} / \mathrm{Ks}$ values calculated from inter-cluster pairs were averaged. Statistical differences were calculated by Kruskal-Wallis test (chi-squared $=200.45$, df $=5$, p-value $<2.2 \mathrm{e}-16$ ) followed by the nonparametric post-hoc tests (pairwise Wilcox test with $P$ value adjustment by the Holm method). Clades sharing same letters are not significantly different at the $5 \%$ level of significance. One outlier in clade $3(0.53)$ is not included in the plot although this value was used for all statistical analyses. 


\begin{tabular}{|c|c|c|c|c|c|c|c|c|c|c|c|c|c|c|c|c|c|c|c|c|c|c|c|c|c|c|c|c|c|c|c|c|}
\hline \multirow{3}{*}{\multicolumn{2}{|c|}{$\frac{\text { Gene No. }}{2}$}} & & \multicolumn{5}{|c|}{ Clade 1} & \multicolumn{3}{|c|}{ Clade 2} & \multicolumn{4}{|c|}{ Clade 3} & \multicolumn{6}{|c|}{ Clade 4} & \multicolumn{5}{|c|}{ Clade 5 (node 8) } & \multicolumn{7}{|c|}{ Clade 5 (node 9) } \\
\hline & & & 1 & 2 & 3 & 4 & 5 & 6 & 7 & 8 & 9 & 10 & 11 & 12 & 13 & 14 & 15 & 16 & 17 & 18 & 19 & 20 & 21 & 22 & 23 & 24 & 25 & 26 & 27 & 28 & 29 & 30 \\
\hline & & D. melanogaster HSP70Aa (AF295933) & 0.00 & & & & & & & & & & & & & & & & & & & & & & & & & & & & & \\
\hline \multirow{3}{*}{$\begin{array}{l}\frac{\overrightarrow{0}}{0} \\
\frac{\pi}{0}\end{array}$} & 3 & Spodoptera HSP70 (FJ754276) & 0.09 & 0.09 & & & & & & & & & & & & & & & & & & & & & & & & & & & & \\
\hline & 4 & Bombyx HSP70 (АВ035326) & 0.10 & 0.10 & 0.03 & & & & & & & & & & & & & & & & & & & & & & & & & & & \\
\hline & 5 & Paralvinella HSP70-1 (EF580992) & 0.16 & 0.16 & 0.14 & 0.14 & & & & & & & & & & & & & & & & & & & & & & & & & & \\
\hline \multirow{3}{*}{$\frac{\pi}{\frac{0}{0}}$} & 6 & B. plicatilis_HSP7O-1 (AB775784) & 0.15 & 0.15 & 0.14 & 0.14 & 0.15 & & & & & & & & & & & & & & & & & & & & & & & & & \\
\hline & 7 & B. plicatilis_HSP70-2 (AB775785) & 0.15 & 0.15 & 0.14 & 0.14 & 0.15 & 0.00 & & & & & & & & & & & & & & & & & & & & & & & & \\
\hline & 8 & A. brightwelli_HSP70 (KX119429) & 0.16 & 0.15 & 0.15 & 0.16 & 0.15 & 0.06 & 0.06 & & & & & & & & & & & & & & & & & & & & & & & \\
\hline \multirow{5}{*}{$\begin{array}{l}\frac{n}{0} \\
\frac{\tilde{d}}{0} \\
\end{array}$} & 9 & Wuchereria (AF167352) & 0.16 & 0.15 & 0.15 & 0.16 & 0.16 & 0.08 & 0.09 & 0.09 & & & & & & & & & & & & & & & & & & & & & & \\
\hline & 10 & Setaria (AF079360) & 0.15 & 0.15 & 0.15 & 0.15 & 0.16 & 0.08 & 0.08 & 0.09 & 0.01 & & & & & & & & & & & & & & & & & & & & & \\
\hline & 11 & C. elegans HSP70A (M18540) & 0.16 & 0.16 & 0.16 & 0.16 & 0.18 & 0.09 & 0.09 & 0.11 & 0.05 & 0.05 & & & & & & & & & & & & & & & & & & & & \\
\hline & 12 & C. elegans HSP-1 (NM_070667) & 0.15 & 0.15 & 0.15 & 0.15 & 0.17 & 0.08 & 0.08 & 0.09 & 0.04 & 0.03 & 0.01 & & & & & & & & & & & & & & & & & & & \\
\hline & 13 & M. nipponense HSP70-1 (КС460343) & 0.16 & 0.15 & 0.15 & 0.15 & 0.15 & 0.07 & 0.07 & 0.08 & 0.08 & 0.08 & 0.09 & 0.08 & & & & & & & & & & & & & & & & & & \\
\hline & 14 & M. nipponense HSP7O-2 (DQ660140) & 0.15 & 0.15 & 0.15 & 0.15 & 0.15 & 0.07 & 0.07 & 0.07 & 0.08 & 0.08 & 0.09 & 0.08 & 0.01 & & & & & & & & & & & & & & & & & \\
\hline & 15 & Mytilus HSP70 (AY861684) & 0.16 & 0.16 & 0.16 & 0.16 & 0.15 & 0.09 & 0.09 & 0.07 & 0.09 & 0.09 & 0.11 & 0.10 & 0.07 & 0.07 & & & & & & & & & & & & & & & & \\
\hline & 16 & Perinereis HSP70-1 (KU255783) & 0.15 & 0.15 & 0.17 & 0.16 & 0.15 & 0.09 & 0.09 & 0.08 & 0.10 & 0.10 & 0.11 & 0.10 & 0.07 & 0.07 & 0.06 & & & & & & & & & & & & & & & \\
\hline & 17 & Paralvinella HSP70-2 (EF580993) & 0.17 & 0.17 & 0.17 & 0.17 & 0.16 & 0.10 & 0.10 & 0.09 & 0.11 & 0.11 & 0.12 & 0.11 & 0.08 & 0.08 & 0.07 & 0.06 & & & & & & & & & & & & & & \\
\hline & 18 & Haliotis HSP70c (FJ812176) & 0.15 & 0.15 & 0.16 & 0.16 & 0.16 & 0.10 & 0.10 & 0.09 & 0.10 & 0.10 & 0.12 & 0.11 & 0.08 & 0.08 & 0.06 & 0.06 & 0.07 & & & & & & & & & & & & & \\
\hline \multirow{6}{*}{ 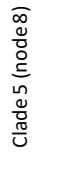 } & 19 & Oncorhynchus HSC71 (AAB21658) & 0.16 & 0.16 & 0.16 & 0.16 & 0.16 & 0.08 & 0.08 & 0.08 & 0.09 & 0.09 & 0.11 & 0.09 & 0.06 & 0.06 & 0.07 & 0.06 & 0.07 & 0.08 & & & & & & & & & & & & \\
\hline & 20 & X. maculatus HSC70 (AB062115) & 0.18 & 0.17 & 0.16 & 0.16 & 0.15 & 0.09 & 0.09 & 0.08 & 0.11 & 0.11 & 0.12 & 0.11 & 0.07 & 0.07 & 0.07 & 0.08 & 0.09 & 0.08 & 0.04 & & & & & & & & & & & \\
\hline & 21 & Human_HSPA8 (NP_006588) & 0.15 & 0.15 & 0.14 & 0.15 & 0.15 & 0.07 & 0.07 & 0.07 & 0.09 & 0.09 & 0.10 & 0.09 & 0.07 & 0.06 & 0.06 & 0.07 & 0.07 & 0.07 & 0.03 & 0.05 & & & & & & & & & & \\
\hline & 22 & Rattus HSC71 (NM_024351) & 0.15 & 0.15 & 0.14 & 0.15 & 0.15 & 0.07 & 0.07 & 0.07 & 0.09 & 0.09 & 0.10 & 0.09 & 0.06 & 0.06 & 0.06 & 0.07 & 0.07 & 0.07 & 0.03 & 0.05 & 0.00 & & & & & & & & & \\
\hline & 23 & Pelodiscus HSC70 (HQ219723) & 0.15 & 0.15 & 0.14 & 0.15 & 0.14 & 0.07 & 0.07 & 0.07 & 0.09 & 0.08 & 0.10 & 0.09 & 0.06 & 0.06 & 0.06 & 0.07 & 0.07 & 0.06 & 0.03 & 0.04 & 0.00 & 0.00 & & & & & & & & \\
\hline & 24 & Bos HSP70 (U09861) & 0.15 & 0.15 & 0.15 & 0.15 & 0.16 & 0.10 & 0.10 & 0.09 & 0.10 & 0.10 & 0.12 & 0.11 & 0.09 & 0.09 & 0.09 & 0.10 & 0.11 & 0.10 & 0.08 & 0.09 & 0.07 & 0.07 & 0.07 & & & & & & & \\
\hline \multirow{7}{*}{ 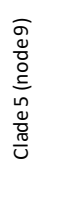 } & 25 & Human_HSPA1A (NM_005345) & 0.15 & 0.15 & 0.15 & 0.15 & 0.15 & 0.10 & 0.09 & 0.09 & 0.10 & 0.09 & 0.11 & 0.10 & 0.08 & 0.08 & 0.09 & 0.09 & 0.11 & 0.10 & 0.07 & 0.09 & 0.06 & 0.06 & 0.06 & 0.00 & & & & & & \\
\hline & 26 & Rattus HSP70_1A (NM_031971) & 0.15 & 0.15 & 0.15 & 0.15 & 0.16 & 0.10 & 0.10 & 0.09 & 0.10 & 0.10 & 0.12 & 0.11 & 0.09 & 0.09 & 0.09 & 0.09 & 0.11 & 0.10 & 0.08 & 0.09 & 0.07 & 0.07 & 0.07 & 0.01 & 0.01 & & & & & \\
\hline & 27 & X. maculatus HSP70-1 (AB062113) & 0.17 & 0.17 & 0.17 & 0.16 & 0.16 & 0.10 & 0.10 & 0.09 & 0.11 & 0.10 & 0.12 & 0.11 & 0.09 & 0.09 & 0.09 & 0.10 & 0.09 & 0.10 & 0.07 & 0.08 & 0.06 & 0.06 & 0.06 & 0.07 & 0.07 & 0.07 & & & & \\
\hline & 28 & X. maculatus HSP70-2 (AB062114) & 0.16 & 0.16 & 0.16 & 0.17 & 0.16 & 0.10 & 0.10 & 0.09 & 0.11 & 0.10 & 0.12 & 0.11 & 0.09 & 0.09 & 0.09 & 0.10 & 0.11 & 0.10 & 0.08 & 0.09 & 0.07 & 0.07 & 0.07 & 0.07 & 0.07 & 0.07 & 0.06 & & & \\
\hline & 29 & Human_HSPA1L (NM_005527) & 0.15 & 0.15 & 0.15 & 0.16 & 0.16 & 0.10 & 0.10 & 0.10 & 0.10 & 0.10 & 0.12 & 0.11 & 0.09 & 0.09 & 0.10 & 0.10 & 0.11 & 0.11 & 0.09 & 0.10 & 0.08 & 0.08 & 0.08 & 0.04 & 0.04 & 0.04 & 0.07 & 0.07 & & \\
\hline & 30 & Human_HSPA6 (NP_002146) & 0.16 & 0.16 & 0.16 & 0.17 & 0.17 & 0.12 & 0.12 & 0.11 & 0.12 & 0.12 & 0.14 & 0.13 & 0.12 & 0.11 & 0.11 & 0.11 & 0.13 & 0.12 & 0.12 & 0.12 & 0.10 & 0.10 & 0.10 & 0.07 & 0.07 & 0.07 & 0.12 & 0.11 & 0.09 & \\
\hline & 31 & Alligator_HSP70 (AB306279) & 0.16 & 0.16 & 0.16 & 0.16 & 0.17 & 0.09 & 0.09 & 0.08 & 0.10 & 0.10 & 0.12 & 0.10 & 0.09 & 0.09 & 0.09 & 0.09 & 0.10 & 0.10 & 0.08 & 0.09 & 0.07 & 0.07 & 0.07 & 0.09 & 0.09 & 0.09 & 0.08 & 0.09 & 0.10 & 0.11 \\
\hline
\end{tabular}

\begin{tabular}{|c|c|c|}
\hline \multicolumn{3}{|c|}{ Gene No. } \\
\hline \multirow{4}{*}{$\begin{array}{l}\frac{\vec{d}}{0} \\
\frac{\pi}{U}\end{array}$} & 2 & D. melanogaster HSP70Aa (AF295933) \\
\hline & 3 & Spodoptera HSP70 (FJ754276) \\
\hline & 4 & Bombyx HSP70 (AB035326) \\
\hline & 5 & Paralvinella HSP70-1 (EF580992) \\
\hline \multirow{3}{*}{$\begin{array}{l}\frac{\widetilde{d}}{\tilde{O}} \\
\frac{\pi}{U}\end{array}$} & 6 & B. plicatilis_HSP70-1 (AB775784) \\
\hline & 7 & B. plicatilis_HSP70-2 (AB775785) \\
\hline & 8 & A. brightwelli_HSP70 (KX119429) \\
\hline \multirow{5}{*}{$\begin{array}{l}\frac{m}{0} \\
\frac{\tilde{d}}{u}\end{array}$} & 9 & Wuchereria (AF167352) \\
\hline & 10 & Setaria (AF079360) \\
\hline & 11 & C. elegans HSP70A (M18540) \\
\hline & 12 & C. elegans HSP-1 (NM_070667) \\
\hline & 13 & M. nipponense HSP70-1 (КС460343) \\
\hline & 14 & M. nipponense HSP70-2 (DQ660140) \\
\hline & 15 & Mytilus HSP70 (AY861684) \\
\hline & 16 & Perinereis HSP70-1 (KU255783) \\
\hline
\end{tabular}

\begin{tabular}{|c|c|c|c|c|c|c|}
\hline \multicolumn{5}{|c|}{ Clade 1} & \multicolumn{2}{|c|}{ Clade 2} \\
\hline 1 & 2 & 3 & 4 & 5 & 6 & 7 \\
\hline 0.09 & & & & & & \\
\hline 1.48 & 1.48 & & & & & \\
\hline 1.44 & 1.44 & 0.84 & & & & \\
\hline & & 2.83 & & & & \\
\hline 2.23 & 1.80 & 1.50 & 2.48 & & & \\
\hline 2.11 & 1.75 & 1.48 & 2.98 & & 0.01 & \\
\hline & & & 4.43 & 2.41 & 1.93 & 1.90 \\
\hline
\end{tabular}

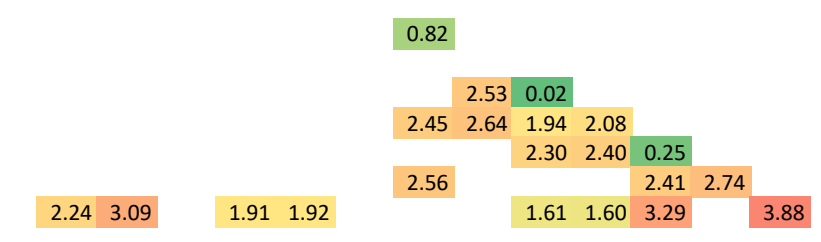




\begin{tabular}{|c|c|c|c|c|c|c|c|c|c|c|c|c|c|c|c|c|c|c|c|c|c|c|c|c|c|c|c|c|c|c|c|c|}
\hline & 17 & Paralvinella HSP70-2 (EF580993) & 1.91 & 1.94 & 2.17 & 2.64 & & 1.99 & 1.95 & & 3.34 & 2.35 & & & 1.69 & 1.72 & 2.13 & 1.61 & & & & & & & & & & & & & & \\
\hline \multirow{6}{*}{ 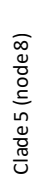 } & 18 & Haliotis HSP70c (FJ812176) & & & & & & & & & & & 1.84 & 1.88 & 1.85 & 2.06 & & 1.60 & 1.70 & & & & & & & & & & & & & \\
\hline & 19 & Oncorhynchus HSC71 (AAB21658) & 1.75 & 1.86 & & & & 1.40 & 1.35 & & & & 1.52 & 1.56 & 1.64 & 1.90 & 2.53 & 1.51 & 1.49 & 1.54 & & & & & & & & & & & & \\
\hline & 20 & X. maculatus HSC70 (AB062115) & & & 2.63 & 2.34 & & 1.55 & 1.59 & & & & 2.51 & & & & & 1.97 & 1.65 & 2.63 & 1.25 & & & & & & & & & & & \\
\hline & 21 & Human_HSPA8 (NP_006588) & & & & & & & 3.36 & & & & 1.78 & 1.85 & 1.51 & 1.59 & 2.00 & 1.71 & 1.76 & 1.72 & 1.29 & & & & & & & & & & & \\
\hline & 22 & Rattus HSC71 (NM_024351) & & & 2.77 & & & 2.26 & 2.22 & & & & 1.81 & 1.82 & 1.80 & 2.36 & 2.48 & 1.84 & 1.93 & 1.88 & 1.25 & 1.80 & 0.52 & & & & & & & & & \\
\hline & 23 & Pelodiscus HSC70 (HQ219723) & & 3.28 & & & & & & & & 3.40 & & & 1.69 & 1.78 & 2.28 & 2.48 & 1.72 & & 1.58 & 2.07 & 1.11 & 1.23 & & & & & & & & \\
\hline & 24 & Bos HSP70 (U09861) & 1.55 & 1.48 & & & & 1.38 & 1.42 & & & & & & & & & & 2.17 & & 1.90 & 1.57 & & & & & & & & & & \\
\hline & 25 & Human_HSPA1A (NM_005345) & 1.46 & 1.42 & & & 2.48 & 1.28 & 1.31 & & & & & & & & & 2.47 & 1.65 & 2.56 & 1.47 & 1.27 & 2.86 & 2.27 & 2.45 & 0.22 & & & & & & \\
\hline & 26 & Rattus HSP70_1A (NM_031971) & 1.43 & 1.39 & & & & 1.10 & 1.09 & & & & & & & & & & 2.08 & & & 1.46 & & & & 0.35 & 0.35 & & & & & \\
\hline & 27 & X. maculatus HSP70-1 (AB062113) & & 2.43 & & & & & & & & & 3.59 & & 2.75 & 2.98 & & 2.32 & 1.87 & 2.12 & 2.98 & & & & 2.59 & 2.33 & 1.87 & 2.88 & & & & \\
\hline & 28 & X. maculatus HSP70-2 (AB062114) & 2.02 & 1.71 & & & 2.33 & 1.22 & 1.24 & 3.00 & & & & & & & & 1.85 & 1.65 & 2.32 & 1.37 & 1.27 & 2.30 & 1.81 & 2.40 & 0.86 & 0.73 & 1.21 & 1.37 & & & \\
\hline & 29 & Human_HSPA1L (NM_005527) & 2.64 & 2.50 & 2.64 & & & 1.87 & 1.92 & & & & & & 2.22 & & 3.46 & 2.18 & 1.76 & 2.21 & 1.44 & 1.66 & 1.67 & 1.43 & 2.14 & 0.96 & 0.81 & 1.25 & & 1.09 & & \\
\hline & 30 & Human_HSPA6 (NP_002146) & 1.62 & 1.44 & 1.86 & & & 1.55 & 1.57 & & & & & & 2.41 & 3.15 & & 2.20 & 1.57 & & 1.44 & 1.34 & 2.19 & 1.61 & 2.27 & 0.80 & 0.71 & 0.79 & 3.10 & 0.92 & 1.12 & \\
\hline & 31 & Alligator_HSP70 (AB306279) & 1.18 & 1.08 & 1.28 & & & 0.82 & 0.84 & & & & & & & & & 2.36 & 1.74 & & 2.01 & 1.29 & & & & 0.89 & 0.90 & 0.52 & 2.19 & 1.11 & $1 \quad 1.92$ & 1.04 \\
\hline
\end{tabular}

\begin{tabular}{|c|c|c|c|c|c|c|c|c|c|c|c|c|c|c|c|c|c|c|c|c|c|c|c|c|c|c|c|c|c|c|c|c|}
\hline \multirow{2}{*}{\multicolumn{2}{|c|}{ Gene No. }} & & \multicolumn{5}{|c|}{ Clade 1} & \multicolumn{3}{|c|}{ Clade 2} & \multicolumn{4}{|c|}{ Clade 3} & \multicolumn{6}{|c|}{ Clade 4} & \multicolumn{5}{|c|}{ Clade 5 (node 8) } & \multicolumn{7}{|c|}{ Clade 5 (node 9) } \\
\hline & & & 1 & 2 & 3 & 4 & 5 & 6 & 7 & 8 & 9 & 10 & 11 & 12 & 13 & 14 & 15 & 16 & 17 & 18 & 19 & 20 & 21 & 22 & 23 & 24 & 25 & 26 & 27 & 28 & 29 & 30 \\
\hline \multirow{4}{*}{$\begin{array}{l}\frac{d}{0} \\
\frac{\sigma}{0}\end{array}$} & 2 & D. melanogaster HSP70Aa (AF295933) & 0.04 & & & & & & & & & & & & & & & & & & & & & & & & & & & & & \\
\hline & 3 & Spodoptera HSP70 (FJ754276) & 0.06 & 0.06 & & & & & & & & & & & & & & & & & & & & & & & & & & & & \\
\hline & 4 & Bombyx HSP70 (АВ035326) & 0.07 & 0.07 & 0.03 & & & & & & & & & & & & & & & & & & & & & & & & & & & \\
\hline & 5 & Paralvinella HSP70-1 (EF580992) & & & 0.05 & & & & & & & & & & & & & & & & & & & & & & & & & & & \\
\hline \multirow{3}{*}{$\begin{array}{l}\frac{\tilde{D}}{0} \\
\frac{\pi}{U}\end{array}$} & 6 & B. plicatilis_HSP70-1 (AB775784) & 0.07 & 0.08 & 0.09 & 0.06 & & & & & & & & & & & & & & & & & & & & & & & & & & \\
\hline & 7 & B. plicatilis_HSP70-2 (AB775785) & 0.07 & 0.08 & 0.09 & 0.05 & & 0.13 & & & & & & & & & & & & & & & & & & & & & & & & \\
\hline & 8 & A. brightwelli_HSP70 (KX119429) & & & & 0.04 & 0.06 & 0.03 & 0.03 & & & & & & & & & & & & & & & & & & & & & & & \\
\hline \multirow{5}{*}{$\begin{array}{l}\frac{m}{0} \\
\frac{\pi}{u}\end{array}$} & 9 & Wuchereria (AF167352) & & & & & & & & & & & & & & & & & & & & & & & & & & & & & & \\
\hline & 10 & Setaria (AF079360) & & & & & & & & & 0.01 & & & & & & & & & & & & & & & & & & & & & \\
\hline & 11 & C. elegans HSP70A (M18540) & & & & & & & & & & & & & & & & & & & & & & & & & & & & & & \\
\hline & 12 & C. elegans HSP-1 (NM_070667) & & & & & & & & & & 0.01 & 0.53 & & & & & & & & & & & & & & & & & & & \\
\hline & 13 & M. nipponense HSP70-1 (KC460343) & & & & & & & & & 0.03 & 0.03 & 0.05 & 0.04 & & & & & & & & & & & & & & & & & & \\
\hline \multirow{5}{*}{$\begin{array}{l}\frac{\pi}{0} \\
\frac{\pi}{0}\end{array}$} & 14 & M. nipponense HSP7O-2 (DQ660140) & & & & & & & & & & & 0.04 & 0.03 & 0.03 & & & & & & & & & & & & & & & & & \\
\hline & 15 & Mytilus HSP70 (AY861684) & & & & & & & & & 0.04 & & & & 0.03 & 0.02 & & & & & & & & & & & & & & & & \\
\hline & 16 & Perinereis HSP70-1 (KU255783) & & & 0.07 & 0.05 & & 0.05 & 0.05 & & & & 0.07 & 0.06 & 0.02 & & 0.02 & & & & & & & & & & & & & & & \\
\hline & 17 & Paralvinella HSP70-2 (EF580993) & 0.09 & 0.09 & 0.08 & 0.06 & & 0.05 & 0.05 & & 0.03 & 0.04 & & & 0.05 & 0.05 & 0.03 & 0.04 & & & & & & & & & & & & & & \\
\hline & 18 & Haliotis HSP7Oc (FJ812176) & & & & & & & & & & & 0.06 & 0.06 & 0.04 & 0.04 & & 0.04 & 0.04 & & & & & & & & & & & & & \\
\hline \multirow{6}{*}{ 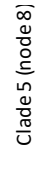 } & 19 & Oncorhynchus HSC71 (AAB21658) & 0.09 & 0.09 & & & & 0.06 & 0.06 & & & & 0.07 & 0.06 & 0.04 & 0.03 & 0.03 & 0.04 & 0.05 & 0.05 & & & & & & & & & & & & \\
\hline & 20 & X. maculatus HSC70 (AB062115) & & & 0.06 & 0.07 & & 0.06 & 0.06 & & & & 0.05 & & & & & 0.04 & 0.05 & 0.03 & 0.03 & & & & & & & & & & & \\
\hline & 21 & Human_HSPA8 (NP_006588) & & & & & & & 0.02 & & & & 0.06 & 0.05 & 0.04 & 0.04 & 0.03 & 0.04 & 0.04 & 0.04 & 0.02 & & & & & & & & & & & \\
\hline & 22 & Rattus HSC71 (NM_024351) & & & 0.05 & & & 0.03 & 0.03 & & & & 0.06 & 0.05 & 0.04 & 0.03 & 0.02 & 0.04 & 0.04 & 0.03 & 0.02 & 0.03 & 0.00 & & & & & & & & & \\
\hline & 23 & Pelodiscus HSC70 (HQ219723) & & 0.05 & & & & & & & & 0.02 & & & 0.04 & 0.03 & 0.03 & 0.03 & 0.04 & & 0.02 & 0.02 & 0.00 & 0.00 & & & & & & & & \\
\hline & 24 & Bos HSP70 (U09861) & 0.10 & 0.10 & & & & 0.07 & 0.07 & & & & & & & & & & 0.05 & & 0.04 & 0.06 & & & & & & & & & & \\
\hline \multirow{7}{*}{ 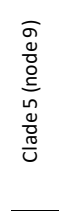 } & 25 & Human_HSPA1A (NM_005345) & 0.10 & 0.10 & & & 0.06 & 0.07 & 0.07 & & & & & & & & & 0.04 & 0.07 & 0.04 & 0.05 & 0.07 & 0.02 & 0.03 & 0.03 & 0.02 & & & & & & \\
\hline & 26 & Rattus HSP70_1A (NM_031971) & 0.11 & 0.11 & & & & 0.09 & 0.09 & & & & & & & & & & 0.05 & & & 0.06 & & & & 0.04 & 0.04 & & & & & \\
\hline & 27 & X. maculatus HSP70-1 (AB062113) & & 0.07 & & & & & & & & & 0.03 & & 0.03 & 0.03 & & 0.04 & 0.05 & 0.05 & 0.02 & & & & 0.02 & 0.03 & 0.04 & 0.02 & & & & \\
\hline & 28 & X. maculatus HSP70-2 (AB062114) & 0.08 & 0.09 & & & 0.07 & 0.08 & 0.08 & 0.03 & & & & & & & & 0.05 & 0.07 & 0.05 & 0.06 & 0.07 & 0.03 & 0.04 & 0.03 & 0.08 & 0.09 & 0.06 & 0.04 & & & \\
\hline & 29 & Human_HSPA1L (NM_005527) & 0.06 & 0.06 & 0.06 & & & 0.05 & 0.05 & & & & & & 0.04 & & 0.03 & 0.04 & 0.06 & 0.05 & 0.06 & 0.06 & 0.05 & 0.06 & 0.04 & 0.04 & 0.05 & 0.03 & & 0.06 & & \\
\hline & 30 & Human_HSPA6 (NP_002146) & 0.10 & 0.11 & 0.09 & & & 0.08 & 0.07 & & & & & & 0.05 & 0.04 & & 0.05 & 0.08 & & 0.08 & 0.09 & 0.05 & 0.06 & 0.05 & 0.09 & 0.10 & 0.09 & 0.04 & 0.12 & 0.08 & \\
\hline & 31 & Alligator_HSP70 (AB306279) & 0.13 & 0.15 & 0.13 & & & 0.11 & 0.11 & & & & & & & & & 0.04 & 0.06 & & 0.04 & 0.07 & & & & 0.10 & 0.10 & 0.16 & 0.04 & 0.08 & 0.05 & 0.11 \\
\hline
\end{tabular}


Supplementary material 2

Heat Treatment

For semi-quantitative RT-PCR, rotifers were batch-cultured in $400 \mathrm{~mL}$ at $25^{\circ} \mathrm{C}$. In the exponential growth phase, approximately $6 \times 10^{4}$ individuals were randomly divided into two groups and concentrated to about $10 \mathrm{~mL}$ by filtration through $20 \mu \mathrm{m}$ mesh filters. One group (heat-treated group) was transferred to culture medium at $30^{\circ} \mathrm{C}$ for $10 \mathrm{~min}$, collected with a $20 \mu \mathrm{m}$ mesh filter, and stored in ISOGEN (Nippon Gene, Tokyo, Japan) at $-80^{\circ} \mathrm{C}$. The control group was prepared by the same procedure at $25^{\circ} \mathrm{C}$.

For quantitative real-time PCR, 8 rotifers bearing 2 or 3 eggs were selected from a batch culture population and inoculated into a well of a 12-well plate (Nunc, Rochester, NY) containing $1 \mathrm{~mL}$ of culture medium at $25^{\circ} \mathrm{C}$. The 12 -well plate was put on a water bath at $40^{\circ} \mathrm{C}$ for $10 \mathrm{~min}$, and then transferred to a water bath at $25^{\circ} \mathrm{C}$. Rotifers were sampled before heat treatment (control), just after heat treatment (time 0$)$, and 2, 3, 4, 6, and $8 \mathrm{~h}$ after heat treatment ( $\mathrm{n}=3$, each group contained 8 individuals). Rotifer samples were stored in $500 \mu \mathrm{L}$ ISOGEN (Nippon Gene) and stored at $-80^{\circ} \mathrm{C}$ until use. Rotifers used for in situ hybridization were prepared by the same method except the heat shock temperature was $30^{\circ} \mathrm{C}$.

cDNA Cloning of HSP70

5' RACE for the partial sequence of B. plicatilis HSP70 gene [AB076052; (Kaneko et al. 2002)] was carried out using a GeneRacer kit (Invitrogen, Carlsbad, CA) with gene-specific primers rHSP70_5RACE1, rHSP70_5RACE2, and rHSP70_5RACE3 (supplementary table S1). Total RNA was extracted from a rotifer population in an exponential growth phase and used for 1st strand cDNA synthesis using the rHSP70_5RACE1 primer and the GeneRacer kit (Invitrogen). First round PCR was performed at $94^{\circ} \mathrm{C}$ for $3 \mathrm{~min}$ followed by 30 cycles of $94^{\circ} \mathrm{C}$ for $30 \mathrm{~s}, 55^{\circ} \mathrm{C}$ for $30 \mathrm{~s}$ and $72{ }^{\circ} \mathrm{C}$ for 1 min. The final extension step was performed at $72^{\circ} \mathrm{C}$ for $5 \mathrm{~min}$. The $20 \mu \mathrm{L}$-reaction mixture contained about 1 $\mu \mathrm{g}$ of the 1st strand cDNA, $2 \mu \mathrm{L}$ of Ex Taq buffer (Takara, Shiga, Japan), 1 U of Ex Taq DNA polymerase, $4 \mathrm{nmol}$ of dNTP mixture, 10 pmol of rHSP70_5RACE2 primer and GeneRacer 5' primer (Table 1). The amplified products were diluted 10-fold with sterile distilled water and used as the template for the nested PCR with rHSP70_5RACE3 and GeneRacer 5' nested primers (supplementary table S1). The PCR conditions were same as the first round PCR except for the template and primers. The amplified products were subcloned into pGEM-T vector (Promega, Madison, WI) and sequenced as reported previously (Yoon et al. 2008).

3' RACE for rotifer HSP70 gene was carried out as follows. An aliquot of first strand cDNA was synthesized from rotifers in an exponential phase using an oligo-dT adapter primer (supplementary table $\mathrm{S} 1)$. PCR was performed with the first strand cDNA as a template and with rHSP70_3RACE1 and AUAP primers. PCR was carried out with 30 cycles of $94^{\circ} \mathrm{C}$ for $30 \mathrm{~s}, 60^{\circ} \mathrm{C}$ for $30 \mathrm{~s}$, and $72^{\circ} \mathrm{C}$ for $2 \mathrm{~min}$. The final extension step was performed at $72^{\circ} \mathrm{C}$ for $5 \mathrm{~min}$. The $20 \mu \mathrm{l}$ of reaction mixture contained 5 pmol of forward and reverse primers, approximately $1 \mu \mathrm{g}$ of first strand cDNA, $4 \mathrm{nmol}$ of dNTP mixture, $2 \mu \mathrm{L}$ of $10 \times$ PCR buffer [100 mM Tris-HCl, pH8.3, $500 \mathrm{mM} \mathrm{KCl,} 15 \mathrm{mM} \mathrm{MgCl}_{2}, 0.01 \%$ (w/v) gelatin] and $0.2 \mathrm{U}$ of Ex Taq DNA polymerase (Takara). Nested PCR was performed under the same conditions using the PCR product diluted 100-fold and the rHSP70_3RACE2 primer. The amplified products were subcloned into pGEM-T vecter (Promega) and sequenced as described above. The nucleotide sequence of the open reading frame was confirmed by a single PCR using rHSP70_full_fl and rHSP70_full_r1 primers 
(supplementary table S1). Conditions of the single PCR were same as described in 5' RACE.

\section{Semi-Quantitative RT-PCR}

RNA extraction and 1st strand cDNA synthesis were performed as described above. Primers rHSP70_gapF and rHSP70_gapR were designed to amplify the region encoding the GGMP repeat of $B$. plicatilis HSP70cB1i and HSP70cB2i genes (supplementary table S1). The $\beta$-actin gene was used as the internal control with specific primers rActinF and rActinR reported previously (Kaneko et al. 2005). PCR was carried out at $94^{\circ} \mathrm{C}$ for $3 \mathrm{~min}$ followed by $20-40$ cycles of $94^{\circ} \mathrm{C}$ for $30 \mathrm{~s}, 58^{\circ} \mathrm{C}$ for $30 \mathrm{~s}$ and $72^{\circ} \mathrm{C}$ for $30 \mathrm{~s}$. A $20-\mu \mathrm{L}$ reaction mixture contained approximately $1 \mu \mathrm{g}$ of $1 \mathrm{st}$ strand cDNA, 10 pmol of rHSP70_gapF and rHSP70_gapR, $2 \mu \mathrm{L}$ of Ampli Taq buffer (Applied Biosystems, Foster City, CA), 4 nmol of dNTP mixture, and 0.2 U of Ampli Taq DNA polymerase (Applied Biosystems). The PCR products were separated with an $8 \%$ acrylamide gel and visualized by ethidium bromide staining.

Quantitative Real-Time PCR

Rotifers were collected $0,2,4,6$, and $8 \mathrm{~h}$ after the heat treatment, and stored in ISOGEN (Takara) at $-80^{\circ} \mathrm{C}$ until use (three replicates for each time point, one replicate contained eight individuals). Rotifers collected before the heat treatment were used as the control. Quantitative real-time PCR was performed as reported previously (Kaneko et al. 2011). Primers for the $\beta$-actin gene were described previously (Kaneko et al. 2005); primers for HSP70 genes were designed using the Primer Express software ver. 2 (Applied Biosystems) (supplementary table S1). R version 3.5.1. and the multcomp package (Hothorn et al. 2008) was used for one-way analysis of variance (ANOVA) followed by the Dunnett's test on the Macintosh platform.

In situ Hybridization

Rotifers were fixed in PBS containing 4\% paraformaldehyde $4 \mathrm{~h}$ after the heat treatment. In situ hybridization was performed as reported previously (Kaneko et al. 2011), using DIG-labeled RNA probes synthesized as follows. Primers HSP70_insituF and HSP70_insituR were designed to amplify the DNA fragment of 2026 - 2103 nt of B. plicatilis HSP70cB1i gene (supplementary table S1). PCR was performed in a $20 \mu \mathrm{L}$ reaction mixture containing approximately $1 \mu \mathrm{g}$ of the genomic DNA of $B$. plicatilis, 10 pmol each of the HSP70_insituF and HSP70_insituR primers, $2 \mu \mathrm{L}$ of $10 \times$ PCR buffer, 4 nmol dNTP mixture, and $0.2 \mathrm{U}$ of Ex Taq DNA polymerase (Takara). The amplification was carried out with the initial denaturation at $94^{\circ} \mathrm{C}$ for $3 \mathrm{~min}$ and 40 cycles of $94^{\circ} \mathrm{C}$ for $30 \mathrm{~s}, 60^{\circ} \mathrm{C}$ for $30 \mathrm{~s}$, and $72^{\circ} \mathrm{C}$ for $1.5 \mathrm{~min}$, followed by the final extension step at $72^{\circ} \mathrm{C}$ for $5 \mathrm{~min}$. The amplified products were subcloned into pGEM-T easy vector (Promega). DIG-labeled RNA probes were synthesized with a DIG RNA labeling kit (Roche) according to the manufacturer's instructions.

Hothorn T, Bretz F, Westfall P. 2008. Simultaneous inference in general parametric models. Biom J 50:346363.

Kaneko G, Kinoshita S, Yoshinaga T, Tsukamoto K, Watabe S. 2002. Changes in expression patterns of stress protein genes during population growth of the rotifer Brachionus plicatilis. Fish Sci 68:1317-1323. Kaneko G, Yoshinaga T, Yanagawa Y, Kinoshita S, Tsukamoto K, Watabe S. 2005. Molecular characterization of Mn-superoxide dismutase and gene expression studies in dietary restricted Brachionus plicatilis rotifers. Hydrobiologia 546:117-123.

Kaneko G, Yoshinaga T, Yanagawa Y, Ozaki Y, Tsukamoto K, Watabe S. 2011. Calorie restriction-induced 
bioRxiv preprint doi: https://doi.org/10.1101/2020.09.21.307264; this version posted April 22, 2021. The copyright holder for this preprint (which

was not certified by peer review) is the author/funder, who has granted bioRxiv a license to display the preprint in perpetuity. It is made available under aCC-BY-NC-ND 4.0 International license.

maternal longevity is transmitted to their daughters in a rotifer. Funct Ecol 25:209-216.

Yoon SH, Itoh Y, Kaneko G, Nakaniwa M, Ohta M, Watabe S. 2008. Molecular characterization of Japanese sillago vitellogenin and changes in its expression levels on exposure to 17 beta-estradiol and 4-tertoctylphenol. Mar Biotechnol 10:19-30. 